\title{
Enantioselective synthesis of furo[2,3-b]furans, a spongiane diterpenoid substructure
}

\section{Supporting Information}

Roland Weisser, Weimin Yue, and Oliver Reiser*

Institut für Organische Chemie der Universität Regensburg, Universitätsstr. 31, D-93053 Regensburg, Germany

Oliver.Reiser@chemie.uni-regensburg.de

(1S,5S,6S)-6-ethyl 3-methyl 2-oxabicyclo[3.1.0]hex-3-ene-3,6-dicarboxylate (9)

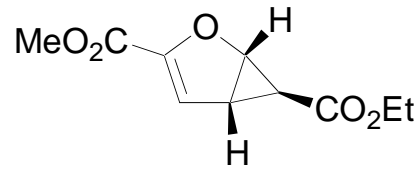

Fp: $42{ }^{\circ} \mathrm{C}$.

${ }^{1} \mathbf{H}-\mathbf{N M R}\left(300 \mathrm{MHz}, \mathrm{CDCl}_{3}\right): \delta(\mathrm{ppm})=6.32\left(\mathrm{~d},{ }^{3} J=2.8 \mathrm{~Hz}, 1 \mathrm{H}, \mathrm{H}-4\right), 4.90\left(\mathrm{dd},{ }^{3} J=5.4 \mathrm{~Hz}\right.$, $\left.{ }^{3} J=1.1 \mathrm{~Hz}, 1 \mathrm{H}, \mathrm{H}-1\right), 4.08\left(\mathrm{q},{ }^{3} J=7.1 \mathrm{~Hz}, 2 \mathrm{H}, \mathrm{CH}_{2}\right), 3.74\left(\mathrm{~s}, 3 \mathrm{H},-\mathrm{OCH}_{3}\right), 2.80$ (ddd, ${ }^{3} J=$ $\left.5.4 \mathrm{~Hz},{ }^{3} J=2.8 \mathrm{~Hz},{ }^{3} J=2.7 \mathrm{~Hz}, 1 \mathrm{H}, \mathrm{H}-5\right), 1.20\left(\mathrm{t},{ }^{3} J=7.1 \mathrm{~Hz}, 3 \mathrm{H}, \mathrm{CH}_{2} \mathrm{CH}_{3}\right), 1.09$ (dd, ${ }^{3} J=$ $\left.2.7 \mathrm{~Hz},{ }^{3} \mathrm{~J}=1.1 \mathrm{~Hz}, 1 \mathrm{H}, \mathrm{H}-6\right) .{ }^{13} \mathrm{C}-\mathrm{NMR}\left(75 \mathrm{MHz}, \mathrm{CDCl}_{3}\right): \delta(\mathrm{ppm})=171.77\left(\mathrm{C}_{\mathrm{q}}, \underline{\mathrm{CO}_{2} \mathrm{Et}}\right)$,

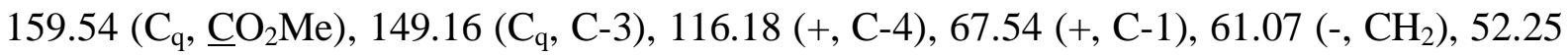
$\left(+, \mathrm{OCH}_{3}\right), 31.97(+, \mathrm{C}-5), 21.46(+, \mathrm{C}-6), 14.20\left(+, \mathrm{CH}_{2} \underline{\mathrm{CH}}_{3}\right) . \mathbf{I R}(\mathrm{KBr}): v\left(\mathrm{~cm}^{-1}\right)=3139$, 3067, 2992, 2958, 2908, 2851, 1715, 1625, 1439, 1399, 1382, 1343, 1301, 1217, 1183, 1113 , 1085, 1040, 1009, 988, 970, 932, 878, 831, 795, 733. $[\alpha]_{\mathbf{D}}{ }^{\mathbf{2 0}}\left(\mathrm{CH}_{2} \mathrm{Cl}_{2}, \mathrm{c}=1.0\right)=-272 . \mathbf{M S}$ $\left(\right.$ EI-MS): $m / z(\%)=212.3(10)\left[\mathrm{M}^{+}\right], 153.4$ (12), $\left[\mathrm{M}^{+} \Delta \mathrm{CO}_{2} \mathrm{Me}\right], 139.4$ (100) $\left[\mathrm{M}^{+} \Delta \mathrm{CO}_{2} \mathrm{Et}\right]$, 125.4 (19), 97.3 (23). $\mathrm{C}_{10} \mathrm{H}_{12} \mathrm{O}_{5}$ (212.2): calcd. C 56.60, H 5.70; found C 56.51, H 5.73.

(1S,3R,5S,6S)-6-ethyl 3-methyl 2-oxabicyclo[3.1.0]hexane-3,6-dicarboxylate (10)

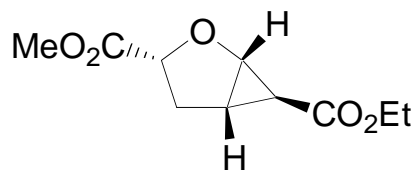

Fp: $69{ }^{\circ} \mathrm{C}$. 
${ }^{1}$ H-NMR $\left(300 \mathrm{MHz}, \mathrm{CDCl}_{3}\right): \delta(\mathrm{ppm})=4.74\left(\mathrm{dd},{ }^{3} J=10.7 \mathrm{~Hz},{ }^{3} J=4.3 \mathrm{~Hz}, 1 \mathrm{H}, \mathrm{H}-3\right), 4.28$ $\left(\mathrm{dd},{ }^{3} J=5.7 \mathrm{~Hz},{ }^{3} J=0.9 \mathrm{~Hz}, 1 \mathrm{H}, \mathrm{H}-1\right), 4.06\left(\mathrm{dq},{ }^{2} J=10.8 \mathrm{~Hz},{ }^{3} J=7.1 \mathrm{~Hz}, 1 \mathrm{H}, \mathrm{CHHCH}_{3}\right.$ ), $4.02\left(\mathrm{dq},{ }^{2} J=10.8 \mathrm{~Hz},{ }^{3} J=7.1 \mathrm{~Hz}, 1 \mathrm{H}, \mathrm{CHHCH}_{3}\right), 3.71\left(\mathrm{~s}, 3 \mathrm{H}, \mathrm{OCH}_{3}\right), 2.60\left(\mathrm{ddd},{ }^{2} J=13.4\right.$ $\left.\mathrm{Hz},{ }^{3} J=10.7 \mathrm{~Hz},{ }^{3} J=6.0 \mathrm{~Hz}, 1 \mathrm{H}, \mathrm{H}-4_{\text {exo }}\right), 2.23\left(\mathrm{dd},{ }^{2} J=13.4 \mathrm{~Hz},{ }^{3} J=4.3 \mathrm{~Hz}, 1 \mathrm{H}, \mathrm{H}-4_{\text {endo }}\right.$ ), $2.13\left(\mathrm{ddd},{ }^{3} J=6.0 \mathrm{~Hz},{ }^{3} J=5.7 \mathrm{~Hz},{ }^{3} J=4.0 \mathrm{~Hz}, 1 \mathrm{H}, \mathrm{H}-5\right), 1.83\left(\mathrm{dd},{ }^{3} J=4.0 \mathrm{~Hz},{ }^{3} J=0.9 \mathrm{~Hz}\right.$, $1 \mathrm{H}, \mathrm{H}-6), 1.18\left(\mathrm{dd},{ }^{3} J=7.1 \mathrm{~Hz},{ }^{3} J=7.1 \mathrm{~Hz}, 3 \mathrm{H}, \mathrm{CH}_{2} \mathrm{CH}_{3}\right) .{ }^{13} \mathbf{C}-\mathbf{N M R}\left(75 \mathrm{MHz}, \mathrm{CDCl}_{3}\right): \delta$ $(\mathrm{ppm})=173.33\left(\mathrm{C}_{\mathrm{q}}, \mathrm{C}=\mathrm{O}\right), 170.40\left(\mathrm{C}_{\mathrm{q}}, \mathrm{C}=\mathrm{O}\right), 79.11(+, \mathrm{C}-3), 67.46(+, \mathrm{C}-1), 60.55(-$, $\left.\underline{\mathrm{CH}}_{2} \mathrm{CH}_{3}\right), 52.48\left(+, \mathrm{OCH}_{3}\right), 32.02\left(-, \mathrm{CH}_{2} \mathrm{CH}\right), 28.20(+, \mathrm{C}-6), 25.58(+, \mathrm{C}-5), 14.22(+$, $\left.\mathrm{CH}_{2} \mathrm{CH}_{3}\right)$. IR $(\mathrm{KBr}): v\left(\mathrm{~cm}^{-1}\right)=3070,2986,2943,2905,1744,1713,1457,1435,1416,1357$, 1328, 1298, 1279, 1211, 1196, 1171, 1115, 1084, 1048, 1029, 981, 963, 929, 905, 878, 845, 809, 737, 718, 702, 674, 653, 474, 417. $[\alpha]_{\mathbf{D}}{ }^{20}\left(\mathrm{CH}_{2} \mathrm{Cl}_{2}, \mathrm{c}=1.4\right)=+27 . \mathbf{M S}(\mathrm{EI}-\mathrm{MS}): \mathrm{m} / \mathrm{z}$ $(\%)=214.1$ (4) $\left[\mathrm{M}^{+}\right], 169.1 \quad(17) \quad\left[\mathrm{M}^{+} \Delta \mathrm{OEt}\right], 168.1 \quad$ (41) $\left[\mathrm{M}^{+} \Delta \mathrm{EtOH}\right], 155.1 \quad$ (25) $\left[\mathrm{M}^{+} \Delta \mathrm{CO}_{2} \mathrm{Me}\right], 141.1$ (28) [ $\left[\mathrm{M}^{+} \Delta \mathrm{CO}_{2} \mathrm{Et}\right], 136.1$ (38) , 113.1 (49), 109.1 (56), 81.1 (100), 55.1 (39), 53.1 (39), 29.2 (43) [ $\left[\mathrm{tt}^{+}\right]$. HRMS (EI-MS): für $\mathrm{C}_{10} \mathrm{H}_{14} \mathrm{O}_{5}\left(\mathrm{M}^{+}\right)$calcd. 214.0841, found 214.0841.

$(2 R, 3 \mathrm{a} S, 6 \mathrm{a} R)-5-0 x o h e x a h y d r o f u r o[2,3-b]$ furan-2-carboxylic acid (11)

Fp: $103{ }^{\circ} \mathrm{C}$.

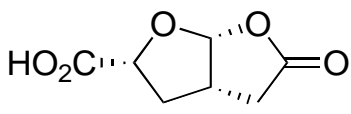

${ }^{1}$ H-NMR $\left(400 \mathrm{MHz}\right.$, DMSO- $\left.d_{6}\right): \delta(\mathrm{ppm})=12.89\left(\mathrm{~s}, \mathrm{br}, 1 \mathrm{H}, \mathrm{CO}_{2} \mathrm{H}\right), 6.07\left(\mathrm{~d},{ }^{3} \mathrm{~J}=5.7 \mathrm{~Hz}, 1\right.$ H, H-6a), 4.66 (dd, ${ }^{3} J=8.9 \mathrm{~Hz},{ }^{3} J=4.2 \mathrm{~Hz}, 1 \mathrm{H}, \mathrm{H}-2$ ), 3.17 (dddd, ${ }^{3} J=10.1 \mathrm{~Hz},{ }^{3} J=9.6$ $\left.\mathrm{Hz},{ }^{3} J=5.7 \mathrm{~Hz},{ }^{3} J=4.3 \mathrm{~Hz},{ }^{3} J=3.4 \mathrm{~Hz}, 1 \mathrm{H}, \mathrm{H}-3 \mathrm{a}\right), 2.82\left(\mathrm{dd},{ }^{2} J=18.3 \mathrm{~Hz},{ }^{3} J=10.1 \mathrm{~Hz}, 1\right.$ H, H-4 exo ), $2.46\left(\mathrm{ddd},{ }^{2} J=13.3 \mathrm{~Hz},{ }^{3} J=9.6 \mathrm{~Hz},{ }^{3} J=8.9 \mathrm{~Hz}, 1 \mathrm{H}, \mathrm{H}-3_{\text {exo }}\right.$ ), $2.35\left(\mathrm{dd},{ }^{2} J=18.3\right.$ $\mathrm{Hz},{ }^{3} J=3.4 \mathrm{~Hz}, 1 \mathrm{H}, \mathrm{H}-4_{\text {endo }}$ ), 1.98 ( $\left(\mathrm{ddd},{ }^{2} J=13.3 \mathrm{~Hz},{ }^{3} J=4.3 \mathrm{~Hz},{ }^{3} J=4.2 \mathrm{~Hz}, 1 \mathrm{H}, \mathrm{H}-3_{\text {endo }}\right.$ ). ${ }^{13}$ C-NMR $\left(100 \mathrm{MHz}\right.$, DMSO- $\left.d_{6}\right): \quad \delta(\mathrm{ppm})=175.04\left(\mathrm{C}_{\mathrm{q}}, \mathrm{C}-5\right), 172.87\left(\mathrm{C}_{\mathrm{q}}, \mathrm{CO}_{2} \mathrm{H}\right), 108.65$ (+, C-6a), $78.32\left(+\right.$, C-2), 37.93 (+, C-3a), 34.68 (-, C-4), $33.95\left(-\right.$, C-3). IR $(\mathrm{KBr}): v\left(\mathrm{~cm}^{-1}\right)=$ 3436, 2983, 2955, 1762, 1731, 1715, 1462, 1421, 1414, 1394, 1356, 1328, 1307, 1292, 1265 , $1240,1220,1186,1100,1060,1011,970,928,880,870,829,778,693,668 .[\alpha]_{\mathbf{D}}{ }^{20}(\mathrm{MeOH}$, $\mathrm{c}=1.9)=-77$. MS (CI-MS): $m / z(\%)=190.0(100)\left[\mathrm{M}+\mathrm{NH}_{4}{ }^{+}\right]$. HRMS (EI-MS): für $\mathrm{C}_{7} \mathrm{H}_{8} \mathrm{O}_{5}$ $\left(\mathrm{M}^{+}\right)$calcd. 172.0372 , found $172.0376 . \mathbf{C}_{7} \mathbf{H}_{8} \mathbf{O}_{5}$ : calcd. C 48.84, H 4.68, found $\mathrm{C} 48.76, \mathrm{H}$ 4.79 . 
(2S,3aS,6aR)-5-oxohexahydrofuro[2,3-b]furan-2-yl acetate (13)

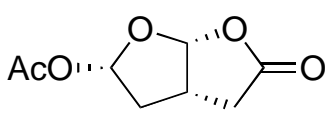

Fp: $65^{\circ} \mathrm{C}$.

${ }^{1}$ H-NMR $\left(300 \mathrm{MHz}, \mathrm{CDCl}_{3}\right): \delta(\mathrm{ppm})=6.45\left(\mathrm{~d},{ }^{3} \mathrm{~J}=4.9 \mathrm{~Hz}, 1 \mathrm{H}, \mathrm{H}-2\right), 6.19\left(\mathrm{~d},{ }^{3} J=6.1 \mathrm{~Hz}\right.$, $1 \mathrm{H}, \mathrm{H}-6 \mathrm{a}$ ), 3.27 (ddddd, ${ }^{3} J=11.2 \mathrm{~Hz},{ }^{3} J=9.6 \mathrm{~Hz},{ }^{3} J=6.1 \mathrm{~Hz},{ }^{3} J=4.2 \mathrm{~Hz},{ }^{3} J=1.2 \mathrm{~Hz}, \mathrm{H}-$ 3a), $2.91\left(\mathrm{dd},{ }^{2} J=18.6 \mathrm{~Hz},{ }^{3} J=11.2 \mathrm{~Hz}, 1 \mathrm{H}, \mathrm{H}-4_{\text {exo }}\right), 2.62\left(\mathrm{dd},{ }^{2} J=18.6 \mathrm{~Hz},{ }^{3} J=4.2 \mathrm{~Hz}, 1\right.$ H, H-4 endo $_{\text {) }} 2.40$ (ddd, $\left.{ }^{2} J=14.0 \mathrm{~Hz},{ }^{3} J=9.6 \mathrm{~Hz},{ }^{3} J=4.9 \mathrm{~Hz}, 1 \mathrm{H}, \mathrm{H}-3_{\text {exo }}\right), 2.07\left(\mathrm{dd},{ }^{2} J=14.0\right.$ $\left.\mathrm{Hz},{ }^{3} J=1.2 \mathrm{~Hz}, 1 \mathrm{H}, \mathrm{H}-3_{\text {endo }}\right), 2.05\left(\mathrm{~s}, 3 \mathrm{H}, \mathrm{CH}_{3}\right) .{ }^{13} \mathbf{C}-\mathbf{N M R}\left(75 \mathrm{MHz}, \mathrm{CDCl}_{3}\right): \delta(\mathrm{ppm})=$ $174.04\left(\mathrm{C}_{\mathrm{q}}, \mathrm{C}-5\right), 168.52\left(\mathrm{C}_{\mathrm{q}}, \underline{\mathrm{COCH}}_{3}\right), 107.41$ (+, C-6a), 97.42 (+, C-2), 37.27 (-, C-3), 35.18 (+, C-3a), $34.47(-, \mathrm{C}-4), 20.20\left(+, \mathrm{CH}_{3}\right) . \mathbf{I R}(\mathrm{KBr}): v\left(\mathrm{~cm}^{-1}\right)=2985,2959,2928,1783,1722$, 1381, 1359, 1260, 1242, 1179, 1112, 1067, 1024, 975, 933, 919, 866, 833, 792, 773, 685. $[\boldsymbol{\alpha}]_{\mathbf{D}}{ }^{20}\left(\mathrm{CH}_{2} \mathrm{Cl}_{2}, \mathrm{c}=1.1\right)=-152 . \mathbf{M S}(\mathrm{CI}-\mathrm{MS}): m / z(\%)=204.0(100)\left[\mathrm{M}+\mathrm{NH}_{4}{ }^{+}\right] . \mathbf{C}_{\mathbf{8}} \mathbf{H}_{10} \mathbf{O}_{5}$ : calcd. C 51.61, H 5.41, found C 51.76, H 5.60.

(2R,3aS,6aR)-5-oxohexahydrofuro[2,3-b]furan-2-yl acetate (epi-13)

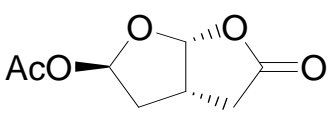

Fp: $=116-118^{\circ} \mathrm{C}$.

${ }^{1} \mathbf{H}-\mathbf{N M R}\left(400 \mathrm{MHz}, \mathrm{CDCl}_{3}\right): \delta(\mathrm{ppm})=6.49\left(\mathrm{ddd},{ }^{3} J=5.2 \mathrm{~Hz},{ }^{3} J=1.1 \mathrm{~Hz},{ }^{4} J=0.5 \mathrm{~Hz}, 1 \mathrm{H}\right.$, $\mathrm{H}-2), 6.10\left(\mathrm{~d},{ }^{3} J=5.5 \mathrm{~Hz}, 1 \mathrm{H}, \mathrm{H}-6 \mathrm{a}\right), 3.25$ (dddddd, ${ }^{3} J=9.4 \mathrm{~Hz},{ }^{3} J=8.7 \mathrm{~Hz},{ }^{3} J=8.7 \mathrm{~Hz},{ }^{3} J$ $\left.=5.5 \mathrm{~Hz},{ }^{3} \mathrm{~J}=1.9 \mathrm{~Hz},{ }^{4} \mathrm{~J}=0.5 \mathrm{~Hz}, 1 \mathrm{H}, \mathrm{H}-3 \mathrm{a}\right), 2.86\left(\mathrm{dd},{ }^{2} J=18.1 \mathrm{~Hz},{ }^{3} J=8.7 \mathrm{~Hz}, 1 \mathrm{H}, \mathrm{H}-\right.$ $\left.4_{\text {exo }}\right), 2.52\left(\mathrm{dd},{ }^{2} J=18.1 \mathrm{~Hz},{ }^{3} J=1.9 \mathrm{~Hz}, 1 \mathrm{H}, \mathrm{H}-4_{\text {endo }}\right), 2.40\left(\mathrm{ddd},{ }^{2} J=13.9 \mathrm{~Hz},{ }^{3} J=8.7 \mathrm{~Hz}\right.$, $\left.{ }^{3} J=1.1 \mathrm{~Hz}, 1 \mathrm{H}, \mathrm{H}-3_{\mathrm{exo}}\right), 2.07\left(\mathrm{~s}, 3 \mathrm{H}, \mathrm{CH}_{3}\right), 1.97$ (ddd, ${ }^{2} J=13.9 \mathrm{~Hz},{ }^{3} J=9.4 \mathrm{~Hz},{ }^{3} J=5.2 \mathrm{~Hz}$, $\left.1 \mathrm{H}, \mathrm{H}-3_{\text {endo }}\right) .{ }^{13} \mathbf{C}-\mathbf{N M R}\left(75 \mathrm{MHz}, \mathrm{CDCl}_{3}\right): \delta(\mathrm{ppm})=173.56\left(\mathrm{C}_{\mathrm{q}}, \mathrm{C}-5\right), 169.57\left(\mathrm{C}_{\mathrm{q}}, \underline{\mathrm{COCH}}_{3}\right)$, 107.59 (+, C-6a), 98.76 (+, C-2), 36.81 (-, C-3), 36.71 (+, C-3a), 34.78 (-, C-4), 21.08 (+, $\left.\mathrm{CH}_{3}\right)$. IR $(\mathrm{KBr}): v\left(\mathrm{~cm}^{-1}\right)=2975,1786,1739,1443,1420,1374,1355,1332,1296,1241$, 1219, 1184, 1165, 1103, 1088, 1048, 1009, 974, 952, 928, 860, 829, 780, 660, 642, 605. $[\alpha]_{\mathbf{D}}{ }^{20}\left(\mathrm{CH}_{2} \mathrm{Cl}_{2}, \mathrm{c}=0.9\right)=+18 . \mathbf{M S}(\mathrm{CI}-\mathrm{MS}): \mathrm{m} / z(\%)=127.0(66)\left[\mathrm{M}^{+} \Delta \mathrm{OAc}\right], 109.0(15)$, 99.0 (18) $\left[\mathrm{M}^{+} \Delta \mathrm{OAc} \Delta \mathrm{CO}\right], 82.0$ (28), 70.0 (23), 43.0 (100) $\left[\mathrm{CH}_{3} \mathrm{CO}^{+}\right], 42.0$ (17), 41.0 (12). $\mathbf{C}_{8} \mathbf{H}_{10} \mathbf{O}_{5}$ : calcd. C 51.61, H 5.41, found C 51.35, H 5.34. 

boxylate (14)

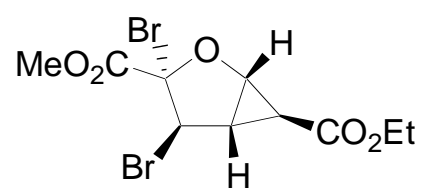

Fp: $97^{\circ} \mathrm{C}$.

${ }^{1}$ H-NMR $\left(300 \mathrm{MHz}, \mathrm{CDCl}_{3}\right): \delta(\mathrm{ppm})=5.11(\mathrm{~s}, 1 \mathrm{H}, \mathrm{H}-4), 4.89\left(\mathrm{dd},{ }^{3} J=5.3 \mathrm{~Hz},{ }^{3} J=1.1 \mathrm{~Hz}\right.$, $1 \mathrm{H}, \mathrm{H}-1), 4.15\left(\mathrm{q},{ }^{3} J=7.1 \mathrm{~Hz}, 2 \mathrm{H}, \mathrm{CH}_{2}\right), 3.88\left(\mathrm{~s}, 3 \mathrm{H}, \mathrm{OCH}_{3}\right), 3.66\left(\mathrm{dd},{ }^{3} J=4.0 \mathrm{~Hz},{ }^{3} J=1.1\right.$ $\mathrm{Hz}, 1 \mathrm{H}, \mathrm{H}-6), 2.82\left(\mathrm{dd},{ }^{3} J=5.3 \mathrm{~Hz},{ }^{3} J=4.0 \mathrm{~Hz}, 1 \mathrm{H}, \mathrm{H}-5\right), 1.28\left(\mathrm{t},{ }^{3} J=7.1 \mathrm{~Hz}, 3 \mathrm{H}\right.$, $\left.\mathrm{CH}_{2} \mathrm{CH}_{3}\right) .{ }^{13} \mathrm{C}-\mathrm{NMR}\left(75 \mathrm{MHz}, \mathrm{CDCl}_{3}\right): \delta(\mathrm{ppm})=168.58\left(\mathrm{C}_{\mathrm{q}}, \mathrm{CO}_{2} \mathrm{Et}\right), 163.82\left(\mathrm{C}_{\mathrm{q}}, \mathrm{CO}_{2} \mathrm{Me}\right)$, $95.98\left(\mathrm{C}_{\mathrm{q}}, \mathrm{C}-3\right), 68.03(+, \mathrm{C}-1), 61.51\left(-, \mathrm{CH}_{2}\right), 54.31(+, \mathrm{C}-4), 53.52\left(+, \mathrm{OCH}_{3}\right), 31.00(+, \mathrm{C}-$ 5), $24.94(+, \mathrm{C}-6), 14.14\left(+, \mathrm{CH}_{2} \mathrm{CH}_{3}\right) . \mathbf{I R}(\mathrm{KBr}): v\left(\mathrm{~cm}^{-1}\right)=3089,3039,2990,2976,2958$, 2908, 1769, 1753, 1709, 1474, 1440, 1409, 1388, 1315, 1294, 1184, 1128, 1115, 1089, 1066, $1039,1022,1002,979,947,885,840,803,785,748,731,715,690,610 .[\alpha]_{D}{ }^{20}\left(\mathrm{CH}_{2} \mathrm{Cl}_{2}, \mathrm{c}=\right.$ 1.4) $=-298 . \quad$ MS (CI-MS): $m / z(\%)=392.1$ (5) $\left[\mathrm{M}\left({ }^{81} \mathrm{Br}\right)_{2}+\mathrm{NH}_{4}{ }^{+}\right], 390.1$ (10) $\left[\mathrm{M}\left({ }^{81} \mathrm{Br}^{79} \mathrm{Br}\right)+\mathrm{NH}_{4}{ }^{+}\right], 388.1(5)\left[\mathrm{M}\left({ }^{79} \mathrm{Br}\right)_{2}+\mathrm{NH}_{4}{ }^{+}\right], 310.1$ (7) $\left[\mathrm{M}\left({ }^{81} \mathrm{Br}\right)+\mathrm{NH}_{4}{ }^{+} \Delta \mathrm{Br}\right], 308.1$ (7) $\left[\mathrm{M}\left({ }^{79} \mathrm{Br}\right)+\mathrm{NH}_{4}{ }^{+} \Delta \mathrm{Br}\right], 230.1$ (100) $\left[\mathrm{M}+\mathrm{NH}_{4}{ }^{+} \Delta 2 \mathrm{Br}\right], 213.2$ (14) $\left[\mathrm{MH}^{+} \Delta 2 \mathrm{Br}\right] . \mathbf{C}_{\mathbf{1 0}} \mathbf{H}_{12} \mathbf{B r}_{2} \mathbf{O}_{5}$ : calcd. C 32.29, H 3.25, found C 32.54, H 3.26.

$(1 R, 5 S, 6 S)$-6-ethyl 3-methyl 4-bromo-2-oxabicyclo[3.1.0]hex-3-ene-3,6-dicarboxylate (15)

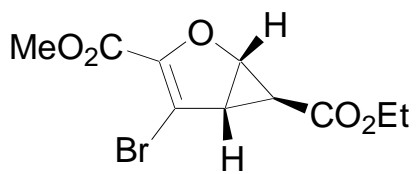

Fp: $39^{\circ} \mathrm{C}$.

${ }^{1}$ H-NMR $\left(300 \mathrm{MHz}, \mathrm{CDCl}_{3}\right): \delta(\mathrm{ppm})=4.88\left(\mathrm{dd},{ }^{3} J=5.6 \mathrm{~Hz},{ }^{3} J=1.2 \mathrm{~Hz}, 1 \mathrm{H}, \mathrm{H}-1\right), 4.15(\mathrm{q}$, $\left.{ }^{3} J=7.1 \mathrm{~Hz}, 2 \mathrm{H}, \mathrm{CH}_{2}\right), 3.82\left(\mathrm{~s}, 3 \mathrm{H}, \mathrm{OCH}_{3}\right), 3.03\left(\mathrm{dd},{ }^{3} J=5.6 \mathrm{~Hz},{ }^{3} J=2.6 \mathrm{~Hz}, 1 \mathrm{H}, \mathrm{H}-5\right)$, $1.36\left(\mathrm{dd},{ }^{3} \mathrm{~J}=2.6 \mathrm{~Hz},{ }^{3} \mathrm{~J}=1.2 \mathrm{~Hz}, 1 \mathrm{H}, \mathrm{H}-6\right), 1.26\left(\mathrm{t},{ }^{3} \mathrm{~J}=7.1 \mathrm{~Hz}, 3 \mathrm{H}, \mathrm{CH}_{2} \mathrm{CH}_{3}\right) .{ }^{13} \mathbf{C}-\mathbf{N M R}$ $\left(75 \mathrm{MHz}, \mathrm{CDCl}_{3}\right): \delta(\mathrm{ppm})=170.83\left(\mathrm{C}_{\mathrm{q}}, \underline{\mathrm{CO}_{2} \mathrm{Et}}\right), 158.82\left(\mathrm{C}_{\mathrm{q}}, \underline{\mathrm{CO}_{2} \mathrm{Me}}\right), 143.51\left(\mathrm{C}_{\mathrm{q}}, \mathrm{C}-3\right)$, $108.14\left(\mathrm{C}_{\mathrm{q}}, \mathrm{C}-4\right), 66.16(+, \mathrm{C}-1), 61.42\left(-, \mathrm{CH}_{2}\right), 52.40\left(+, \mathrm{OCH}_{3}\right), 37.54$ (+, C-5), 21.34 (+, C-6), $14.17\left(+, \mathrm{CH}_{2} \mathrm{CH}_{3}\right) . \mathbf{I R}(\mathrm{KBr}): v\left(\mathrm{~cm}^{-1}\right)=3097,3063,3036,2999,2985,2913,2870$, 2845, 1728, 1606,1483, 1437, 1403, 1378, 1354, 1326, 1298, 1271, 1180, 1171, 1139, 1103, $1075,1029,991,910,895,870,829,801,772,729,581,523,454 .[\alpha]_{\mathbf{D}}{ }^{20}\left(\mathrm{CH}_{2} \mathrm{Cl}_{2}, \mathrm{c}=1.6\right)=$ 
-154. MS (EI-MS): $m / z(\%)=292.0(15)\left[\mathrm{M}\left({ }^{81} \mathrm{Br}\right)^{+}\right], 290.0(15)\left[\mathrm{M}\left({ }^{79} \mathrm{Br}\right)^{+}\right], 247.0$ (4) $\left[\mathrm{M}\left({ }^{81} \mathrm{Br}\right)^{+} \Delta \mathrm{OEt}\right], 245.0$ (4) $\left[\mathrm{M}\left({ }^{79} \mathrm{Br}\right)^{+} \Delta \mathrm{OEt}\right], 218.9$ (99) $\left[\mathrm{M}\left({ }^{81} \mathrm{Br}\right)^{+} \Delta \mathrm{CO}_{2} \mathrm{Et}\right], 216.9$ (100) $\left[\mathrm{M}\left({ }^{79} \mathrm{Br}\right)^{+} \Delta \mathrm{CO}_{2} \mathrm{Et}\right], 211.0$ (17) $\left[\mathrm{M}^{+} \Delta \mathrm{Br}\right] . \mathbf{C}_{\mathbf{1 0}} \mathbf{H}_{11} \mathbf{B r O}_{5}$ : calcd. C 41.26, H 3.81, Br 27.45, found $\mathrm{C}: 41.42, \mathrm{H} 3.78, \mathrm{Br} 27.09$.

(1S,5R,6S)-6-ethyl 3-methyl 4-phenyl-2-oxabicyclo[3.1.0]hex-3-ene-3,6-dicarboxylate (16)

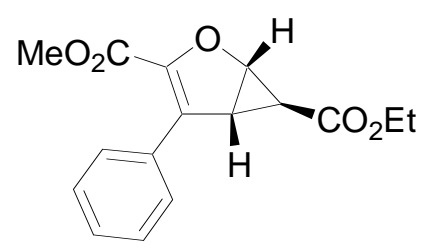

Fp: $68^{\circ} \mathrm{C}$.

${ }^{1} \mathbf{H}-\mathbf{N M R}\left(600 \mathrm{MHz}, \mathrm{CDCl}_{3}\right): \delta(\mathrm{ppm})=7.61\left(\mathrm{~m}, 2 \mathrm{H}, \mathrm{H}_{\text {arom }}\right), 7.37\left(\mathrm{~m}, 3 \mathrm{H}, \mathrm{H}_{\text {arom }}\right), 4.94$ (dd, $\left.{ }^{3} J=5.4 \mathrm{~Hz},{ }^{3} J=1.1 \mathrm{~Hz}, 1 \mathrm{H}, \mathrm{H}-1\right), 4.21\left(\mathrm{dq},{ }^{2} J=10.8 \mathrm{~Hz},{ }^{3} J=7.2 \mathrm{~Hz}, 1 \mathrm{H}, \mathrm{C} \underline{\mathrm{H}}-\mathrm{CH}_{3}\right), 4.17$ $\left(\mathrm{dq},{ }^{2} J=10.8 \mathrm{~Hz},{ }^{3} J=7.1 \mathrm{~Hz}, 1 \mathrm{H}, \mathrm{CH} \underline{\mathrm{H}}-\mathrm{CH}_{3}\right), 3.78\left(\mathrm{~s}, 3 \mathrm{H}, \mathrm{OCH}_{3}\right), 3.11\left(\mathrm{dd},{ }^{3} J=5.4 \mathrm{~Hz},{ }^{3} J\right.$ $=2.7 \mathrm{~Hz}, 1 \mathrm{H}, \mathrm{H}-5), 1.41\left(\mathrm{dd},{ }^{3} J=2.7 \mathrm{~Hz},{ }^{3} J=1.1 \mathrm{~Hz}, 1 \mathrm{H}, \mathrm{H}-6\right), 1.29\left(\mathrm{dd},{ }^{3} J=7.2 \mathrm{~Hz},{ }^{3} J=7.1\right.$ $\left.\mathrm{Hz}, 3 \mathrm{H}, \mathrm{CH}_{2} \mathrm{CH}_{3}\right) .{ }^{13} \mathrm{C}-\mathrm{NMR}\left(151 \mathrm{MHz}, \mathrm{CDCl}_{3}\right): \delta(\mathrm{ppm})=171.75\left(\mathrm{C}_{\mathrm{q}}, \underline{\mathrm{CO}}_{2} \mathrm{Et}\right), 160.11\left(\mathrm{C}_{\mathrm{q}}\right.$, $\left.\underline{\mathrm{CO}}_{2} \mathrm{Me}\right), 140.28\left(\mathrm{C}_{\mathrm{q}}, \mathrm{C}-3\right), 131.23\left(\mathrm{C}_{\mathrm{q}}, \mathrm{C}-4\right), 131.19\left(\mathrm{C}_{\mathrm{q}}, \mathrm{C}_{\text {arom }}\right), 129.04\left(+, 2 \mathrm{C}, \mathrm{C}_{\mathrm{arom}}\right)$, $128.87\left(+, \mathrm{C}_{\text {arom }}\right), 128.06\left(+, 2 \mathrm{C}, \mathrm{C}_{\text {arom }}\right), 64.73(+, \mathrm{C}-1), 61.20\left(-, \mathrm{CH}_{2}\right), 52.10\left(+, \mathrm{OCH}_{3}\right)$, 36.37 (+, C-5), $21.68(+, \mathrm{C}-6), 14.24\left(+, \mathrm{CH}_{2} \mathrm{CH}_{3}\right) . \mathbf{I R}(\mathrm{KBr}): v\left(\mathrm{~cm}^{-1}\right)=3064,2984,2956$, 2906, 1712, 1616, 1495, 1437, 1400, 1381, 1354, 1304, 1263, 1173, 1138, 1096, 1082, 1041, 998, 986, 936, 878, 843, 807, 763, 743, 691, 667, 642, 613. $[\alpha]_{\mathbf{D}}{ }^{20}\left(\mathrm{CH}_{2} \mathrm{Cl}_{2}, \mathrm{c}=1.3\right)=-103$. MS (EI-MS): $m / z(\%)=288.0(1)\left[\mathrm{M}^{+}\right], 243.0$ (5) $\left[\mathrm{M}^{+} \Delta \mathrm{OEt}\right], 215.0$ (63) $\left[\mathrm{M}^{+} \Delta \mathrm{CO}_{2} \mathrm{Et}\right], 201.0$ (100) $\left[\mathrm{M}^{+} \Delta \mathrm{CH}_{2} \mathrm{CO}_{2} \mathrm{Et}\right], 173.0$ (52) $\left[\mathrm{M}^{+} \Delta \mathrm{CH}_{2} \mathrm{CO}_{2} \mathrm{Et} \Delta \mathrm{CO}\right], 155.0$ (15), 128.0 (19), 127.0 (19), 115.0 (10), 102.0 (11), $77.0(6)\left[\mathrm{Ph}^{+}\right] . \mathbf{C}_{\mathbf{1 6}} \mathbf{H}_{16} \mathbf{O}_{5}$ : calcd. C 66.66, H 5.59, found C 66.53, H 5.41 . 
$(1 S, 5 R, 6 S)$-6-ethyl

dicarboxylate (17)

Fp: $115^{\circ} \mathrm{C}$.

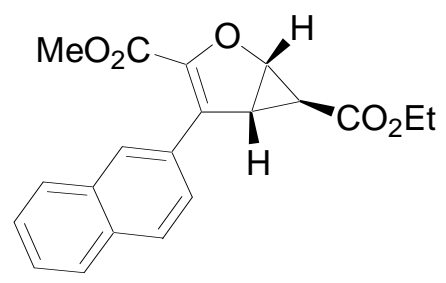

${ }^{1}$ H-NMR $\left(300 \mathrm{MHz}, \mathrm{CDCl}_{3}\right): \delta(\mathrm{ppm})=8.08-8.06\left(\mathrm{~m}, 1 \mathrm{H}, \mathrm{H}_{\text {arom }}\right), 7.90-7.79(\mathrm{~m}, 3 \mathrm{H}$, $\left.\mathrm{H}_{\text {arom }}\right), 7.71\left(\mathrm{dd},{ }^{3} J=8.6 \mathrm{~Hz},{ }^{4} J=1.8 \mathrm{~Hz}, 1 \mathrm{H}, \mathrm{H}_{\text {arom }}\right), 7.53-7.46\left(\mathrm{~m}, 2 \mathrm{H}, \mathrm{H}_{\text {arom }}\right), 4.98\left(\mathrm{dd},{ }^{3} J\right.$ $\left.=5.4 \mathrm{~Hz},{ }^{3} J=1.1 \mathrm{~Hz}, 1 \mathrm{H}, \mathrm{H}-1\right), 4.24\left(\mathrm{dq},{ }^{2} J=10.8 \mathrm{~Hz},{ }^{3} J=7.1 \mathrm{~Hz}, 1 \mathrm{H}, \mathrm{CHHCH}_{3}\right), 4.20$ $\left(\mathrm{dq},{ }^{2} J=10.8 \mathrm{~Hz},{ }^{3} J=7.1 \mathrm{~Hz}, 1 \mathrm{H}, \mathrm{CHHCH}_{3}\right), 3.79\left(\mathrm{~s}, 3 \mathrm{H}, \mathrm{CO}_{2} \mathrm{CH}_{3}\right), 3.21\left(\mathrm{dd},{ }^{3} J=5.4 \mathrm{~Hz}\right.$, $\left.{ }^{3} J=2.7 \mathrm{~Hz}, 1 \mathrm{H}, \mathrm{H}-5\right), 1.49\left(\mathrm{dd},{ }^{3} J=2.7 \mathrm{~Hz},{ }^{3} J=1.1 \mathrm{~Hz}, 1 \mathrm{H}, \mathrm{H}-6\right), 1.31\left(\mathrm{dd},{ }^{3} J=7.1 \mathrm{~Hz},{ }^{3} J\right.$ $\left.=7.1 \mathrm{~Hz}, 3 \mathrm{H}, \mathrm{CH}_{2} \underline{\mathrm{CH}}_{3}\right) \cdot{ }^{13} \mathrm{C}-\mathrm{NMR}\left(75 \mathrm{MHz}, \mathrm{CDCl}_{3}\right): \delta(\mathrm{ppm})=171.86\left(\mathrm{C}_{\mathrm{q}}, \underline{\mathrm{CO}}_{2} \mathrm{Et}\right), 160.19$ $\left(\mathrm{C}_{\mathrm{q}}, \underline{\mathrm{CO}}_{2} \mathrm{Me}\right), 140.48\left(\mathrm{C}_{\mathrm{q}}, \mathrm{C}-3\right), 133.30\left(\mathrm{C}_{\mathrm{q}}\right), 132.89\left(\mathrm{C}_{\mathrm{q}}\right), 131.30\left(\mathrm{C}_{\mathrm{q}}\right), 128.69\left(\mathrm{C}_{\mathrm{q}}\right), 128.64(+$, $\left.\mathrm{C}_{\text {arom }}\right), 128.35\left(+, \mathrm{C}_{\text {arom }}\right), 127.68\left(+, \mathrm{C}_{\text {arom }}\right), 127.51\left(+, \mathrm{C}_{\text {arom }}\right), 126.82\left(+, \mathrm{C}_{\text {arom }}\right), 126.63(+$,

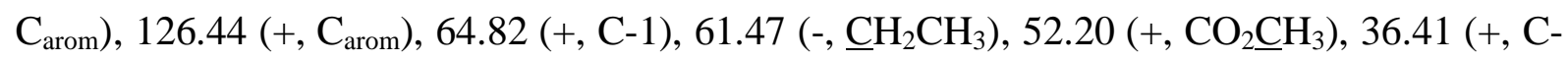
5), $21.83(+, \mathrm{C}-6), 14.29\left(+, \mathrm{CH}_{2} \mathrm{CH}_{3}\right) . \mathbf{I R}(\mathrm{KBr}): v\left(\mathrm{~cm}^{-1}\right)=3067,2988,2950,2905,1713$, 1611, 1437, 1399, 1377, 1343, 1302, 1269, 1171, 1137, 1094, 1077, 1040, 995, 935, 857, 840, 816, 746. $[\alpha]_{\mathbf{D}}{ }^{20}\left(\mathrm{CH}_{2} \mathrm{Cl}_{2}, \mathrm{c}=0.3\right)=-24$. MS (EI-MS): $m / z(\%): 338.2(9)\left[\mathrm{M}^{+}\right], 265.2(73)$ $\left[\mathrm{M}^{+} \Delta \mathrm{CO}_{2} \mathrm{Et}\right], 251.2(100)\left[\mathrm{M}^{+} \Delta \mathrm{CH}_{2} \mathrm{CO}_{2} \mathrm{Et}\right], 223.2$ (60) $\left[\mathrm{M}^{+} \Delta \mathrm{CH}_{2} \mathrm{CO}_{2} \mathrm{Et} \Delta \mathrm{CO}\right], 205.2$ (38), 178.2 (22), 155.1 (27), 152.1 (21), $127.1(5)\left[\mathrm{C}_{10} \mathrm{H}_{7}{ }^{+}\right], 95.1$ (8). $\mathbf{C}_{20} \mathbf{H}_{18} \mathbf{O}_{5}$ : calcd. C 70.99, H 5.36, found C 70.66, H 5.33 .

$(1 S, 5 R, 6 S)$-6-ethyl 3-methyl 4-(2-methoxyphenyl)-2-oxabicyclo[3.1.0]hex-3-ene-3,6dicarboxylate (18)

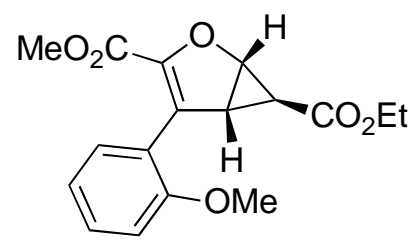

Fp: $76^{\circ} \mathrm{C}$.

${ }^{1}$ H-NMR $\left(300 \mathrm{MHz}\right.$, Aceton- $\left.\mathrm{d}_{6}\right): \delta(\mathrm{ppm})=7.38-7.31\left(\mathrm{~m}, 2 \mathrm{H}, \mathrm{H}_{\text {arom }}\right), 7.06-6.93(\mathrm{~m}, 2 \mathrm{H}$, $\mathrm{H}_{\text {arom }}$ ), $4.97\left(\mathrm{dd},{ }^{3} J=5.3 \mathrm{~Hz},{ }^{3} J=1.1 \mathrm{~Hz}, 1 \mathrm{H}, \mathrm{H}-1\right), 4.16\left(\mathrm{dq},{ }^{2} J=10.9 \mathrm{~Hz},{ }^{3} J=7.1 \mathrm{~Hz}, 1 \mathrm{H}\right.$, $\left.\mathrm{CHHCH}_{3}\right), 4.12\left(\mathrm{dq},{ }^{2} J=10.9 \mathrm{~Hz},{ }^{3} J=7.1 \mathrm{~Hz}, 1 \mathrm{H}, \mathrm{CH}^{\mathrm{H} C H}{ }_{3}\right), 3.84\left(\mathrm{~s}, 3 \mathrm{H}, \mathrm{CO}_{2} \mathrm{CH}_{3}\right), 3.63$ (s, $\left.3 \mathrm{H}, \mathrm{Ar}-\mathrm{OCH}_{3}\right), 3.04\left(\mathrm{dd},{ }^{3} J=5.3 \mathrm{~Hz},{ }^{3} J=2.6 \mathrm{~Hz}, 1 \mathrm{H}, \mathrm{H}-5\right), 1.35\left(\mathrm{dd},{ }^{3} \mathrm{~J}=2.6 \mathrm{~Hz},{ }^{3} J=\right.$ 
$1.1 \mathrm{~Hz}, 1 \mathrm{H}, \mathrm{H}-6), 1.24\left(\mathrm{dd},{ }^{3} J=7.1 \mathrm{~Hz},{ }^{3} J=7.1 \mathrm{~Hz}, 3 \mathrm{H}, \mathrm{CH}_{2} \mathrm{C}_{3}\right) .{ }^{13} \mathbf{C}-\mathrm{NMR}(75 \mathrm{MHz}$, Aceton- $\left.\mathrm{d}_{6}\right): \delta(\mathrm{ppm})=172.19\left(\mathrm{C}_{\mathrm{q}}, \underline{\mathrm{CO}} \mathrm{Et}\right), 160.15\left(\mathrm{C}_{\mathrm{q}}, \underline{\mathrm{CO}_{2} \mathrm{Me}}\right), 157.14\left(\mathrm{C}_{\mathrm{q}}, \mathrm{C}_{\text {arom }} \mathrm{OMe}\right)$, $142.02\left(\mathrm{C}_{\mathrm{q}}, \mathrm{C}-3\right), 130.95$ (+, $\left.\mathrm{CH}_{\text {arom }}\right), 130.07$ (+, $\left.\mathrm{CH}_{\text {arom }}\right), 128.37\left(\mathrm{C}_{\mathrm{q}}, \mathrm{C}-4\right), 120.81\left(\mathrm{C}_{\mathrm{q}}, \underline{\mathrm{C}}_{\text {arom }}{ }^{-}\right.$ C-4), 120.11 (+, $\left.\mathrm{CH}_{\text {arom }}\right), 110.68$ (+, $\left.\mathrm{CH}_{\text {arom }}\right), 66.01(+, \mathrm{C}-1), 60.96\left(-, \mathrm{CH}_{2}\right), 55.43(+, \mathrm{Ar}-$ $\left.\mathrm{O}^{\mathrm{C}} \mathrm{H}_{3}\right), 51.95\left(+, \mathrm{CO}_{2} \underline{\mathrm{CH}}_{3}\right), 36.92(+, \mathrm{C}-5), 22.06(+, \mathrm{C}-6), 14.30\left(+, \mathrm{CH}_{2} \underline{\mathrm{CH}}_{3}\right) . \mathrm{IR}(\mathrm{KBr}): v$ $\left(\mathrm{cm}^{-1}\right)=3067,3008,2985,2964,2946,2877,1719,1707,1617,1598,1493,1462,1444$, 1402, 1381, 1356, 1305, 1258, 1200, 1179, 1138, 1098, 1078, 1034, 993, 940, 908, 876, 840, $811,792,781,753,650 .[\alpha]_{\mathbf{D}}{ }^{20}\left(\mathrm{CH}_{2} \mathrm{Cl}_{2}, \mathrm{c}=0.5\right)=-102$. MS $(\mathrm{EI}-\mathrm{MS}): \mathrm{m} / z(\%)=318.1(1)$ [M $\left.\mathrm{M}^{+}\right], 287.1$ (2) $\left[\mathrm{M}^{+} \Delta \mathrm{OMe}\right], 245.1$ (51) $\left[\mathrm{M}^{+} \Delta \mathrm{CO}_{2} \mathrm{Et}\right], 231.1$ (100) $\left[\mathrm{M}^{+} \Delta \mathrm{CH}_{2} \mathrm{CO}_{2} \mathrm{Et}\right], 203.1$ (35) $\left[\mathrm{M}^{+} \Delta \mathrm{CH}_{2} \mathrm{CO}_{2} \mathrm{Et} \Delta \mathrm{CO}\right], 135.0$ (87), 114.9 (22). HRMS (EI-MS): für $\mathrm{C}_{17} \mathrm{H}_{18} \mathrm{O}_{6}\left(\mathrm{M}^{+}\right)$calcd. 318.1103, found 318.1100. $\mathbf{C}_{17} \mathbf{H}_{18} \mathbf{O}_{6}$ : calcd. C 64.14, H 5.70, found C 64.15, H 5.73.

\section{$(1 S, 5 R, 6 S)$-6-ethyl 3-methyl 4 -(E)-styryl-2-oxabicyclo[3.1.0]hex-3-ene-3,6-dicarboxylate} (19)

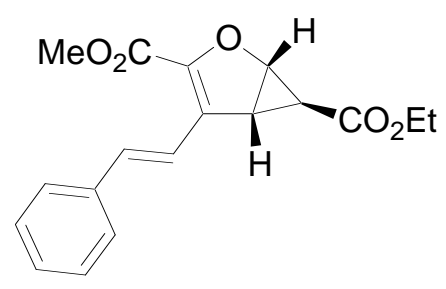

Fp: $85-88^{\circ} \mathrm{C}$.

${ }^{1}$ H-NMR $\left(300 \mathrm{MHz}, \mathrm{CDCl}_{3}\right): \delta(\mathrm{ppm})=7.72\left(\mathrm{~d},{ }^{3} J=16.2 \mathrm{~Hz}, 1 \mathrm{H}, \mathrm{CHPh}\right), 7.54-7.49$ (m, 2 $\left.\mathrm{H}, \mathrm{H}_{\text {arom }}\right), 7.39-7.25$ (m, $\left.3 \mathrm{H}, \mathrm{H}_{\text {arom }}\right), 6.91\left(\mathrm{~d},{ }^{3} J=16.2 \mathrm{~Hz}, 1 \mathrm{H}, \mathrm{C} \underline{\mathrm{H}}=\mathrm{CHPh}\right), 4.92$ (dd, ${ }^{3} J=$ $\left.5.5 \mathrm{~Hz},{ }^{3} J=1.0 \mathrm{~Hz}, 1 \mathrm{H}, \mathrm{H}-1\right), 4.23\left(\mathrm{dq},{ }^{2} J=10.8 \mathrm{~Hz},{ }^{3} J=7.1 \mathrm{~Hz}, 1 \mathrm{H}, \mathrm{CHHCH}_{3}\right), 4.19$ (dq, $\left.{ }^{2} J=10.8 \mathrm{~Hz},{ }^{3} J=7.2 \mathrm{~Hz}, 1 \mathrm{H}, \mathrm{CH}{ }^{2} \mathrm{CH}_{3}\right), 3.88\left(\mathrm{~s}, 3 \mathrm{H}, \mathrm{CO}_{2} \mathrm{CH}_{3}\right), 3.19\left(\mathrm{dd},{ }^{3} J=5.5 \mathrm{~Hz},{ }^{3} J=\right.$ $2.9 \mathrm{~Hz}, 1 \mathrm{H}, \mathrm{H}-5), 1.34\left(\mathrm{dd},{ }^{3} J=2.9 \mathrm{~Hz},{ }^{3} J=1.0 \mathrm{~Hz}, 1 \mathrm{H}, \mathrm{H}-6\right), 1.31\left(\mathrm{dd},{ }^{3} J=7.2 \mathrm{~Hz},{ }^{3} J=7.1\right.$ $\left.\mathrm{Hz}, 3 \mathrm{H}, \mathrm{CH}_{2} \underline{\mathrm{C}}_{3}\right) .{ }^{13} \mathrm{C}-\mathrm{NMR}\left(75 \mathrm{MHz}, \mathrm{CDCl}_{3}\right): \delta(\mathrm{ppm})=171.88\left(\mathrm{C}_{\mathrm{q}}, \underline{\mathrm{CO}}_{2} \mathrm{Et}\right), 160.36\left(\mathrm{C}_{\mathrm{q}}\right.$, $\left.\mathrm{CO}_{2} \mathrm{Me}\right), 141.84\left(\mathrm{C}_{\mathrm{q}}, \mathrm{C}-3\right), 136.61\left(\mathrm{C}_{\mathrm{q}}, \mathrm{C}_{\text {arom }}\right), 134.44(+, \underline{\mathrm{H} P h}), 131.32\left(\mathrm{C}_{\mathrm{q}}, \mathrm{C}-4\right), 128.80$ (+, $\left.2 \mathrm{C}, \mathrm{C}_{\text {arom}}\right), 128.53$ (+, $\left.\mathrm{C}_{\text {arom }}\right), 127.03$ (+, $\left.2 \mathrm{C}, \mathrm{C}_{\text {arom}}\right), 119.59$ (+, $\left.\underline{\mathrm{C}} \mathrm{H}=\mathrm{CHPh}\right), 65.66$ (+, C-1), 61.29 (-, $\left.\mathrm{CH}_{2} \mathrm{CH}_{3}\right), 52.16$ (+, $\left.\mathrm{CO}_{2} \mathrm{CH}_{3}\right), 31.31$ (+, C-5), 22.18 (+, C-6), $14.24\left(+, \mathrm{CH}_{2} \mathrm{CH}_{3}\right)$. IR $(\mathrm{KBr}): v\left(\mathrm{~cm}^{-1}\right)=3062,3026,2986,2945,2904,2871,2846,1713,1618,1582,1496$, 1476, 1448, 1436, 1402, 1377, 1308, 1290, 1259, 1230, 1184, 1165, 1131, 1089, 1067, 1039, $996,987,944,926,895,879,839,814,778,763,751,690,622 .[\alpha]_{D}{ }^{20}\left(\mathrm{CH}_{2} \mathrm{Cl}_{2}, \mathrm{c}=1.0\right)=-$ 30. MS (EI-MS): $m / z(\%)=314.1\left[\mathrm{M}^{+}\right]$(23), $241.1\left[\mathrm{M}^{+} \Delta \mathrm{CO}_{2} \mathrm{Et}\right]$ (78), 227.1 (67) 
$\left[\mathrm{M}^{+} \Delta \mathrm{CH}_{2} \mathrm{CO}_{2} \mathrm{Et}\right], 181.1$ (100), 153.1 (64). $\mathbf{C}_{\mathbf{1 8}} \mathbf{H}_{18} \mathbf{O}_{5}$ : calcd. C 68.78, H 5.77, found $\mathrm{C}$ 68.85, H 5.52 .

$(1 S, 3 R, 4 R, 5 S, 6 S)$-6-ethyl 3-methyl 4-phenyl-2-oxabicyclo[3.1.0]hexane-3,6-dicarboxylate (20)

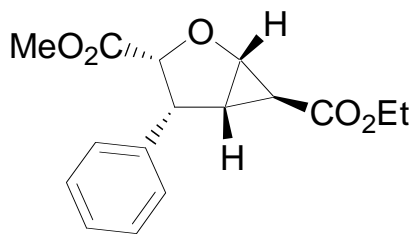

Fp: $67-69^{\circ} \mathrm{C}$

${ }^{1} \mathbf{H}-\mathbf{N M R}\left(300 \mathrm{MHz}, \mathrm{CDCl}_{3}\right): \delta(\mathrm{ppm})=7.39-7.19\left(\mathrm{~m}, 5 \mathrm{H}, \mathrm{H}_{\text {arom }}\right), 5.01\left(\mathrm{dd},{ }^{3} J=11.0 \mathrm{~Hz},{ }^{4} J\right.$ $=0.4 \mathrm{~Hz}, 1 \mathrm{H}, \mathrm{H}-3), 4.40\left(\mathrm{ddd},{ }^{3} J=5.6 \mathrm{~Hz},{ }^{3} J=1.0 \mathrm{~Hz},{ }^{4} J=0.4 \mathrm{~Hz}, 1 \mathrm{H}, \mathrm{H}-1\right), 4.35\left(\mathrm{dd},{ }^{3} J=\right.$ $\left.11.0 \mathrm{~Hz},{ }^{3} J=5.2 \mathrm{~Hz}, 1 \mathrm{H}, \mathrm{H}-4\right), 4.17\left(\mathrm{dd},{ }^{2} J=10.8 \mathrm{~Hz},{ }^{3} J=7.1 \mathrm{~Hz}, 1 \mathrm{H}, \mathrm{CHHCH}_{3}\right), 4.11$ (dd, $\left.{ }^{2} J=10.8 \mathrm{~Hz},{ }^{3} J=7.1 \mathrm{~Hz}, 1 \mathrm{H}, \mathrm{CHHCH}_{3}\right), 3.13\left(\mathrm{~s}, 3 \mathrm{H}, \mathrm{OCH}_{3}\right), 3.13\left(\mathrm{dd},{ }^{3} J=4.5 \mathrm{~Hz},{ }^{3} J=1.0\right.$ $\mathrm{Hz}, 1 \mathrm{H}, \mathrm{H}-6), 2.42\left(\mathrm{ddd},{ }^{3} J=5.6 \mathrm{~Hz},{ }^{3} J=5.2 \mathrm{~Hz},{ }^{3} J=4.5 \mathrm{~Hz}, 1 \mathrm{H}, \mathrm{H}-5\right), 1.28\left(\mathrm{dd},{ }^{3} J=7.1\right.$ $\left.\mathrm{Hz},{ }^{3} \mathrm{~J}=7.1 \mathrm{~Hz}, 3 \mathrm{H}, \mathrm{CH}_{2} \underline{\mathrm{C}}_{3}\right) .{ }^{13} \mathbf{C}-\mathbf{N M R}\left(75 \mathrm{MHz}, \mathrm{CDCl}_{3}\right): \delta(\mathrm{ppm})=170.84\left(\mathrm{C}_{\mathrm{q}}, \underline{\mathrm{CO}}_{2} \mathrm{Me}\right)$,

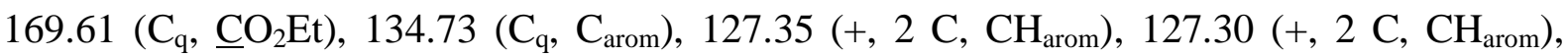
126.60 (+, $\left.\mathrm{CH}_{\text {arom }}\right), 81.36$ (+, C-3), 64.71 (+, C-1), 59.68 (-, $\left.\mathrm{CH}_{2} \mathrm{CH}_{3}\right), 50.39$ (+, $\left.\mathrm{OCH}_{3}\right)$, 47.33 (+, C-4), 28.47 (+, C-5), $25.12(+, \mathrm{C}-6), 13.24\left(+, \mathrm{CH}_{2} \underline{\mathrm{CH}}_{3}\right) . \mathbf{I R}(\mathrm{KBr}): v\left(\mathrm{~cm}^{-1}\right)=3083$, 3066, 3035, 3003, 2985, 2975, 2948, 2932, 2911, 1749, 1713, 1497, 1476, 1456, 1434, 1410, 1376, 1351, 1321, 1293, 1281, 1215, 1197, 1173, 1121, 1091, 1076, 1065, 1046, 1030, 999, 967, 937, 904, 888, 875, 848, 812, 759, 704. $[\alpha]_{\mathbf{D}}{ }^{20}\left(\mathrm{CH}_{2} \mathrm{Cl}_{2}, \mathrm{c}=1.0\right)=-54$. MS (EI-MS): $m / z(\%)=290.0(1)\left[\mathrm{M}^{+}\right], 162.0$ (100), 131.9 (45), 129.0 (26). $\mathbf{C}_{16} \mathbf{H}_{18} \mathbf{O}_{5}$ : calcd. C 66.19, H 6.25 , found C 66.08, H 6.17.

$(1 S, 3 R, 4 R, 5 S, 6 S)$-6-ethyl 3-methyl 4-(naphthalen-2-yl)-2-oxabicyclo[3.1.0]hexane-3,6dicarboxylate (21)

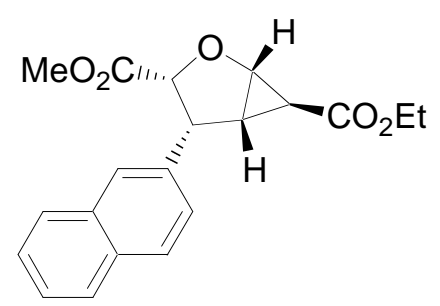

Fp: $69-70^{\circ} \mathrm{C}$.

${ }^{1} \mathbf{H}-\mathbf{N M R}\left(600 \mathrm{MHz}, \mathrm{CDCl}_{3}\right): \delta(\mathrm{ppm})=7.84\left(\mathrm{~d},{ }^{4} \mathrm{~J}=1.7 \mathrm{~Hz}, 1 \mathrm{H}, \mathrm{H}_{\text {arom }}\right), 7.82-7.79(\mathrm{~m}, 2 \mathrm{H}$, $\left.\mathrm{H}_{\text {arom }}\right), 7.78\left(\mathrm{~d},{ }^{3} \mathrm{~J}=8.5 \mathrm{~Hz}, 1 \mathrm{H}, \mathrm{H}_{\text {arom }}\right), 7.50-7.46$ (m, $\left.2 \mathrm{H}, \mathrm{H}_{\text {arom }}\right), 7.40\left(\mathrm{dd},{ }^{3} J=8.5 \mathrm{~Hz},{ }^{4} \mathrm{~J}=\right.$ 
$\left.1.7 \mathrm{~Hz}, 1 \mathrm{H}, \mathrm{H}_{\text {arom }}\right), 5.12\left(\mathrm{~d},{ }^{3} \mathrm{~J}=11.0 \mathrm{~Hz}, 1 \mathrm{H}, \mathrm{H}-3\right), 4.53\left(\mathrm{dd},{ }^{3} \mathrm{~J}=11.0 \mathrm{~Hz},{ }^{3} \mathrm{~J}=5.2 \mathrm{~Hz}, 1 \mathrm{H}\right.$, H-4), 4.47 (dd, $\left.{ }^{3} J=5.6 \mathrm{~Hz},{ }^{3} J=1.0 \mathrm{~Hz}, 1 \mathrm{H}, \mathrm{H}-1\right), 4.22\left(\mathrm{dq},{ }^{2} J=10.8 \mathrm{~Hz},{ }^{3} J=7.2 \mathrm{~Hz}, 1 \mathrm{H}\right.$, $\left.\mathrm{CHHCH}_{3}\right), 4.16\left(\mathrm{dq},{ }^{2} J=10.8 \mathrm{~Hz},{ }^{3} J=7.1 \mathrm{~Hz}, 1 \mathrm{H}, \mathrm{CHHCH}_{3}\right), 3.22\left(\mathrm{dd},{ }^{3} J=4.4 \mathrm{~Hz},{ }^{3} J=1.0\right.$ $\mathrm{Hz}, 1 \mathrm{H}, \mathrm{H}-6), 3.04$ (s, $3 \mathrm{H}, \mathrm{CO}_{2} \mathrm{CH}_{3}$ ), $2.52\left(\mathrm{ddd},{ }^{3} \mathrm{~J}=5.6 \mathrm{~Hz},{ }^{3} J=5.2 \mathrm{~Hz},{ }^{3} J=4.4 \mathrm{~Hz}, 1 \mathrm{H}\right.$, $\mathrm{H}-5), 1.32\left(\mathrm{dd},{ }^{3} J=7.2 \mathrm{~Hz},{ }^{3} J=7.1 \mathrm{~Hz}, 3 \mathrm{H}, \mathrm{CH}_{2} \mathrm{CH}_{3}\right) .{ }^{13} \mathbf{C}-\mathbf{N M R}\left(75 \mathrm{MHz}, \mathrm{CDCl}_{3}\right): \delta(\mathrm{ppm})$ $=171.86\left(\mathrm{C}_{\mathrm{q}}, \underline{\mathrm{CO}_{2} \mathrm{Me}}\right), 170.69\left(\mathrm{C}_{\mathrm{q}}, \underline{\mathrm{CO}}_{2} \mathrm{Et}\right), 133.29\left(\mathrm{C}_{\mathrm{q}}, \mathrm{C}_{\mathrm{arom}}\right), 133.14\left(\mathrm{C}_{\mathrm{q}}, \mathrm{C}_{\mathrm{arom}}\right), 132.69$ $\left(\mathrm{C}_{\mathrm{q}}, \mathrm{C}_{\text {arom }}\right), 127.96\left(+, \mathrm{C}_{\text {arom }}\right), 127.78\left(+, \mathrm{C}_{\text {arom }}\right), 127.56\left(+, \mathrm{C}_{\text {arom }}\right), 126.99\left(+, \mathrm{C}_{\text {arom }}\right), 126.71$ (+, $\left.\mathrm{C}_{\text {arom }}\right), 126.28$ (+, $\left.\mathrm{C}_{\text {arom}}\right), 126.11$ (+, $\left.\mathrm{C}_{\text {arom }}\right), 82.36$ (+, C-3), 65.83 (+, C-1), 60.78 (-, $\mathrm{CH}_{2} \mathrm{CH}_{3}$ ), 51.46 (+, $\left.\mathrm{CO}_{2} \mathrm{CH}_{3}\right), 48.59$ (+, C-4), 29.65 (+, C-5), 26.29 (+, C-6), 14.33 (+, $\left.\mathrm{CH}_{2} \mathrm{CH}_{3}\right) . \mathbf{I R}(\mathrm{KBr}): v\left(\mathrm{~cm}^{-1}\right)=3079,3051,2992,2950,1746,1717,1509,1436,1417,1366$, 1324, 1287, 1271, 1215, 1185, 1117, 1078, 1066, 1049, 1033, 1013, 973, 935, 893, 854, 827, 798, 745, 699. $[\boldsymbol{\alpha}]_{\mathbf{D}}{ }^{20}\left(\mathrm{CH}_{2} \mathrm{Cl}_{2}, \mathrm{c}=0.6\right)=-60 . \mathrm{MS}(\mathrm{EI}-\mathrm{MS}): \mathrm{m} / \mathrm{z}(\%)=340.2(9)\left[\mathrm{M}^{+}\right], 212.1$ (100) $\left[\mathrm{C}_{10} \mathrm{H}_{7}-\mathrm{CH}=\mathrm{CH}-\mathrm{CO}_{2} \mathrm{Me}^{+}\right], 181.1$ (34) $\left[\mathrm{C}_{10} \mathrm{H}_{7}-\mathrm{CH}=\mathrm{CH}-\mathrm{CO}^{+}\right], 179.1$ (38), 178.1 (30), 165.1 (18), 153.1 (15) $\left[\mathrm{C}_{10} \mathrm{H}_{7}-\mathrm{CH}=\mathrm{CH}^{+}\right], 152.1$ (21), 141.1 (13), 128.1 (7), 127.1 (5) $\left[\mathrm{C}_{10} \mathrm{H}_{7}^{+}\right]$. $\mathbf{C}_{20} \mathbf{H}_{20} \mathbf{O}_{5}$ : calcd. C 70.57, H 5.92, found C 70.58, H 5.86.

$(1 S, 3 R, 4 S, 5 S, 6 S)$-6-ethyl 3-methyl 4-phenethyl-2-oxabicyclo[3.1.0]hexane-3,6-dicarboxylate (22)

Fp: $67-71^{\circ} \mathrm{C}$.

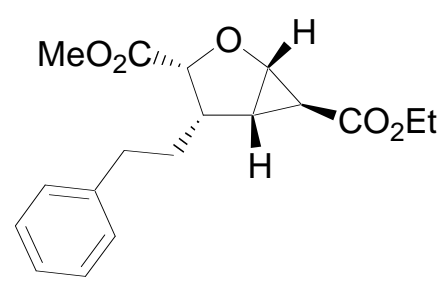

${ }^{1} \mathbf{H}-\mathbf{N M R}\left(600 \mathrm{MHz}, \mathrm{CDCl}_{3}\right): \delta(\mathrm{ppm})=7.29\left(\mathrm{dd},{ }^{3} \mathrm{~J}=7.7 \mathrm{~Hz},{ }^{3} \mathrm{~J}=7.4 \mathrm{~Hz}, 2 \mathrm{H}, \mathrm{H}_{\text {arom-meta }}\right)$, $7.20\left(\mathrm{t},{ }^{3} J=7.4 \mathrm{~Hz}, 1 \mathrm{H}, \mathrm{H}_{\text {arom-para }}\right), 7.18$ (d, $\left.{ }^{3} J=7.7 \mathrm{~Hz}, 2 \mathrm{H}, \mathrm{H}_{\text {arom-ortho }}\right), 4.78$ (d, ${ }^{3} J=10.4$ $\mathrm{Hz}, 1 \mathrm{H}, \mathrm{H}-3), 4.30\left(\mathrm{dd},{ }^{3} J=5.7 \mathrm{~Hz},{ }^{3} J=1.1 \mathrm{~Hz}, 1 \mathrm{H}, \mathrm{H}-1\right), 4.14\left(\mathrm{dq},{ }^{2} J=10.8 \mathrm{~Hz},{ }^{3} J=7.1\right.$ $\left.\mathrm{Hz}, 1 \mathrm{H}, \mathrm{C}_{\mathrm{HHCH}}\right), 4.08\left(\mathrm{dq},{ }^{2} \mathrm{~J}=10.8 \mathrm{~Hz},{ }^{3} \mathrm{~J}=7.1 \mathrm{~Hz}, 1 \mathrm{H}, \mathrm{CH} \mathrm{HCH}_{3}\right), 3.71(\mathrm{~s}, 3 \mathrm{H}$, $\mathrm{CO}_{2} \mathrm{CH}_{3}$ ), 3.01 (dddd, ${ }^{3} \mathrm{~J}=11.3 \mathrm{~Hz},{ }^{3} \mathrm{~J}=10.4 \mathrm{~Hz},{ }^{3} \mathrm{~J}=5.6 \mathrm{~Hz},{ }^{3} J=4.4 \mathrm{~Hz}, 1 \mathrm{H}, \mathrm{H}-4$ ), 2.80 $\left(\mathrm{ddd},{ }^{2} J=13.9 \mathrm{~Hz},{ }^{3} J=8.9 \mathrm{~Hz},{ }^{3} J=5.6 \mathrm{~Hz}, 1 \mathrm{H}, \mathrm{CHHPh}\right), 2.65\left(\mathrm{ddd},{ }^{2} J=13.9 \mathrm{~Hz},{ }^{3} J=8.6\right.$ $\mathrm{Hz},{ }^{3} J=7.2 \mathrm{~Hz}, 1 \mathrm{H}, \mathrm{CH} \underline{\mathrm{HPh}}$ ), $2.34\left(\mathrm{dd},{ }^{3} J=4.3 \mathrm{~Hz},{ }^{3} J=1.1 \mathrm{~Hz}, 1 \mathrm{H}, \mathrm{H}-6\right), 2.28$ (ddd, ${ }^{3} J=$ $\left.5.7 \mathrm{~Hz},{ }^{3} J=5.6 \mathrm{~Hz},{ }^{3} J=4.3 \mathrm{~Hz}, 1 \mathrm{H}, \mathrm{H}-5\right), 1.85$ (dddd, ${ }^{2} J=13.3 \mathrm{~Hz},{ }^{3} J=8.9 \mathrm{~Hz},{ }^{3} J=7.2 \mathrm{~Hz}$, $\left.{ }^{3} J=4.4 \mathrm{~Hz}, 1 \mathrm{H}, \mathrm{CHHCH}_{2} \mathrm{Ph}\right), 1.51\left(\mathrm{dddd},{ }^{2} J=13.3 \mathrm{~Hz},{ }^{3} J=11.3 \mathrm{~Hz},{ }^{3} J=8.6 \mathrm{~Hz},{ }^{3} J=5.6\right.$ $\left.\mathrm{Hz}, 1 \mathrm{H}, \mathrm{CH} \underline{\mathrm{HCH}}{ }_{2} \mathrm{Ph}\right), 1.26\left(\mathrm{dd},{ }^{3} J=7.1 \mathrm{~Hz},{ }^{3} J=7.1 \mathrm{~Hz}, 3 \mathrm{H}, \mathrm{CH}_{2} \underline{\mathrm{CH}}_{3}\right) .{ }^{13} \mathbf{C}-\mathbf{N M R}(75 \mathrm{MHz}$, 


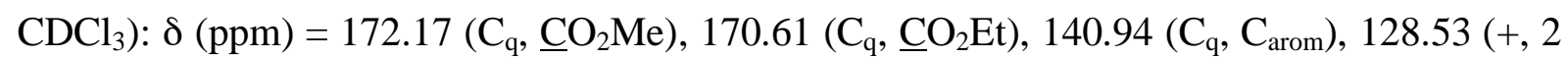
C, $\mathrm{C}_{\text {arom-meta }}$ ), 128.37 (+, 2 C, $\mathrm{C}_{\text {arom-ortho }}$ ), 126.18 (+, $\left.\mathrm{C}_{\text {arom-para }}\right), 82.24$ (+, C-3), 66.99 (+, C-1), 60.58 (-, $\left.\underline{\mathrm{CH}}_{2} \mathrm{CH}_{3}\right), 51.98\left(+, \mathrm{CO}_{2} \underline{\mathrm{CH}}_{3}\right), 42.33$ (+, C-4), 34.64 (-, $\left.\underline{\mathrm{C}} \mathrm{H}_{2} \mathrm{Ph}\right), 29.93$ (+, C-5), $29.48\left(-, \underline{\mathrm{CH}}_{2} \mathrm{CH}_{2} \mathrm{Ph}\right), 25.21$ (+, C-6), $14.24\left(+, \mathrm{CH}_{2} \mathrm{CH}_{3}\right) . \mathbf{I R}(\mathrm{KBr}): v\left(\mathrm{~cm}^{-1}\right)=3063,3027$, 2982, 2951, 2909, 2865, 1750, 1716, 1496, 1455, 1413, 1370, 1304, 1263, 1205, 1186, 1070, 1031, 990, 924, 874, 854, 749, 701. $[\alpha]_{\mathrm{D}}{ }^{20}\left(\mathrm{CH}_{2} \mathrm{Cl}_{2}, \mathrm{c}=1.6\right)=+10.8 . \mathrm{MS}(\mathrm{EI}-\mathrm{MS}): m / z(\%)$ = 318.1 (1) $\left[\mathrm{M}^{+}\right], 229.1$ (56), 213.1 (15), 185.1 (25), 129.1 (23), 104.0 (51), 91.0 (100) [PhCH$\left.{ }_{2}^{+}\right]$. HRMS (EI-MS): für $\mathrm{C}_{18} \mathrm{H}_{22} \mathrm{O}_{5}\left(\mathrm{M}^{+}\right)$calcd. 318.1467, found 318.1465.

$(2 R, 3 R, 3 a S, 6 a S)-5-0 x 0-3-p h e n y l h e x a h y d r o f u r o[2,3-b]$ furan-2-carboxylic acid (23)

Fp: $164^{\circ} \mathrm{C}$.

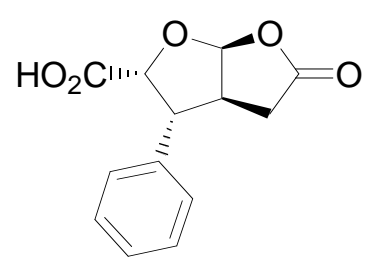

${ }^{1} \mathbf{H}-\mathbf{N M R}\left(300 \mathrm{MHz}, \mathrm{CD}_{3} \mathrm{OD}\right): \delta(\mathrm{ppm})=7.35-7.21\left(\mathrm{~m}, 5 \mathrm{H}, \mathrm{H}_{\text {arom }}\right), 6.43\left(\mathrm{~d},{ }^{3} \mathrm{~J}=5.7 \mathrm{~Hz}, 1 \mathrm{H}\right.$, H-6a), 5.05 (d, $\left.{ }^{3} J=6.4 \mathrm{~Hz}, 1 \mathrm{H}, \mathrm{H}-2\right), 4.91\left(\mathrm{~s}, \mathrm{br}, 1 \mathrm{H}, \mathrm{CO}_{2} \mathrm{H}\right), 3.63\left(\mathrm{dd},{ }^{3} J=6.4 \mathrm{~Hz},{ }^{3} J=3.5\right.$ $\mathrm{Hz}, 1 \mathrm{H}, \mathrm{H}-3$ ), 3.43 (dddd, ${ }^{3} J=10.4 \mathrm{~Hz},{ }^{3} J=5.7 \mathrm{~Hz},{ }^{3} J=3.9 \mathrm{~Hz},{ }^{3} J=3.5 \mathrm{~Hz}, 1 \mathrm{H}, \mathrm{H}-3 \mathrm{a}$ ), $3.02\left(\mathrm{dd},{ }^{2} J=18.9 \mathrm{~Hz},{ }^{3} J=10.4 \mathrm{~Hz}, 1 \mathrm{H}-4_{\text {exo }}\right), 2.70\left(\mathrm{dd},{ }^{2} J=18.9 \mathrm{~Hz},{ }^{3} J=3.9 \mathrm{~Hz}, 1 \mathrm{H}, \mathrm{H}-\right.$ $\left.4_{\text {endo }}\right) .{ }^{13}$ C-NMR $\left(75 \mathrm{MHz}, \mathrm{CD}_{3} \mathrm{OD}\right): \delta(\mathrm{ppm})=177.32\left(\mathrm{C}_{\mathrm{q}}, \mathrm{C}-5\right), 172.01\left(\mathrm{C}_{\mathrm{q}}, \mathrm{CO}_{2} \mathrm{H}\right), 139.67$ $\left(\mathrm{C}_{\mathrm{q}}, \mathrm{C}_{\mathrm{arom}}\right), 129.58$ (+, 2 C, $\left.\mathrm{C}_{\mathrm{arom}}\right), 129.31$ (+, $\left.2 \mathrm{C}, \mathrm{C}_{\mathrm{arom}}\right), 128.61$ (+, $\left.\mathrm{C}_{\mathrm{arom}}\right), 109.95$ (+, C-6a), $82.33(+, \mathrm{C}-2), 55.27$ (+, C-3), 47.56 (+, C-3a), $35.13(-, \mathrm{C}-4) . \mathbf{I R}(\mathrm{KBr}): v\left(\mathrm{~cm}^{-1}\right)=3446$, 3063, 3034, 2926, 2855, 1787, 1763, 1630, 1495, 1456, 1417, 1377, 1339, 1300, 1255, 1192, $1104,985,906,866,830,807,775,749,703,677,651,583,519 .[\alpha]_{\mathbf{D}}{ }^{20}(\mathrm{MeOH}, \mathrm{c}=0.8)=-$ 66. MS (CI-MS): $m / z(\%)=266.1(100)\left[\mathrm{M}+\mathrm{NH}_{4}{ }^{+}\right]$. HRMS (CI-MS): für $\mathrm{C}_{13} \mathrm{H}_{13} \mathrm{O}_{5}\left(\mathrm{MH}^{+}\right)$ calcd. 249.0763, found 249.0769. 
$(3 \mathrm{a} S, 4 R, 5 R, 6 \mathrm{a} S)-5$-acetyl-4-phenyltetrahydrofuro[2,3-b]furan-2(6aH)-one (29)

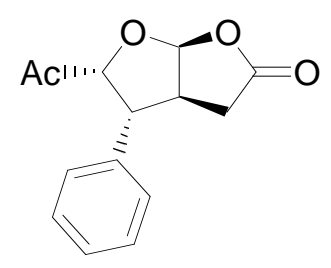

Fp: $189^{\circ} \mathrm{C}$.

${ }^{1} \mathbf{H}-\mathbf{N M R}\left(300 \mathrm{MHz}, \mathrm{CDCl}_{3}\right): \delta(\mathrm{ppm})=7.36-7.24\left(\mathrm{~m}, 3 \mathrm{H}, \mathrm{H}_{\text {arom }}\right), 7.13-7.04(\mathrm{~m}, 2 \mathrm{H}$, $\left.\mathrm{H}_{\text {arom }}\right), 6.49\left(\mathrm{~d},{ }^{3} J=5.6 \mathrm{~Hz}, 1 \mathrm{H}, \mathrm{H}-6 \mathrm{a}\right), 4.84\left(\mathrm{~d},{ }^{3} J=6.1 \mathrm{~Hz}, 1 \mathrm{H}, \mathrm{H}-2\right), 3.52\left(\mathrm{dd},{ }^{3} J=6.1 \mathrm{~Hz}\right.$, $\left.{ }^{3} J=2.6 \mathrm{~Hz}, 1 \mathrm{H}, \mathrm{H}-3\right), 3.42\left(\mathrm{dddd},{ }^{3} J=10.6 \mathrm{~Hz},{ }^{3} J=5.6 \mathrm{~Hz},{ }^{3} J=4.1 \mathrm{~Hz},{ }^{3} J=2.6 \mathrm{~Hz}, 1 \mathrm{H}, \mathrm{H}-\right.$ 3a), $3.03\left(\mathrm{dd},{ }^{2} J=18.9 \mathrm{~Hz},{ }^{3} J=10.6 \mathrm{~Hz}, 1 \mathrm{H}, \mathrm{H}-4\right.$ exo $), 2.63\left(\mathrm{dd},{ }^{2} J=18.9 \mathrm{~Hz},{ }^{3} J=4.1 \mathrm{~Hz}, 1 \mathrm{H}\right.$, H-4 $\left.4_{\text {endo }}\right), 1.72\left(\mathrm{~s}, 3 \mathrm{H}, \mathrm{CH}_{3}\right) .{ }^{13} \mathbf{C}-\mathbf{N M R}\left(75 \mathrm{MHz}, \mathrm{CDCl}_{3}\right): \delta(\mathrm{ppm})=205.54\left(\mathrm{C}_{\mathrm{q}}, \mathrm{COCH}_{3}\right)$, $174.13\left(\mathrm{C}_{\mathrm{q}}, \mathrm{C}-5\right), 137.25\left(\mathrm{C}_{\mathrm{q}}, \mathrm{C}_{\mathrm{arom}}\right), 129.04\left(+, 2 \mathrm{C}, \mathrm{C}_{\mathrm{arom}}\right), 128.07$ (+, $\left.\mathrm{C}_{\mathrm{arom}}\right), 127.86(+, 2 \mathrm{C}$, $\mathrm{C}_{\text {arom}}$ ), 107.87 (+, C-6a), 86.26 (+, C-2), 54.77 (+, C-3), 46.00 (+, C-3a), 34.50 (-, C-4), 27.83 $\left(+, \mathrm{CH}_{3}\right) . \mathbf{I R}(\mathrm{KBr}): v\left(\mathrm{~cm}^{-1}\right)=\quad 3091,3068,3038,3013,2991,2976,2944,2927,2889$, 2854, 1784, 1719, 1500, 1490, 1457, 1411, 1360, 1333, 1294, 1245, 1228, 1191, 1176, 1096, $1058,1020,994,971,920,910,881,867,834,808,798,760,718,696,641,604 .[\alpha]_{\mathrm{D}}{ }^{20}$ $\left(\mathrm{CH}_{2} \mathrm{Cl}_{2}, \mathrm{c}=0.5\right)=-5.1$. MS (EI-MS): $m / z(\%): 246.1(7)\left[\mathrm{M}^{+}\right], 218.1(31)\left[\mathrm{M}^{+} \Delta \mathrm{CO}\right], 203.1$ (86) $\left[\mathrm{M}^{+} \Delta \mathrm{COCH}_{3}\right], 157.1$ (100), 129.1 (77), 115.1 (33), 91.1 (81), 85.1 (26), 77.0 (23) $\left[\mathrm{Ph}^{+}\right]$, 57.1 (14), 43.0 (57) $\left[\mathrm{CH}_{3} \mathrm{CO}^{+}\right] . \mathbf{C}_{\mathbf{1 4}} \mathbf{H}_{\mathbf{1 4}} \mathbf{O}_{\mathbf{4}}$ : calcd. C 68.28, H 5.73, found C 68.12, H 5.67.

(2S,3R,3aS,6aS)-5-oxo-3-phenylhexahydrofuro[2,3-b]furan-2-yl acetate (30)

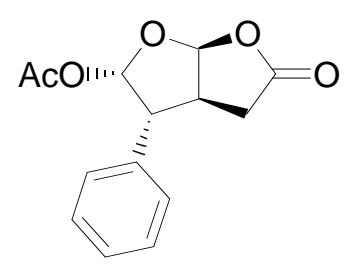

Fp: $149^{\circ} \mathrm{C}$.

${ }^{1} \mathbf{H}-\mathbf{N M R}\left(400 \mathrm{MHz}, \mathrm{CDCl}_{3}\right): \delta(\mathrm{ppm})=7.40-7.24\left(\mathrm{~m}, 5 \mathrm{H}, \mathrm{H}_{\text {arom }}\right), 6.59\left(\mathrm{~d},{ }^{3} J=4.7 \mathrm{~Hz}, 1\right.$ H, H-2), 6.22 (d, $\left.{ }^{3} J=5.4 \mathrm{~Hz}, 1 \mathrm{H}, \mathrm{H}-6 \mathrm{a}\right), 3.49$ (dddd, ${ }^{3} J=11.1 \mathrm{~Hz},{ }^{3} J=8.3 \mathrm{~Hz},{ }^{3} J=5.4 \mathrm{~Hz}$, $\left.{ }^{3} J=1.1 \mathrm{~Hz}, 1 \mathrm{H}, \mathrm{H}-3 \mathrm{a}\right), 3.32\left(\mathrm{dd},{ }^{3} J=11.1 \mathrm{~Hz},{ }^{3} J=4.7 \mathrm{~Hz}, 1 \mathrm{H}, \mathrm{H}-3\right), 2.86\left(\mathrm{dd},{ }^{2} J=18.2 \mathrm{~Hz}\right.$, $\left.{ }^{3} J=8.3 \mathrm{~Hz}, 1 \mathrm{H}, \mathrm{H}-4_{\text {exo }}\right), 2.55\left(\mathrm{dd},{ }^{2} J=18.2 \mathrm{~Hz},{ }^{3} J=1.1 \mathrm{~Hz}, 1 \mathrm{H}, \mathrm{H}-4_{\text {endo }}\right), 1.90(\mathrm{~s}, 3 \mathrm{H}$, $\left.\mathrm{CH}_{3}\right) .{ }^{13} \mathbf{C}-\mathbf{N M R}\left(75 \mathrm{MHz}, \mathrm{CDCl}_{3}\right): \delta(\mathrm{ppm})=172.99\left(\mathrm{C}_{\mathrm{q}}, \mathrm{C}-5\right), 168.89\left(\mathrm{C}_{\mathrm{q}}, \underline{\mathrm{CO}}_{2} \mathrm{CH}_{3}\right)$, $132.65\left(\mathrm{C}_{\mathrm{q}}, \mathrm{C}_{\text {arom }}\right), 128.98$ (+, $\left.2 \mathrm{C}, \mathrm{C}_{\text {arom }}\right), 128.81$ (+, $\left.2 \mathrm{C}, \mathrm{C}_{\text {arom }}\right), 128.31$ (+, $\left.\mathrm{C}_{\text {arom }}\right), 107.02(+$, 
C-6a), 98.87 (+, C-2), 53.36 (+, C-3), 42.36 (+, C-3a), 34.18 (-, C-4), $20.84\left(+, \mathrm{CH}_{3}\right)$. IR $(\mathrm{KBr}): v\left(\mathrm{~cm}^{-1}\right)=3056,3034,3016,2994,2977,2941,2926,2856,1786,1735,1498,1457$, 1432, 1381, 1352, 1322, 1293, 1260, 1235, 1160, 1112, 1092, 1030, 1008, 992, 928, 915, 900, $871,775,731,704,674,663,633,610 .[\alpha]_{\mathrm{D}}{ }^{20}\left(\mathrm{CH}_{2} \mathrm{Cl}_{2}, \mathrm{c}=1.4\right)=-90 . \mathrm{MS}(\mathrm{EI}-\mathrm{MS}): \mathrm{m} / \mathrm{z}$

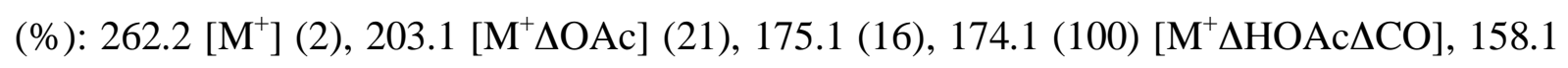
(13), 157.1 (19), 146.1 (17), 145.1 (96), 132.1 (79), 131.1 (74) [Ph-CH=CCHCO ${ }^{+}$, 129.1 (28), 117.1 (36), 91.1 (42) $\left[\mathrm{PhCH}_{2}^{+}\right], 77.1$ (12) $\left[\mathrm{Ph}^{+}\right], 43.0$ (64) $\left[\mathrm{CH}_{3} \mathrm{CO}^{+}\right]$. HRMS (EI-MS): für $\mathrm{C}_{14} \mathrm{H}_{14} \mathrm{O}_{5}\left(\mathrm{M}^{+}\right)$calcd. 262.0841, found 262.0838.

(2R,3S,3aR,6aR)-5-oxo-3-phenethylhexahydrofuro[2,3-b]furan-2-carboxylic acid (31)

Fp: $146^{\circ} \mathrm{C}$.

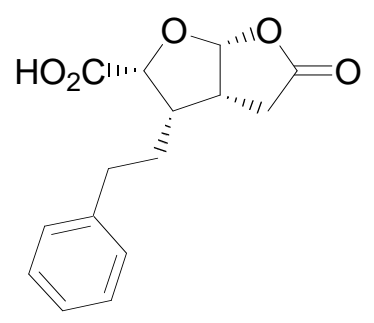

${ }^{1} \mathbf{H}-\mathbf{N M R}\left(400 \mathrm{MHz}, \mathrm{CD}_{3} \mathrm{OD}\right): \delta(\mathrm{ppm})=7.30-7.15\left(\mathrm{~m}, 5 \mathrm{H}, \mathrm{H}_{\text {arom }}\right), 6.12\left(\mathrm{~d},{ }^{3} J=6.0 \mathrm{~Hz}, 1\right.$ H, H-6a), 4.90 (s, br, $\left.1 \mathrm{H}, \mathrm{CO}_{2} \mathrm{H}\right), 4.62\left(\mathrm{~d},{ }^{3} J=8.8 \mathrm{~Hz}, 1 \mathrm{H}, \mathrm{H}-2\right), 3.29$ (dddd, ${ }^{3} J=11.5 \mathrm{~Hz},{ }^{3} J$ $\left.=8.4 \mathrm{~Hz},{ }^{3} J=6.5 \mathrm{~Hz},{ }^{3} J=6.0 \mathrm{~Hz}, 1 \mathrm{H}, \mathrm{H}-3 \mathrm{a}\right), 2.79-2.65\left(\mathrm{~m}, 2 \mathrm{H}, \underline{\mathrm{H}}_{2} \mathrm{Ph}\right), 2.78\left(\mathrm{dd},{ }^{3} J=8.8\right.$ $\left.\mathrm{Hz},{ }^{3} J=8.4 \mathrm{~Hz}, 1 \mathrm{H}, \mathrm{H}-3\right), 2.56\left(\mathrm{dd},{ }^{2} J=18.8 \mathrm{~Hz},{ }^{3} J=6.5 \mathrm{~Hz}, 1 \mathrm{H}, \mathrm{H}-4_{\text {endo }}\right), 2.45\left(\mathrm{dd},{ }^{2} J=\right.$ $\left.18.8 \mathrm{~Hz},{ }^{3} J=11.5 \mathrm{~Hz}, 1 \mathrm{H}, \mathrm{H}-4_{\text {exo }}\right), 1.96-1.90$ (m, $\left.1 \mathrm{H}, \mathrm{C}_{\mathbf{H H C H}} \mathrm{Ph}\right), 1.76-1.70$ (m, $1 \mathrm{H}$, $\left.\mathrm{CH}_{\underline{H C H}} \mathrm{Ph}\right) .{ }^{13} \mathrm{C}-\mathrm{NMR}\left(75 \mathrm{MHz}, \mathrm{CD}_{3} \mathrm{OD}\right): \delta(\mathrm{ppm})=179.28\left(\mathrm{C}_{\mathrm{q}}, \mathrm{C}-5\right), 174.62\left(\mathrm{C}_{\mathrm{q}}, \mathrm{CO}_{2} \mathrm{H}\right)$, $142.81\left(\mathrm{C}_{\mathrm{q}}, \mathrm{C}_{\text {arom }}\right), 129.61$ (+, $\left.2 \mathrm{C}, \mathrm{C}_{\text {arom }}\right), 129.46$ (+, $\left.2 \mathrm{C}, \mathrm{C}_{\text {arom }}\right), 127.24$ (+, $\left.\mathrm{C}_{\text {arom }}\right), 110.68$ (+, C-6a), 82.76 (+, C-2), 44.58 (+, C-3), 42.33 (+, C-3a), 35.36 (-, $\left.\mathrm{CH}_{2} \mathrm{Ph}\right), 29.87$ (-, $\left.\mathrm{CH}_{2} \mathrm{CH}_{2} \mathrm{Ph}\right), 29.43(-, \mathrm{C}-4)$. IR $(\mathrm{KBr}): v\left(\mathrm{~cm}^{-1}\right)=3422,3022,2926,2857,1773,1385,1202$, $1111,1088,1017,993,975,751,737,700,627 .[\alpha]_{\mathbf{D}}{ }^{20}(\mathrm{MeOH}, \mathrm{c}=0.6)=-47 . \mathbf{M S}(\mathrm{EI}-\mathrm{MS})$ : $m / z(\%)=258.1(2)\left[\mathrm{M}^{+} \Delta \mathrm{H}_{2} \mathrm{O}\right], 230.1$ (11) $\left[\mathrm{M}^{+} \Delta \mathrm{H}_{2} \mathrm{O} \Delta \mathrm{CO}\right], 201.1$ (37), 129.1 (27), 105.1 (14) $\left[\mathrm{Ph}\left(\mathrm{CH}_{2}\right)_{2}{ }^{+}\right], 104.1$ (66) $\left[\mathrm{PhCH}=\mathrm{CH}_{2}{ }^{+}\right], 91.1$ (100) $\left[\mathrm{PhCH}_{2}^{+}\right] . \mathbf{C}_{\mathbf{1 5}} \mathbf{H}_{16} \mathbf{O}_{5}$ : calcd. C 65.21, H 5.84, found C 65.13, H 5.84. 
$(2 R, 3 R, 3 a S, 6 a S)-3-($ naphthalen-2-yl)-5-oxohexahydrofuro[2,3-b]furan-2-carboxylic acid (32)

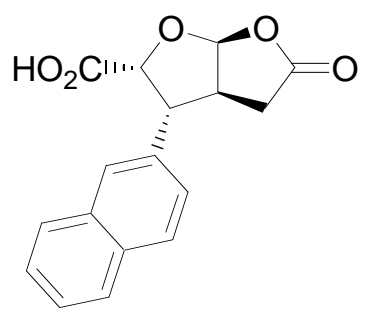

${ }^{1}$ H-NMR (400 MHz, CD 3 OD): $\delta(\mathrm{ppm})=7.84-7.76\left(\mathrm{~m}, 3 \mathrm{H}, \mathrm{H}_{\text {arom }}\right), 7.72\left(\mathrm{~m}, 1 \mathrm{H}, \mathrm{H}_{\text {arom }}\right)$, $7.48-7.40$ (m, $\left.2 \mathrm{H}, \mathrm{H}_{\text {arom }}\right), 7.38-7.35\left(\mathrm{~m}, 1 \mathrm{H}, \mathrm{H}_{\text {arom }}\right), 6.52\left(\mathrm{~d},{ }^{3} \mathrm{~J}=5.6 \mathrm{~Hz}, 1 \mathrm{H}, \mathrm{H}-6 \mathrm{a}\right), 5.13$ $\left(\mathrm{d},{ }^{3} J=6.5 \mathrm{~Hz}, 1 \mathrm{H}, \mathrm{H}-2\right), 4.84\left(\mathrm{~s}, \mathrm{br}, 1 \mathrm{H}, \mathrm{CO}_{2} \mathrm{H}\right), 3.82\left(\mathrm{dd},{ }^{3} \mathrm{~J}=6.5 \mathrm{~Hz},{ }^{3} J=3.7 \mathrm{~Hz}, 1 \mathrm{H}, \mathrm{H}-\right.$ 3), 3.57 (dddd, ${ }^{3} J=10.3 \mathrm{~Hz},{ }^{3} J=5.6 \mathrm{~Hz},{ }^{3} J=3.8 \mathrm{~Hz},{ }^{3} J=3.7 \mathrm{~Hz}, 1 \mathrm{H}, \mathrm{H}-3 \mathrm{a}$ ), 3.07 (dd, ${ }^{2} J=$ $\left.18.8 \mathrm{~Hz},{ }^{3} \mathrm{~J}=10.3 \mathrm{~Hz}, 1 \mathrm{H}-4_{\text {exo }}\right), 2.77\left(\mathrm{dd},{ }^{2} \mathrm{~J}=18.8 \mathrm{~Hz},{ }^{3} \mathrm{~J}=3.8 \mathrm{~Hz}, 1 \mathrm{H}, \mathrm{H}-4_{\text {endo }}\right) .{ }^{\mathbf{1 3}} \mathbf{C}-\mathbf{N M R}$ $\left(75 \mathrm{MHz}, \mathrm{CD}_{3} \mathrm{OD}\right): \delta(\mathrm{ppm})=177.30\left(\mathrm{C}_{\mathrm{q}}, \mathrm{C}-5\right), 172.12\left(\mathrm{C}_{\mathrm{q}}, \mathrm{CO}_{2} \mathrm{H}\right), 137.11\left(\mathrm{C}_{\mathrm{q}}, \mathrm{C}_{\text {arom }}\right)$, $134.77\left(\mathrm{C}_{\mathrm{q}}, \mathrm{C}_{\text {arom }}\right), 134.26\left(\mathrm{C}_{\mathrm{q}}, \mathrm{C}_{\text {arom }}\right), 129.21\left(+, \mathrm{CH}_{\text {arom }}\right), 128.96\left(+, \mathrm{CH}_{\text {arom }}\right), 128.58(+$, $\mathrm{CH}_{\text {arom }}$ ), 128.09 (+, $\left.\mathrm{CH}_{\text {arom }}\right), 127.37$ (+, $\left.\mathrm{CH}_{\text {arom }}\right), 127.28$ (+, $\left.\mathrm{CH}_{\text {arom }}\right), 127.10$ (+, $\left.\mathrm{CH}_{\text {arom }}\right)$, 110.04 (+, C-6a), 82.46 (+, C-2), 55.39 (+, C-3), 47.53 (+, C-3a), 35.20 (-, C-4). IR (KBr): v $\left(\mathrm{cm}^{-1}\right)=3233,3053,3018,2936,1757,1373,1317,1252,1195,1099,1024,996,984,901$, 870, 813, 752, 708, 632. MS (EI-MS): $m / z(\%)=298.1(100)\left[\mathrm{M}^{+}\right], 252.1$ (15) [M $\mathrm{M}^{+} \Delta \mathrm{H}_{2} \mathrm{O} \Delta \mathrm{CO}$ ], 224.1 (15) $\left[\mathrm{M}^{+} \Delta \mathrm{H}_{2} \mathrm{O} \Delta 2 \mathrm{CO}\right], 207.1$ (21), 195.1 (42), 182.1 (51), 181.1 (44), 165.1 (45), 152.1 (35), 141.1 (32), 128.1 (18), 127.1 (7) $\left[\mathrm{C}_{10} \mathrm{H}_{7}^{+}\right], 115.1$ (13). HRMS (EIMS): für $\mathrm{C}_{17} \mathrm{H}_{14} \mathrm{O}_{5}\left(\mathrm{M}^{+}\right)$: calcd. 298.0841, found 298.0839.

$(2 R / S, 3 S, 3 a R, 6 a R)-5-0 x 0-3-p h e n e t h y l h e x a h y d r o f u r o[2,3-b]$ furan-2-yl acetate (33)

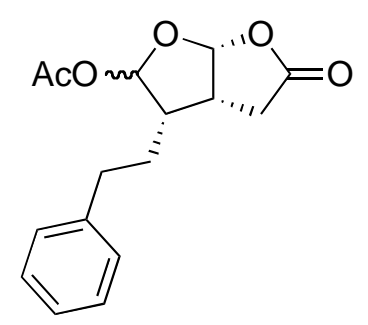

NMR-data for the $2 R$-Isomer:

${ }^{1} \mathbf{H}-\mathbf{N M R}\left(600 \mathrm{MHz}, \mathrm{CDCl}_{3}\right): \delta(\mathrm{ppm})=7.34-7.30\left(\mathrm{~m}, 2 \mathrm{H}, \mathrm{H}_{\text {arom-ortho }}\right)$ 7.26-7.22 (m, $1 \mathrm{H}$, $\mathrm{H}_{\text {arom-para }}$ ), 7.20-7.16 (m, $\left.2 \mathrm{H}, \mathrm{H}_{\text {arom-meta }}\right), 6.21$ (d, $\left.{ }^{3} J=5.0 \mathrm{~Hz}, 1 \mathrm{H}, \mathrm{H}-2\right), 6.09$ (d, ${ }^{3} J=5.4 \mathrm{~Hz}$, $1 \mathrm{H}, \mathrm{H}-6 \mathrm{a}$ ), 3.21 (dddd, ${ }^{3} J=9.1 \mathrm{~Hz},{ }^{3} J=8.1 \mathrm{~Hz},{ }^{3} J=7.0 \mathrm{~Hz},{ }^{3} J=5.4 \mathrm{~Hz}, 1 \mathrm{H}, \mathrm{H}-3 \mathrm{a}$ ), 2.76 $\left(\mathrm{ddd},{ }^{2} J=14.3 \mathrm{~Hz},{ }^{3} J=8.7 \mathrm{~Hz},{ }^{3} J=6.0 \mathrm{~Hz}, 1 \mathrm{H}, \mathrm{CHHPh}\right), 2.64\left(\mathrm{ddd},{ }^{2} J=14.3 \mathrm{~Hz},,^{3} J=8.3\right.$ 
$\left.\mathrm{Hz},{ }^{3} J=7.1 \mathrm{~Hz}, 1 \mathrm{H}, \mathrm{C} \underline{\mathrm{H} H P h}\right), 2.55\left(\mathrm{dd},{ }^{2} J=18.4 \mathrm{~Hz},{ }^{3} J=7.0 \mathrm{~Hz}, 1 \mathrm{H}, \mathrm{H}-4\right), 2.52$ (dd, ${ }^{2} J=$ $\left.18.4 \mathrm{~Hz},{ }^{3} J=9.1 \mathrm{~Hz}, 1 \mathrm{H}, \mathrm{H}-4\right), 2.42\left(\mathrm{dddd},{ }^{3} J=8.1 \mathrm{~Hz},{ }^{3} J=8.1 \mathrm{~Hz},{ }^{3} J=7.3 \mathrm{~Hz},{ }^{3} J=5.0 \mathrm{~Hz}\right.$, $1 \mathrm{H}, \mathrm{H}-3), 2.12\left(\mathrm{~s}, 3 \mathrm{H}, \mathrm{CH}_{3}\right), 1.86\left(\mathrm{dddd},{ }^{2} J=14.0 \mathrm{~Hz},{ }^{3} J=8.3 \mathrm{~Hz},{ }^{3} J=8.1 \mathrm{~Hz},{ }^{3} J=6.0 \mathrm{~Hz}\right.$, $1 \mathrm{H}, \mathrm{CHHCH}_{2} \mathrm{Ph}$ ), $1.76\left(\mathrm{dddd},{ }^{2} J=14.0 \mathrm{~Hz},{ }^{3} J=8.7 \mathrm{~Hz},{ }^{3} J=7.3 \mathrm{~Hz},{ }^{3} J=7.1 \mathrm{~Hz}, 1 \mathrm{H},-\right.$ $\left.\mathrm{CH}_{\underline{H}} \mathrm{CH}_{2} \mathrm{Ph}\right) .{ }^{13} \mathrm{C}-\mathrm{NMR}\left(75 \mathrm{MHz}, \mathrm{CDCl}_{3}\right): \delta(\mathrm{ppm})=174.61\left(\mathrm{C}_{\mathrm{q}}, \mathrm{C}-5\right), 169.74\left(\mathrm{C}_{\mathrm{q}}, \underline{\mathrm{COCH}}_{3}\right)$, $140.42\left(\mathrm{C}_{\mathrm{q}}, \mathrm{C}_{\text {arom }}\right), 128.69$ (+, $\left.2 \mathrm{C}, \mathrm{CH}_{\text {arom }}\right), 128.37$ (+, $\left.2 \mathrm{C}, \mathrm{CH}_{\text {arom}}\right), 126.49$ (+, $\mathrm{C}_{\text {arom-para }}$ ), 106.33 (+, C-6a), 100.84 (+, C-2), 44.75 (+, C-3), 40.44 (+, C-3a), $34.12\left(-, \underline{C H}_{2} \mathrm{Ph}\right), 28.71$ (-, $\left.\mathrm{CH}_{2} \mathrm{CH}_{2} \mathrm{Ph}\right), 28.51$ (-, C-4), $21.09\left(+, \mathrm{CH}_{3}\right)$.

NMR-data for the $2 S$-Isomer:

${ }^{1} \mathbf{H}-\mathbf{N M R}\left(600 \mathrm{MHz}, \mathrm{CDCl}_{3}\right): \delta(\mathrm{ppm})=7.34$ - 7.30 (m, $\left.2 \mathrm{H}, \mathrm{H}_{\text {arom-ortho }}\right), 7.26$ - $7.22(\mathrm{~m}, 1 \mathrm{H}$, $\mathrm{H}_{\text {arom-para }}$ ), 7.20 - 7.16 (m, $2 \mathrm{H}, \mathrm{H}_{\text {arom-meta }}$ ), 6.28 (d, $\left.{ }^{3} J=4.4 \mathrm{~Hz}, 1 \mathrm{H}, \mathrm{H}-2\right), 6.11$ (d, ${ }^{3} J=6.1 \mathrm{~Hz}$, $1 \mathrm{H}, \mathrm{H}-6 \mathrm{a}$ ), 3.17 (dddd, ${ }^{3} J=11.4 \mathrm{~Hz},{ }^{3} J=8.6 \mathrm{~Hz},{ }^{3} J=6.1 \mathrm{~Hz},{ }^{3} J=5.1 \mathrm{~Hz} 1 \mathrm{H}, \mathrm{H}-3 \mathrm{a}$ ), 2.78 $2.73(\mathrm{~m}, 1 \mathrm{H}, \mathrm{C} \underline{H} H P h), 2.76\left(\mathrm{dd},{ }^{2} J=18.4 \mathrm{~Hz},{ }^{3} J=5.1 \mathrm{~Hz}, 1 \mathrm{H}, \mathrm{H}-4\right), 2.69-2.65$ (m, $1 \mathrm{H}$, CHㅂPh), 2.52 (dd, $\left.{ }^{2} J=18.4 \mathrm{~Hz},{ }^{3} J=11.4 \mathrm{~Hz}, 1 \mathrm{H}, \mathrm{H}-4\right), 2.46-2.40$ (m, $\left.1 \mathrm{H}, \mathrm{H}-3\right), 2.05$ (s, 3 $\left.\mathrm{H}, \mathrm{CH}_{3}\right), 1.96-1.90\left(\mathrm{~m}, 1 \mathrm{H}, \mathrm{C}_{\mathbf{H} H C H} \mathrm{Ph}\right), 1.84-1.76$ (m, $\left.1 \mathrm{H}, \mathrm{CH}^{\mathrm{HCH}} \mathrm{H}_{2} \mathrm{Ph}\right) .{ }^{13} \mathbf{C}-\mathbf{N M R}(75$ $\left.\mathrm{MHz}, \mathrm{CDCl}_{3}\right): \delta(\mathrm{ppm})=175.37\left(\mathrm{C}_{\mathrm{q}}, \mathrm{C}-5\right), 169.49\left(\mathrm{C}_{\mathrm{q}}, \underline{\mathrm{COCH}_{3}}\right), 140.59\left(\mathrm{C}_{\mathrm{q}}, \mathrm{C}_{\mathrm{arom}}\right), 128.74$ (+, $\left.2 \mathrm{C}, \mathrm{CH}_{\text {arom }}\right), 128.28$ (+, $\left.2 \mathrm{C}, \mathrm{CH}_{\text {arom }}\right), 126.54$ (+, $\left.\mathrm{C}_{\text {arom-para }}\right), 107.86$ (+, C-6a), 98.00 (+, C2), 44.32 (+, C-3), 38.96 (+, C-3a), 33.96 (-, $\left.\underline{\mathrm{C}} \mathrm{H}_{2} \mathrm{Ph}\right), 28.86$ (-, C-4), 26.83 (-, $\left.\underline{\mathrm{CH}}_{2} \mathrm{CH}_{2} \mathrm{Ph}\right)$, $21.17\left(+, \mathrm{CH}_{3}\right)$.

MS (PI-EI-MS): $m / z(\%): 231.0$ (3) [M $\left[\mathrm{M}^{+} \Delta \mathrm{OAc}\right] 230.0$ (9) $\left[\mathrm{M}^{+} \Delta \mathrm{HOAc}\right], 213.0$ (13), 129.0 (19), 128.0 (37), 104.0 (100) $\left[\mathrm{PhCH}=\mathrm{CH}_{2}^{+}\right], 91.0$ (59) $\left[\mathrm{PhCH}_{2}^{+}\right]$. HRMS (LSI-MS): für $\mathrm{C}_{16} \mathrm{H}_{19} \mathrm{O}_{5}\left(\mathrm{MH}^{+}\right)$calcd. 291.1232, found 291.1232. 
(1S,5S,6S)-(-)-2-Oxa-bicyclo[3.1.0]hex-3-en-3,6-dicarbonsäure-6-ethylester-3methylester

${ }^{1}$ H-NMR (300 MHz, $\mathrm{CDCl}_{3}$ )

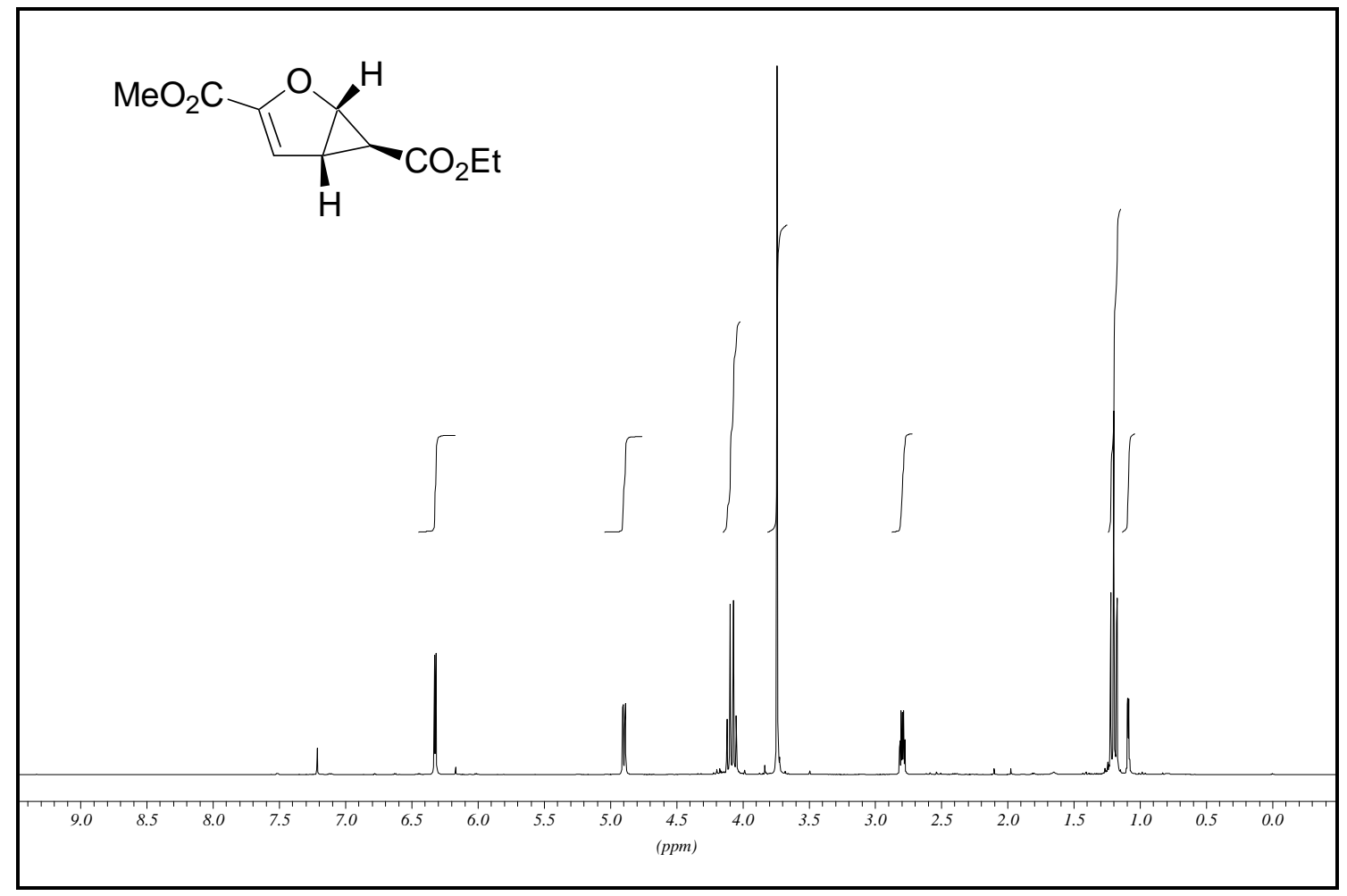

${ }^{13} \mathrm{C}-\mathrm{NMR}$ (75.5 MHz, $\mathrm{CDCl}_{3}$ )

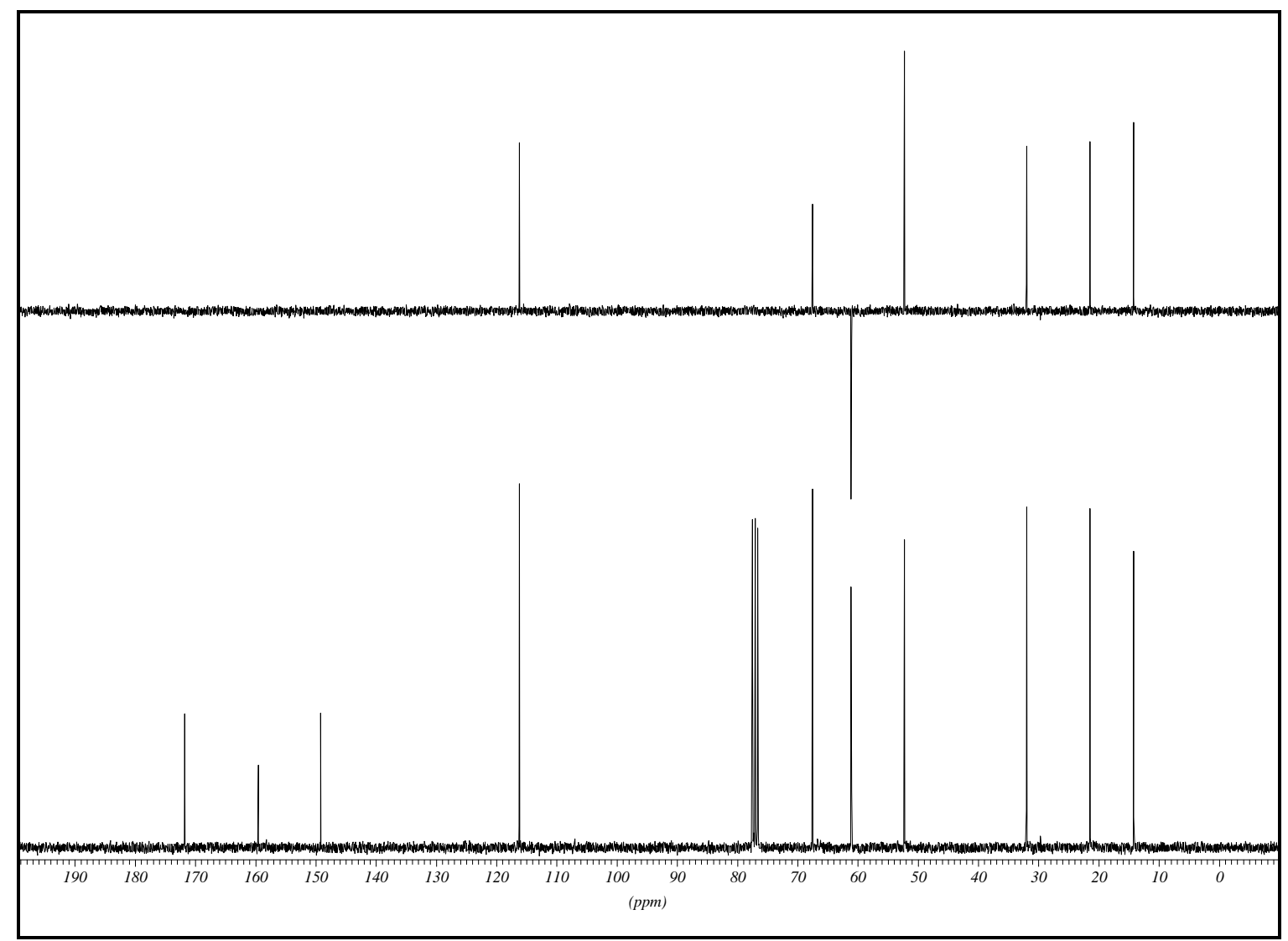


(1S,3R,5S,6S)-(+)-2-Oxa-bicyclo[3.1.0]hexan-3,6-dicarbonsäure-6-ethylester-3methylester

${ }^{1} \mathrm{H}-\mathrm{NMR}\left(300 \mathrm{MHz}, \mathrm{CDCl}_{3}\right)$

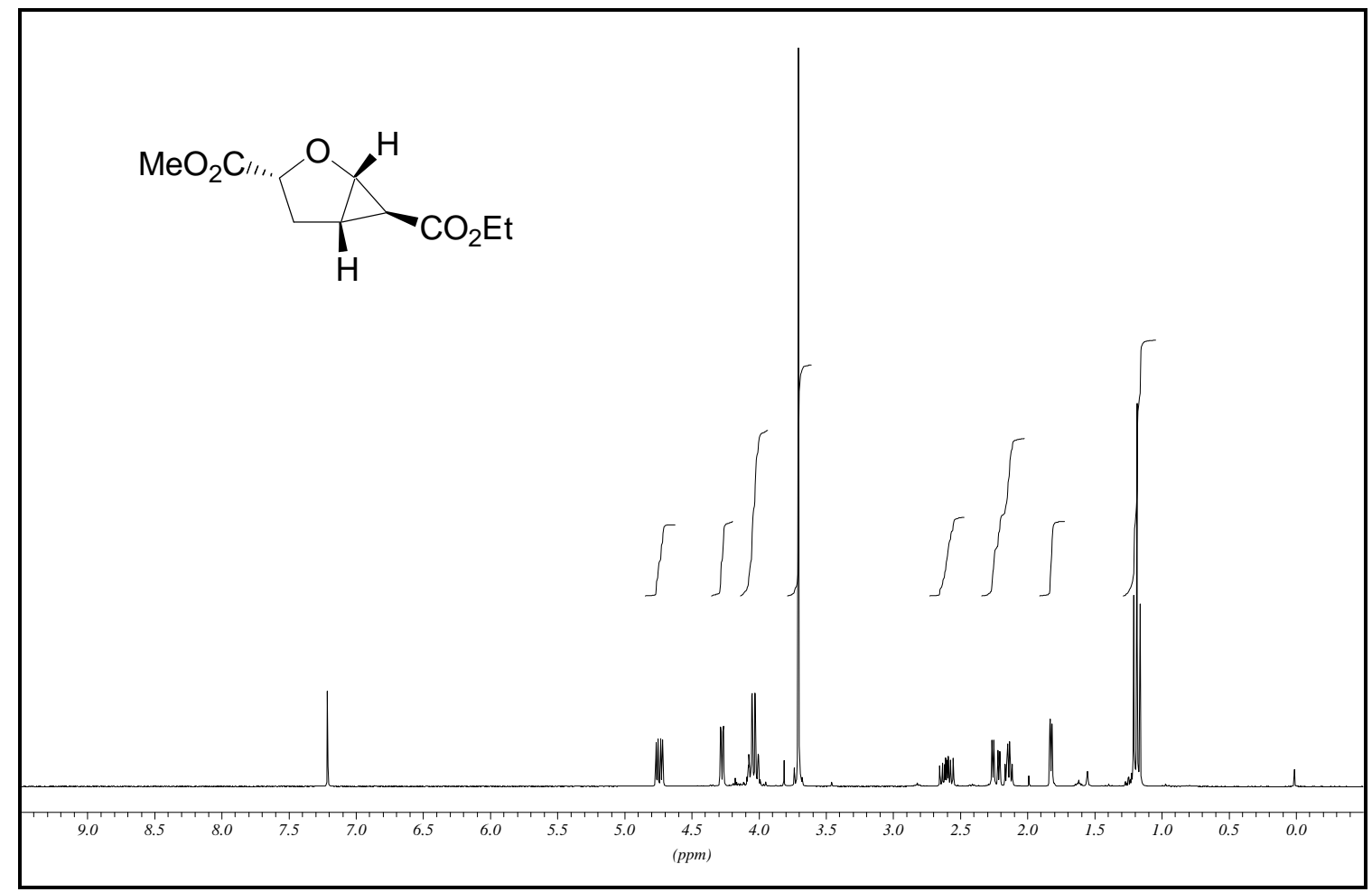

${ }^{13} \mathrm{C}-\mathrm{NMR}\left(75.5 \mathrm{MHz}, \mathrm{CDCl}_{3}\right)$

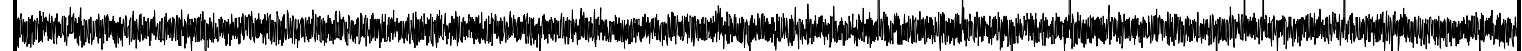

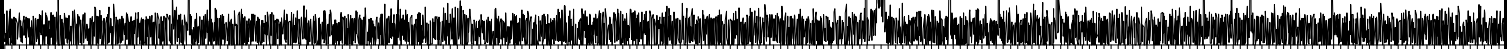
$110 \quad 100$
$(\mathrm{ppm})$ 
(2R,3aS,6aR)-(-)-5-Oxo-hexahydrofuro[2,3-b]furan-2-carbonsäure

${ }^{1}$ H-NMR (400 MHz, DMSO-d 6 )

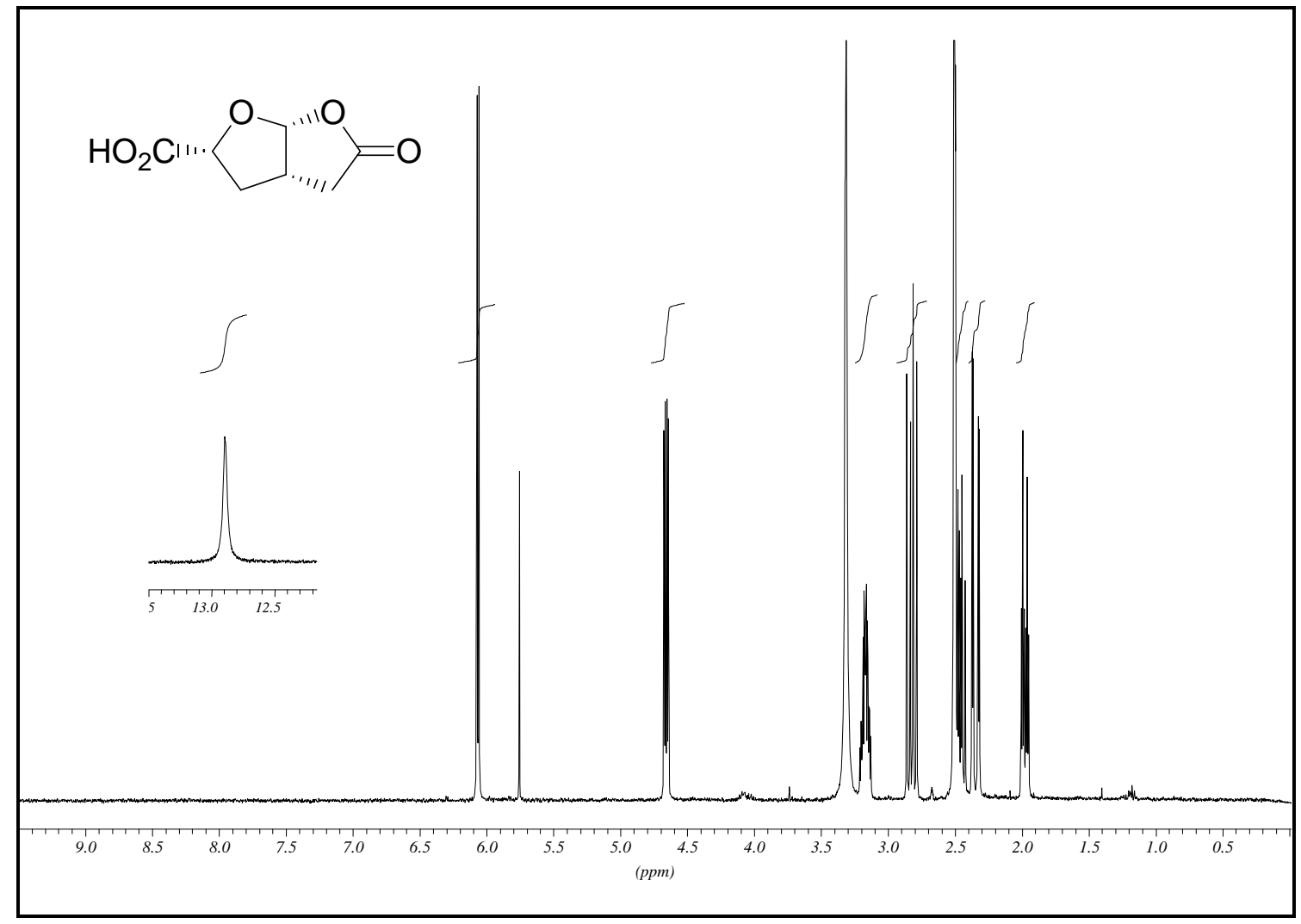

${ }^{13}$ C-NMR (100.6 MHz, DMSO- $d_{6}$ )

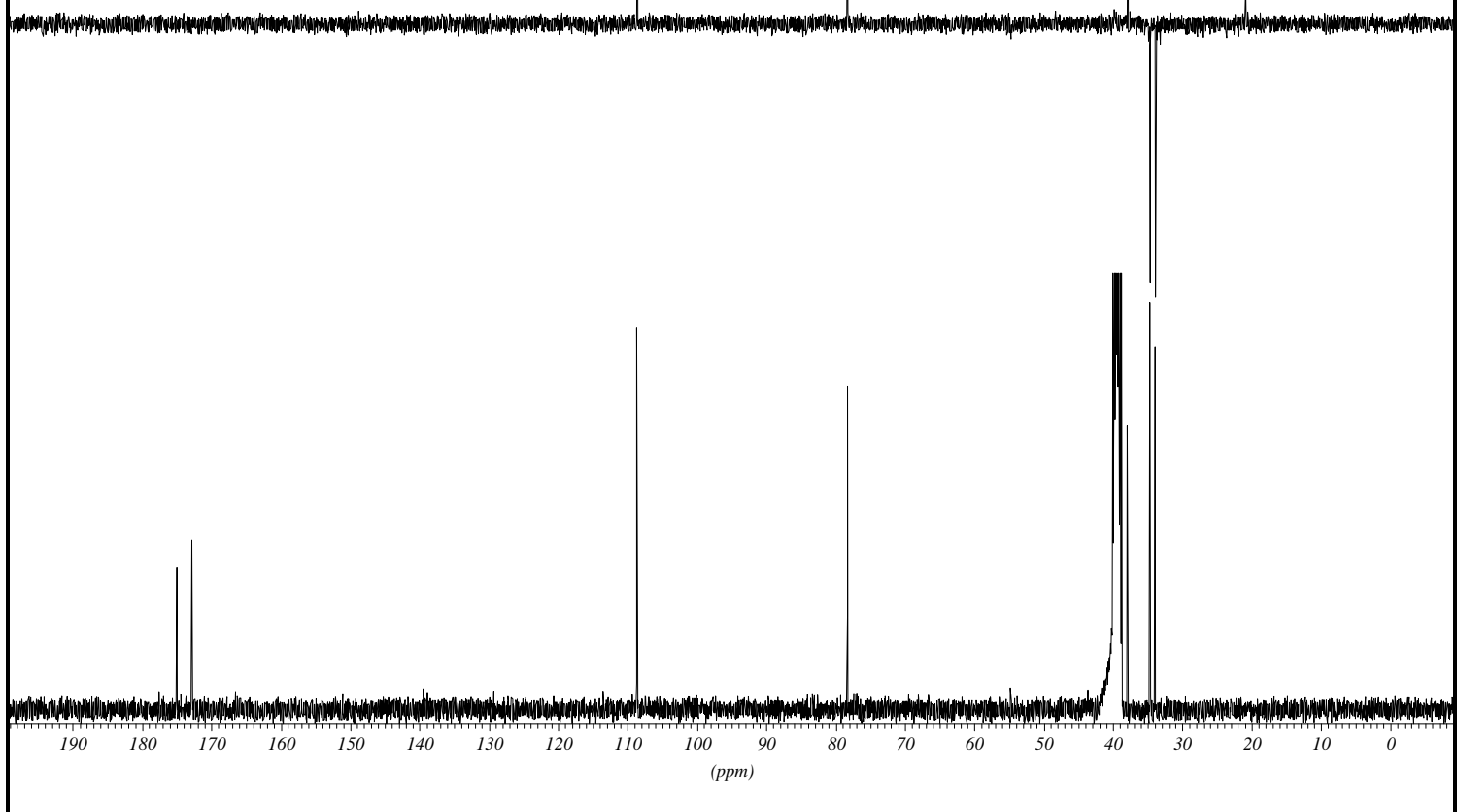


(2S,3aS,6aR)-(-)-Essigsäure-5-oxo-hexahydrofuro[2,3-b]furan-2-yl-ester

${ }^{1} \mathrm{H}-\mathrm{NMR}\left(300 \mathrm{MHz}, \mathrm{CDCl}_{3}\right)$

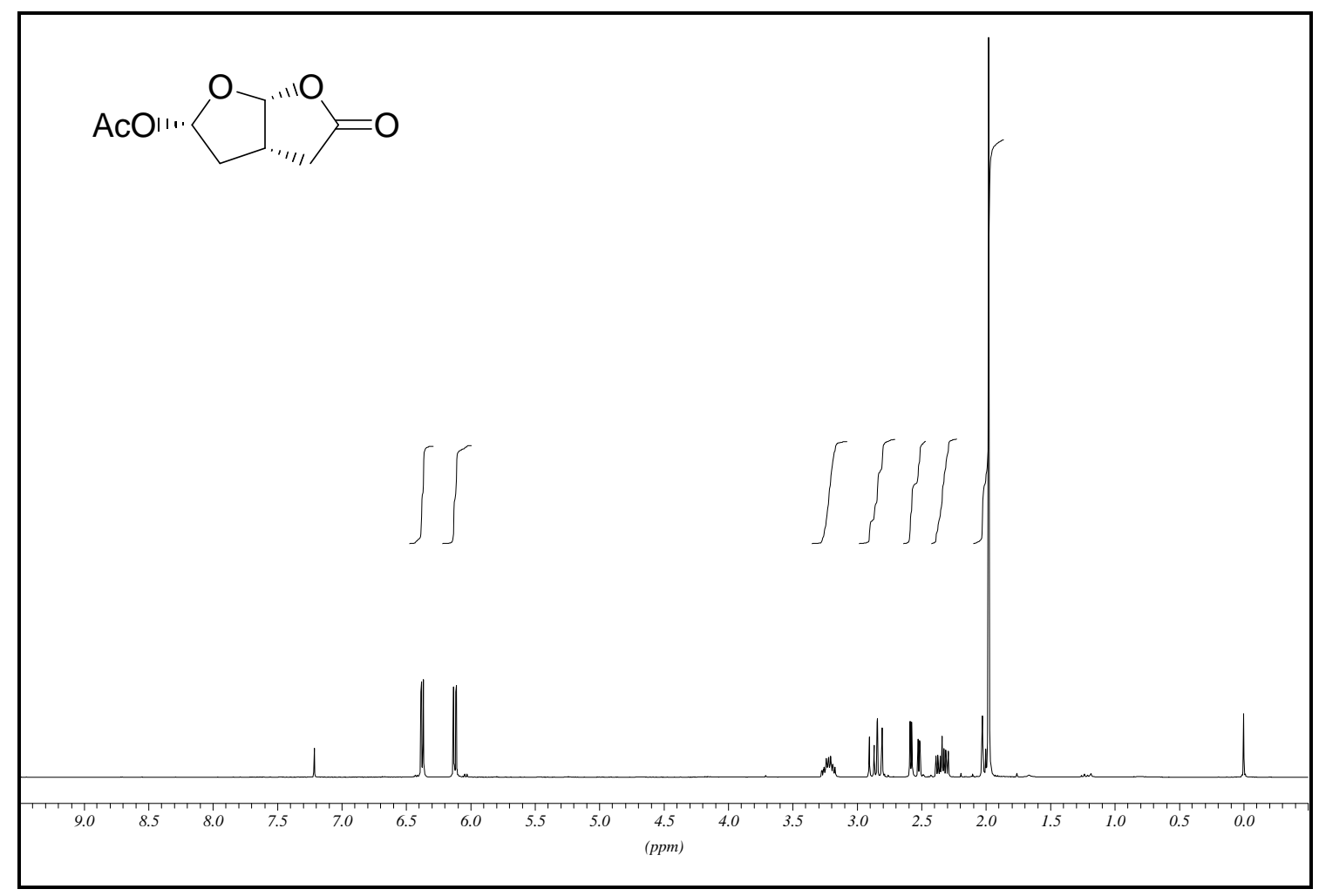

${ }^{13} \mathrm{C}-\mathrm{NMR}\left(75 \mathrm{MHz}, \mathrm{CDCl}_{3}\right)$

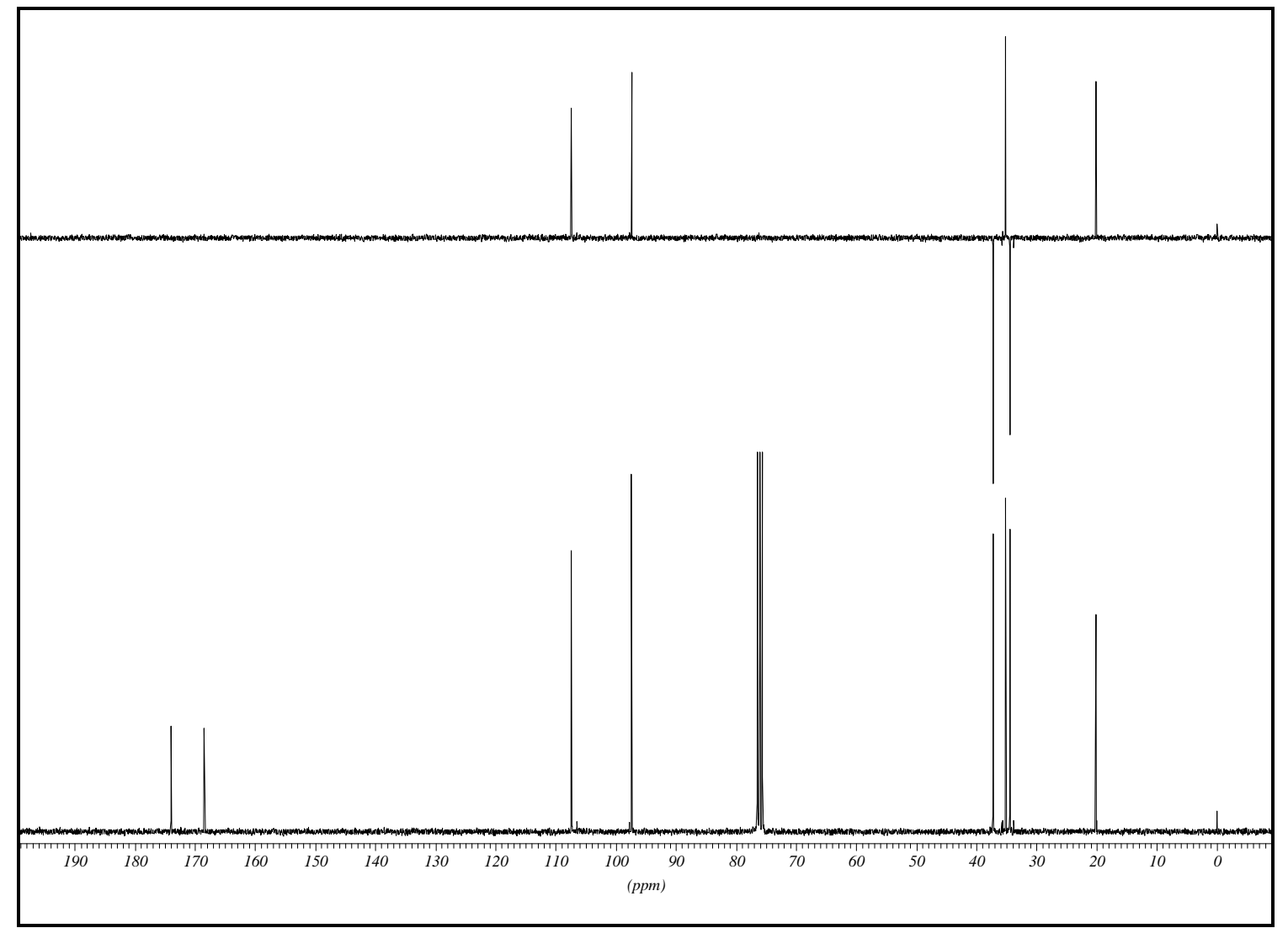


$(1 R, 3 S, 4 S, 5 S, 6 S)-(-)-3,4-D i b r o m-2-0 x a-b i c y c l o[3.1 .0]$ hexan-3,6-dicarbonsäure-6ethylester-3-methylester

${ }^{1}$ H-NMR (300 MHz, $\mathrm{CDCl}_{3}$ )

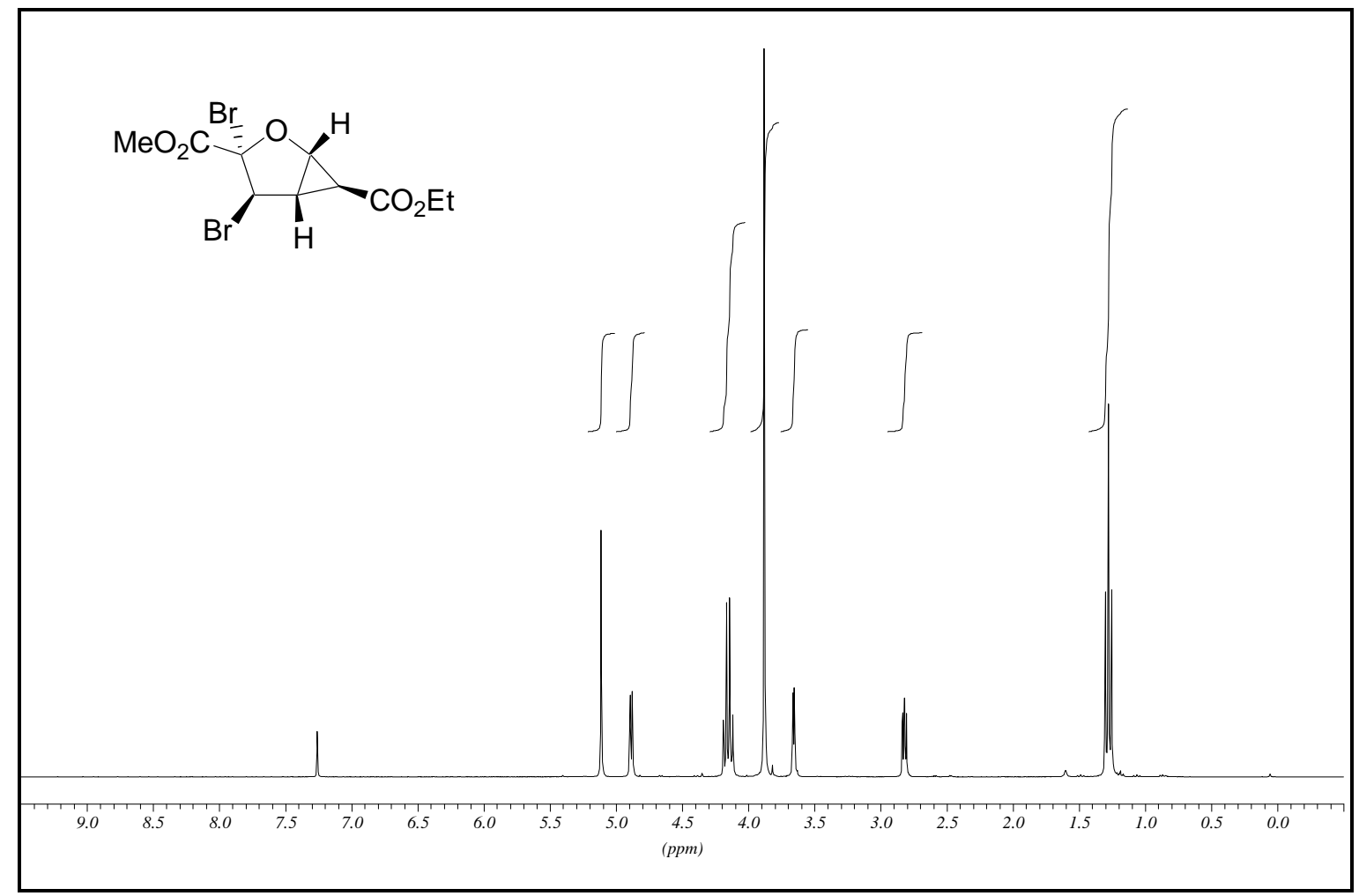

${ }^{13} \mathrm{C}$-NMR (75.5 MHz, $\mathrm{CDCl}_{3}$ )

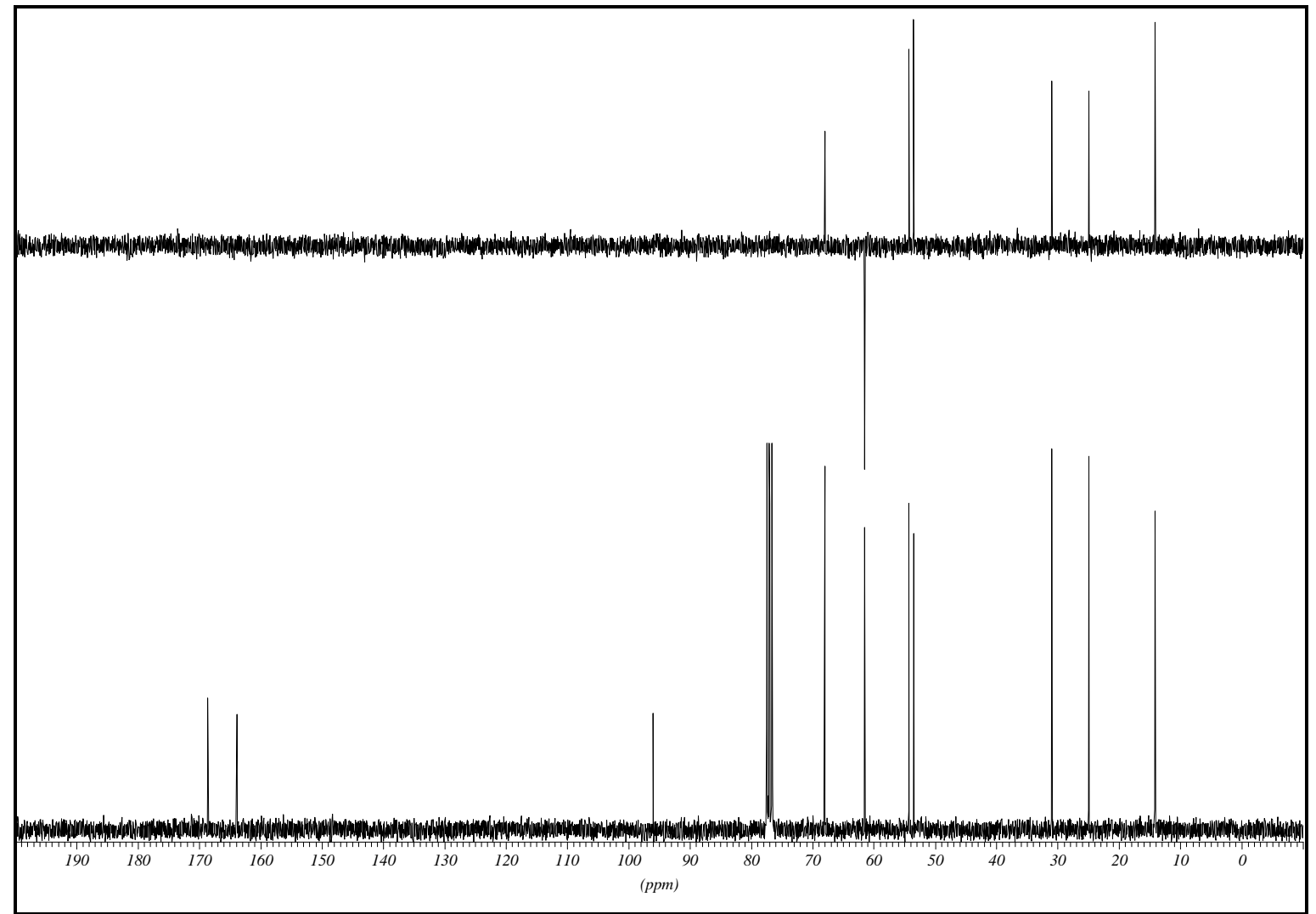


(1R,5S,6S)-(-)-4-Brom-2-oxa-bicyclo[3.1.0] hex-3-en-3,6-dicarbonsäure-6-ethylester-3methylester

${ }^{1} \mathrm{H}-\mathrm{NMR}\left(300 \mathrm{MHz}, \mathrm{CDCl}_{3}\right)$

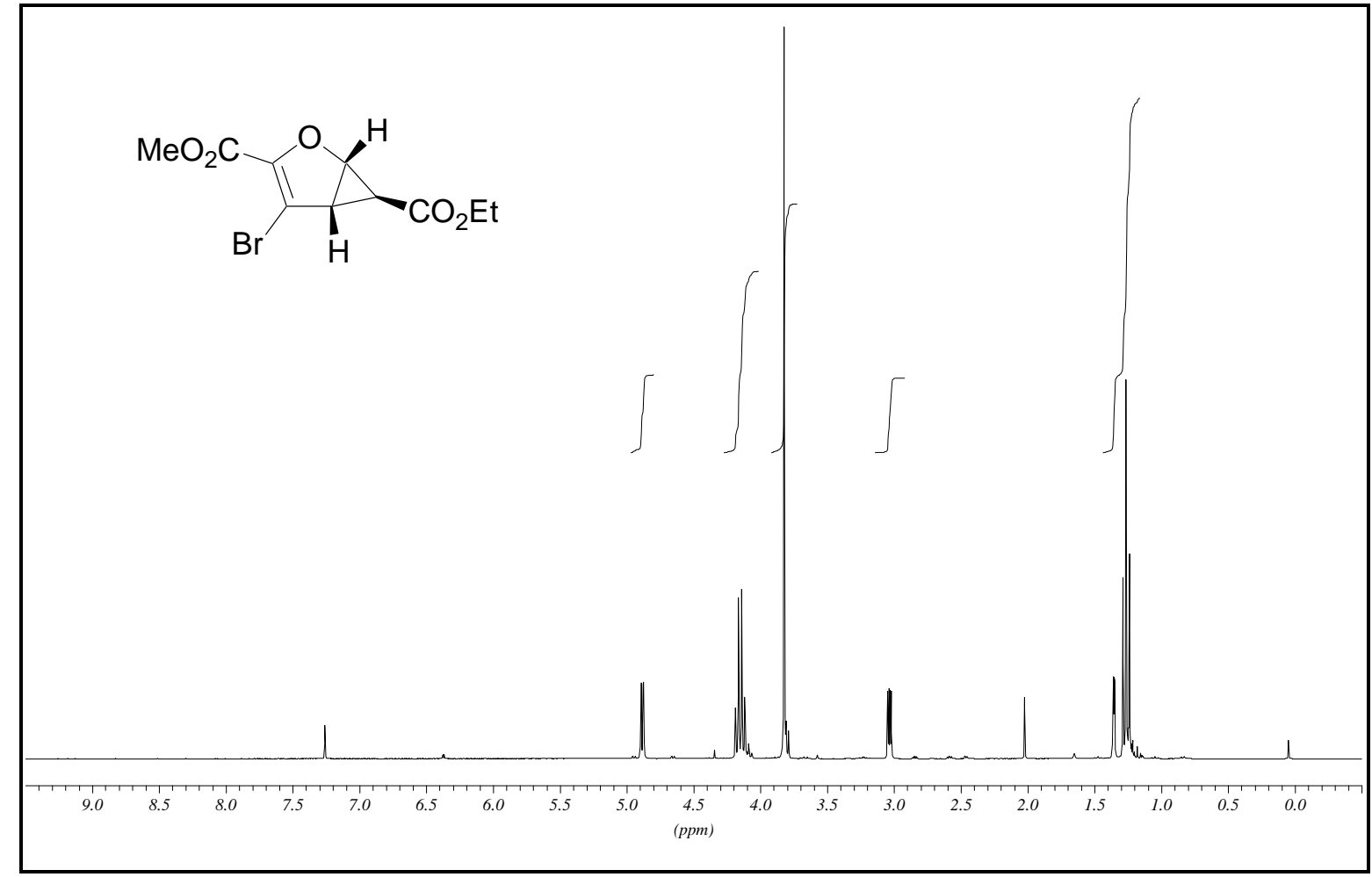

\section{${ }^{13} \mathrm{C}-\mathrm{NMR}\left(75.5 \mathrm{MHz} \mathrm{CDCl}_{3}\right)$}

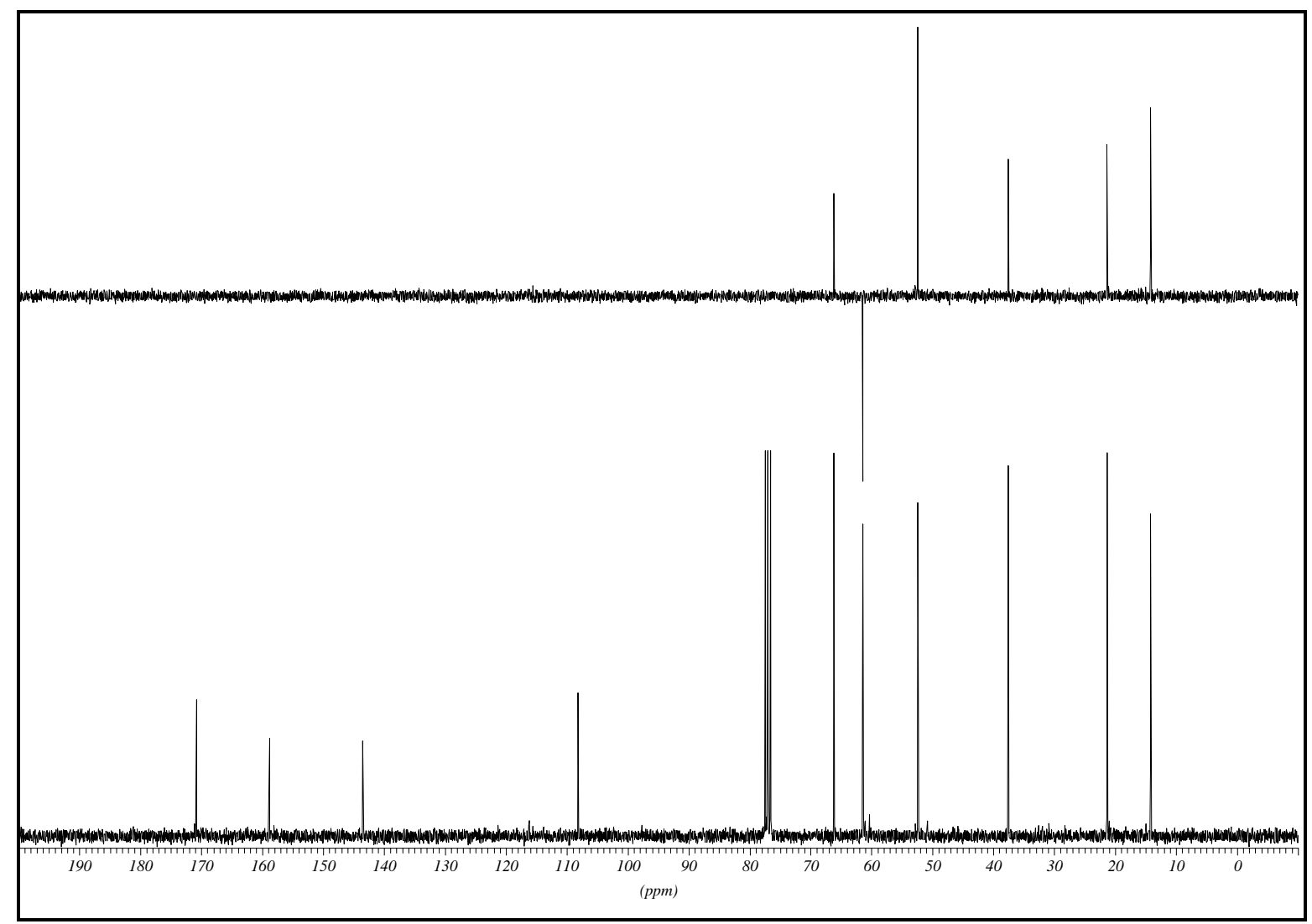


(1S,5R,6S)-(-)-4-Phenyl-2-oxa-bicyclo[3.1.0]hex-3-en-3,6-dicarbonsäure-6-ethylester-3methylester

${ }^{1} \mathrm{H}-\mathrm{NMR}\left(300 \mathrm{MHz}, \mathrm{CDCl}_{3}\right)$

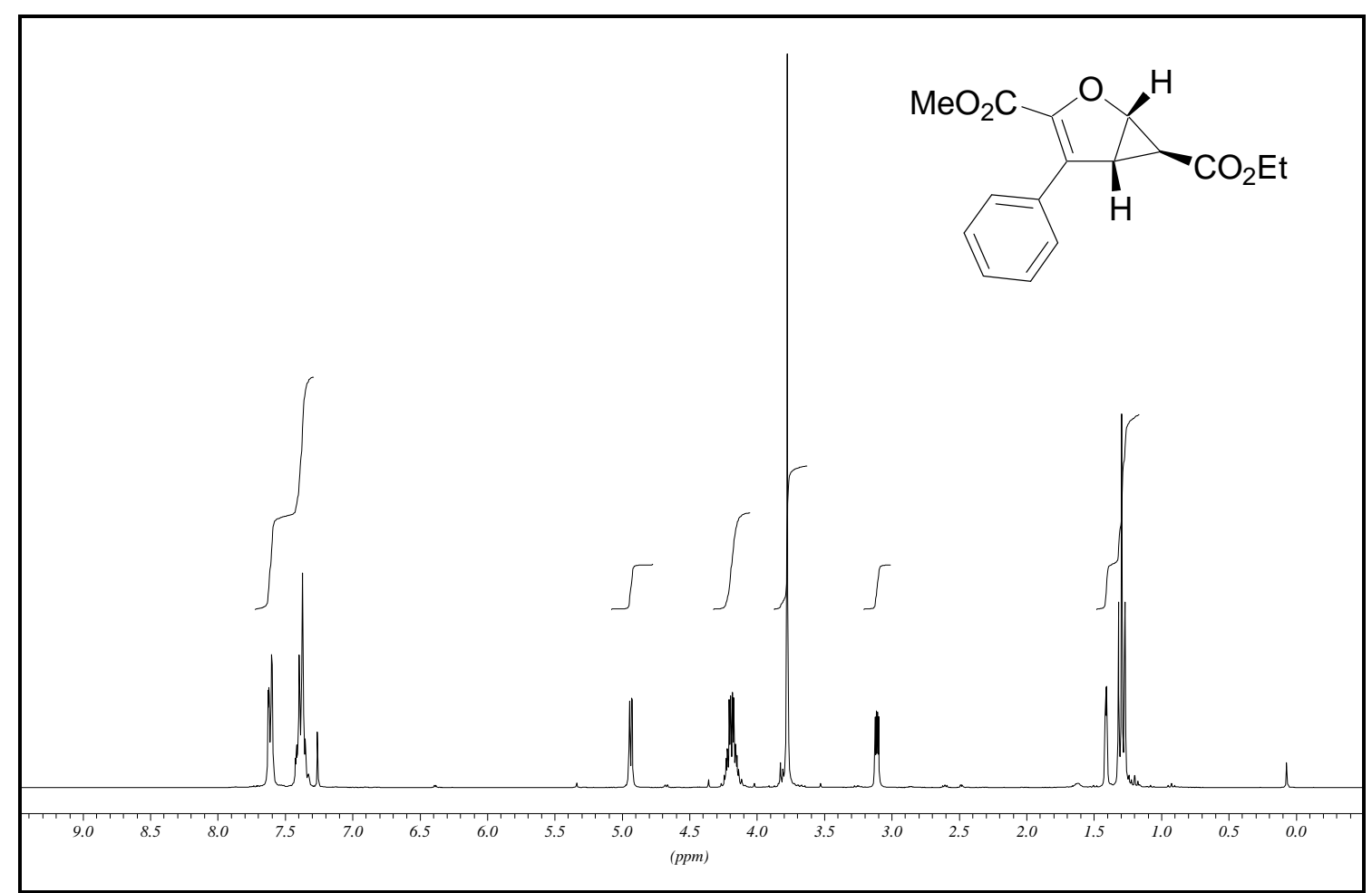

${ }^{13} \mathrm{C}-\mathrm{NMR}\left(75.5 \mathrm{MHz} \mathrm{CDCl}_{3}\right)$

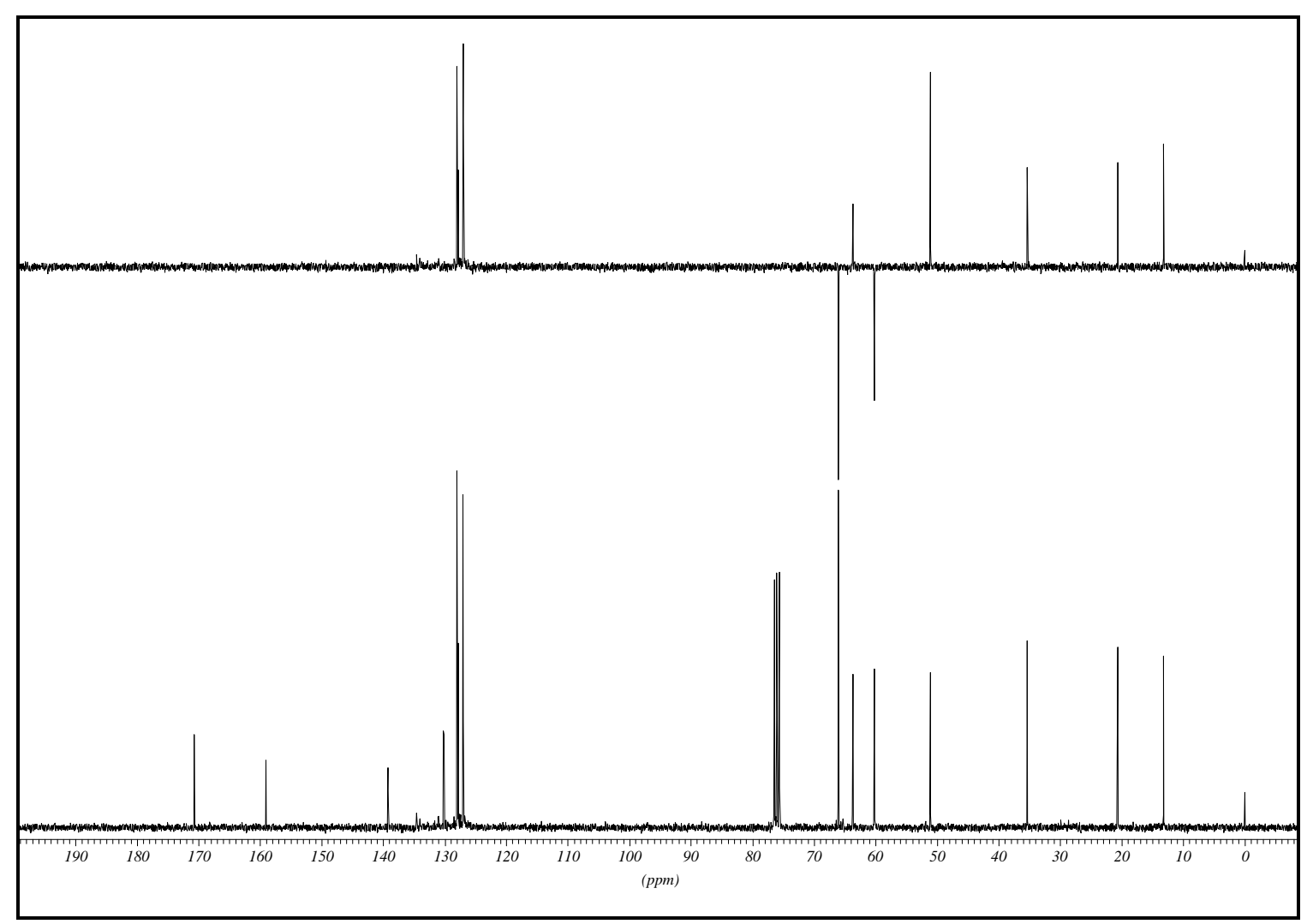


(1S,5R,6S)-(-)-4-(2-Methoxyphenyl)-2-oxa-bicyclo[3.1.0]hex-3-en-3,6-dicarbonsäure-6ethylester-3-methylester

${ }^{1}$ H-NMR (300 MHz, $\mathrm{CDCl}_{3}$ )

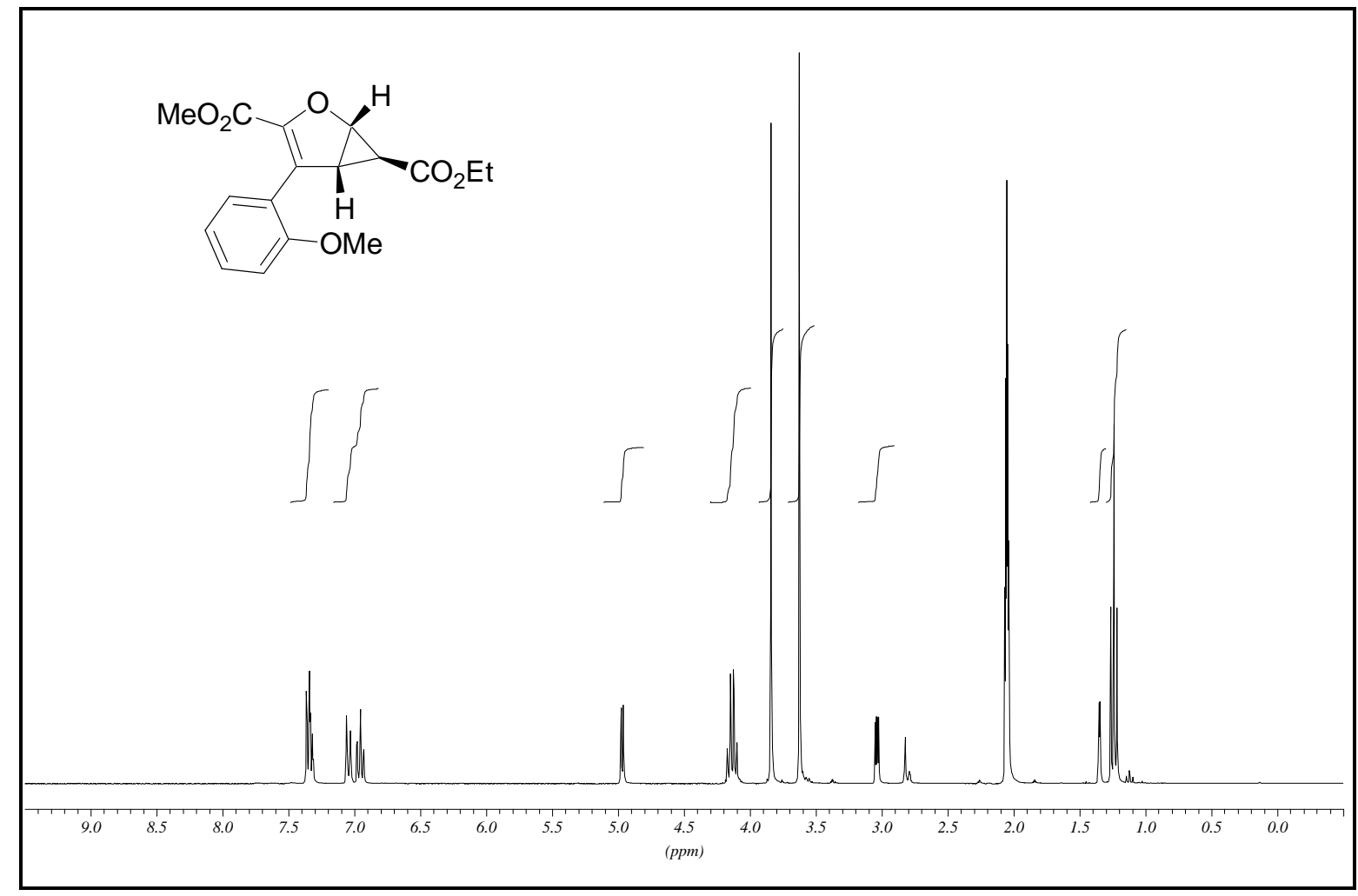

${ }^{13} \mathrm{C}-\mathrm{NMR}$ (75.5 MHz, $\mathrm{CDCl}_{3}$ )

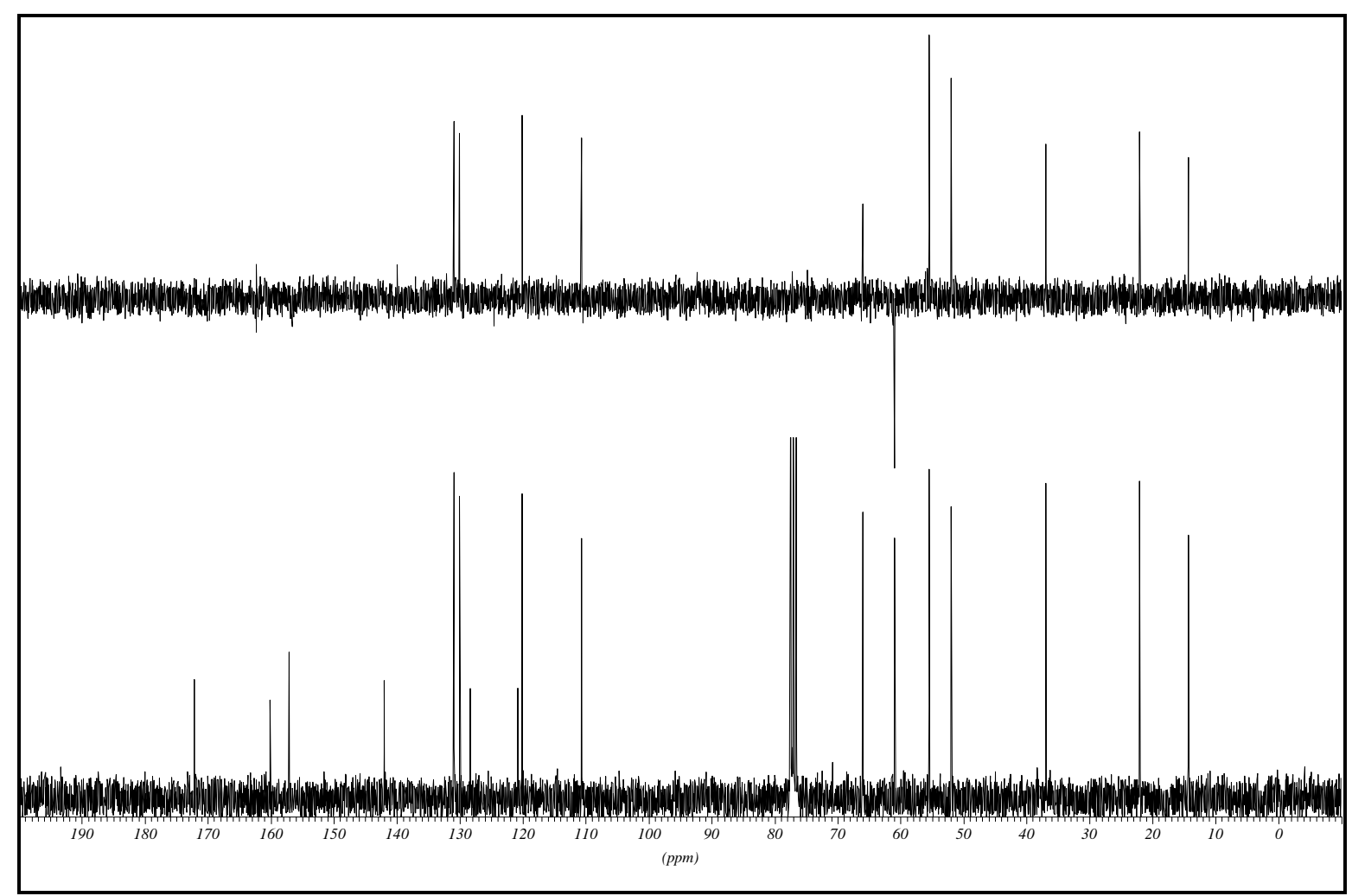


(1S,5R,6S)-(-)-4-Naphthalin-2-yl-2-oxa-bicyclo[3.1.0]hex-3-en-3,6-dicarbonsäure-6ethylester-3-methylester

${ }^{1} \mathrm{H}-\mathrm{NMR}$ (300 MHz, $\mathrm{CDCl}_{3}$ )

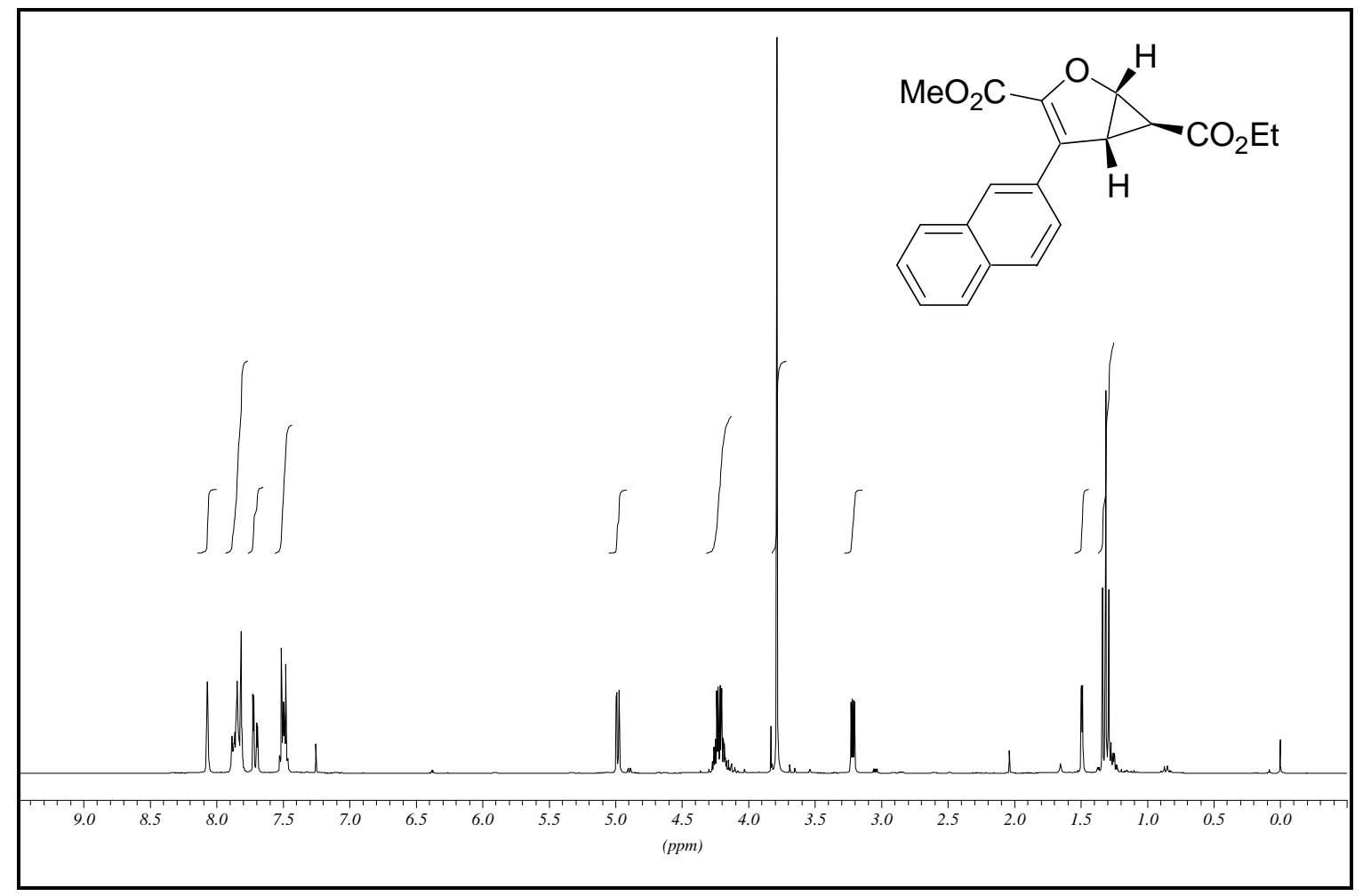

${ }^{13} \mathrm{C}-\mathrm{NMR}$ (75.5 MHz, $\mathrm{CDCl}_{3}$ )

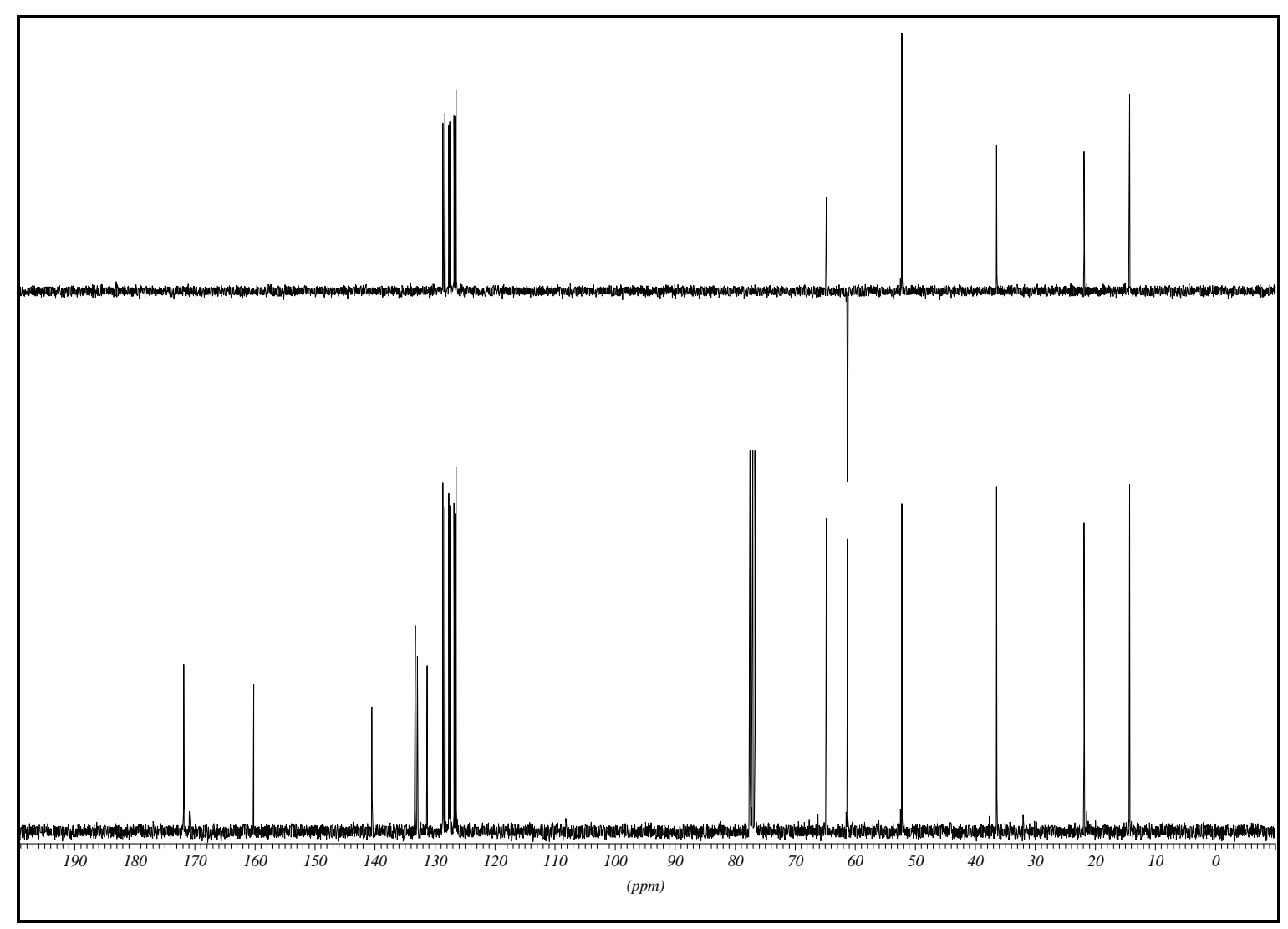


trans-(1S,5R,6S)-(-)-4-Styryl-2-oxa-bicyclo[3.1.0]hex-3-ene-3,6-dicarbonsäure-6ethylester-3-methylester

${ }^{1} \mathrm{H}-\mathrm{NMR}\left(300 \mathrm{MHz}, \mathrm{CDCl}_{3}\right)$

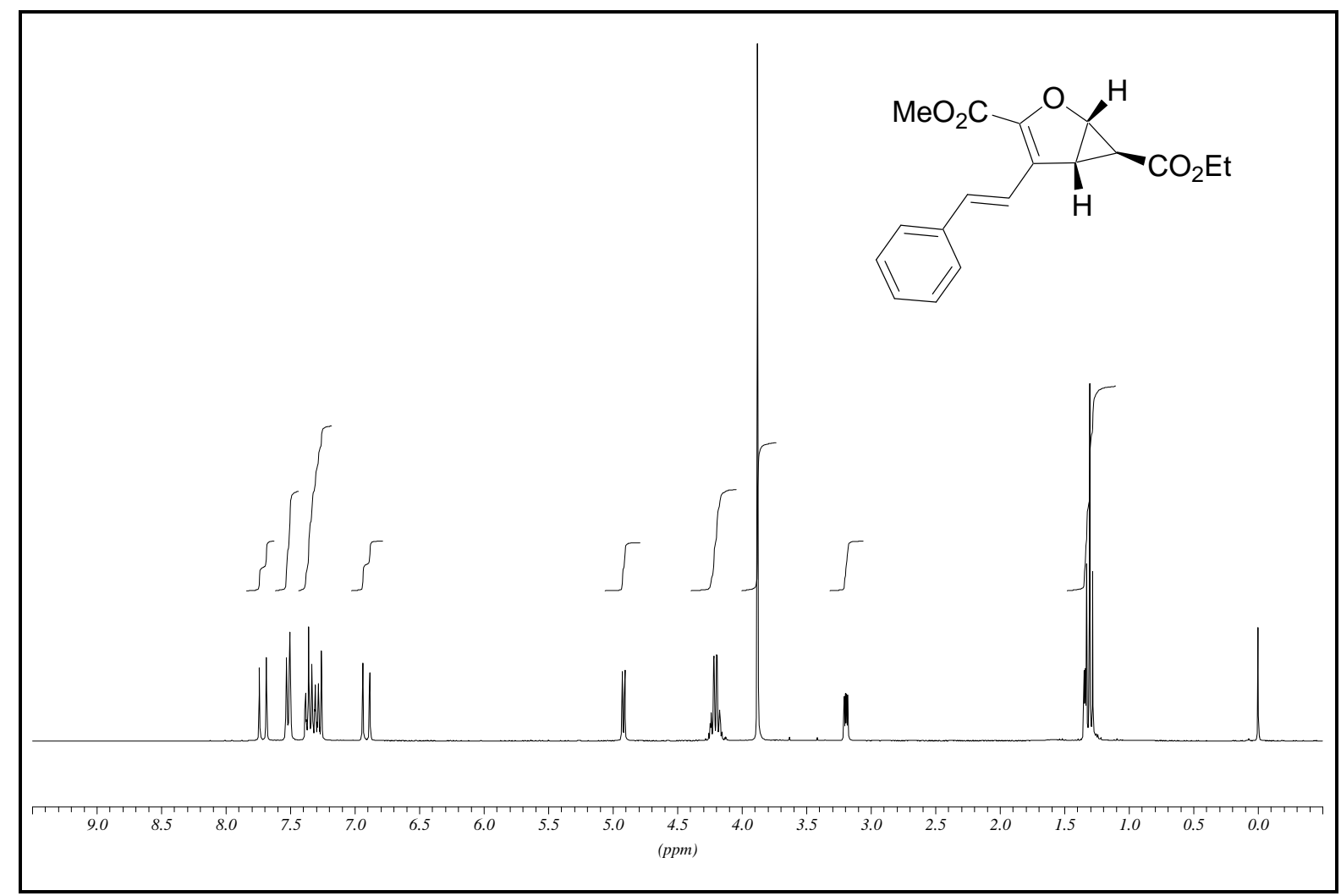

${ }^{13} \mathrm{C}-\mathrm{NMR}\left(75.5 \mathrm{MHz} \mathrm{CDCl}_{3}\right)$

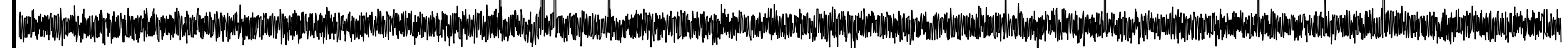

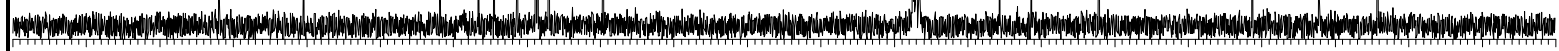
$\begin{array}{lllllllllllll}190 & 180 & 170 & 160 & 150 & 140 & 130 & 120 & 110 & 100 & 90 & 80\end{array}$ 
$(1 S, 3 R, 4 R, 5 S, 6 S)$-(-)-4-Phenyl-2-oxa-bicyclo[3.1.0]hexan-3,6-dicarbonsäure-6-ethylester3-methylester

${ }^{1} \mathrm{H}-\mathrm{NMR}$ (300 MHz, $\mathrm{CDCl}_{3}$ )

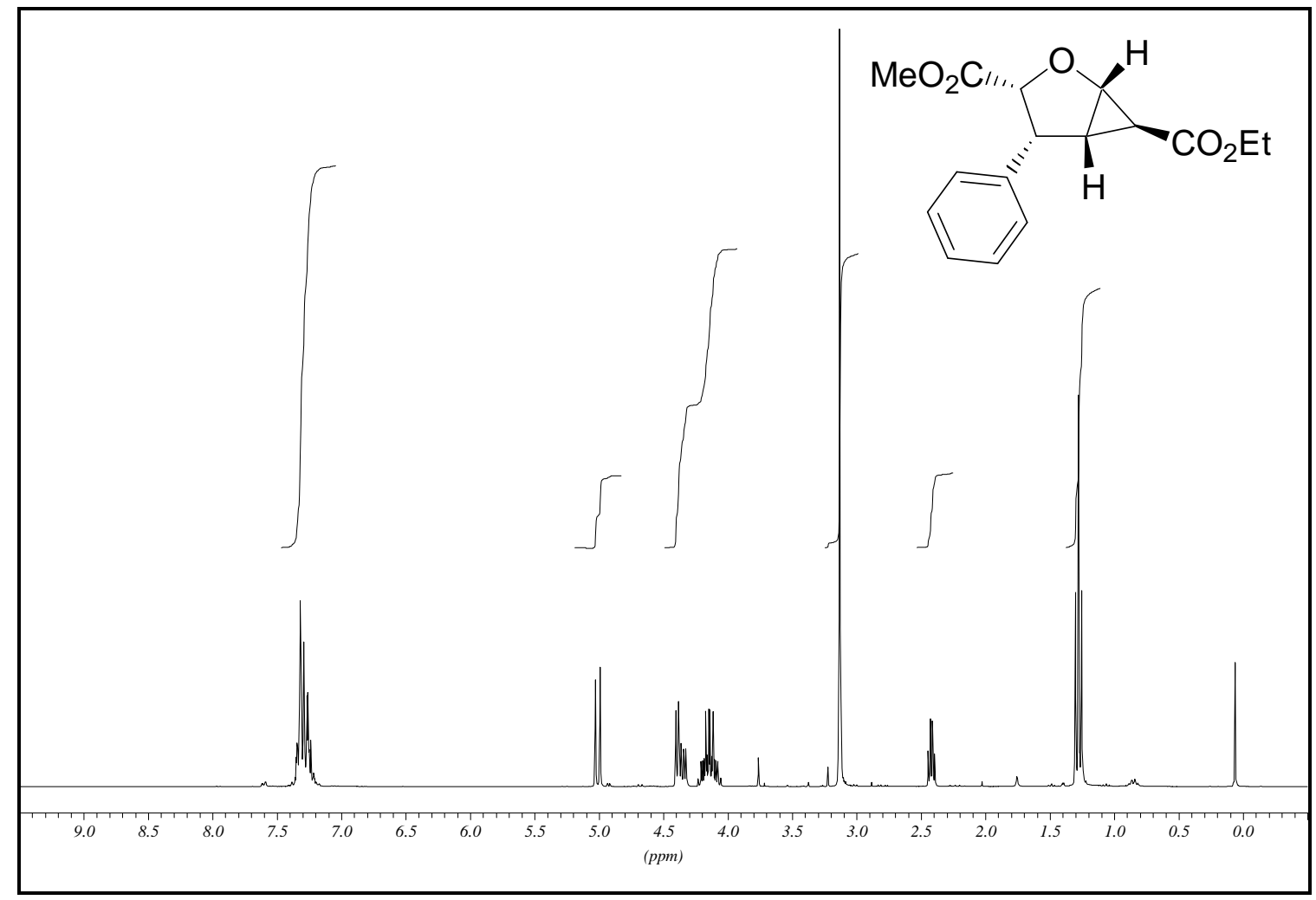

${ }^{13} \mathrm{C}$-NMR (75.5 MHz, $\mathrm{CDCl}_{3}$ )

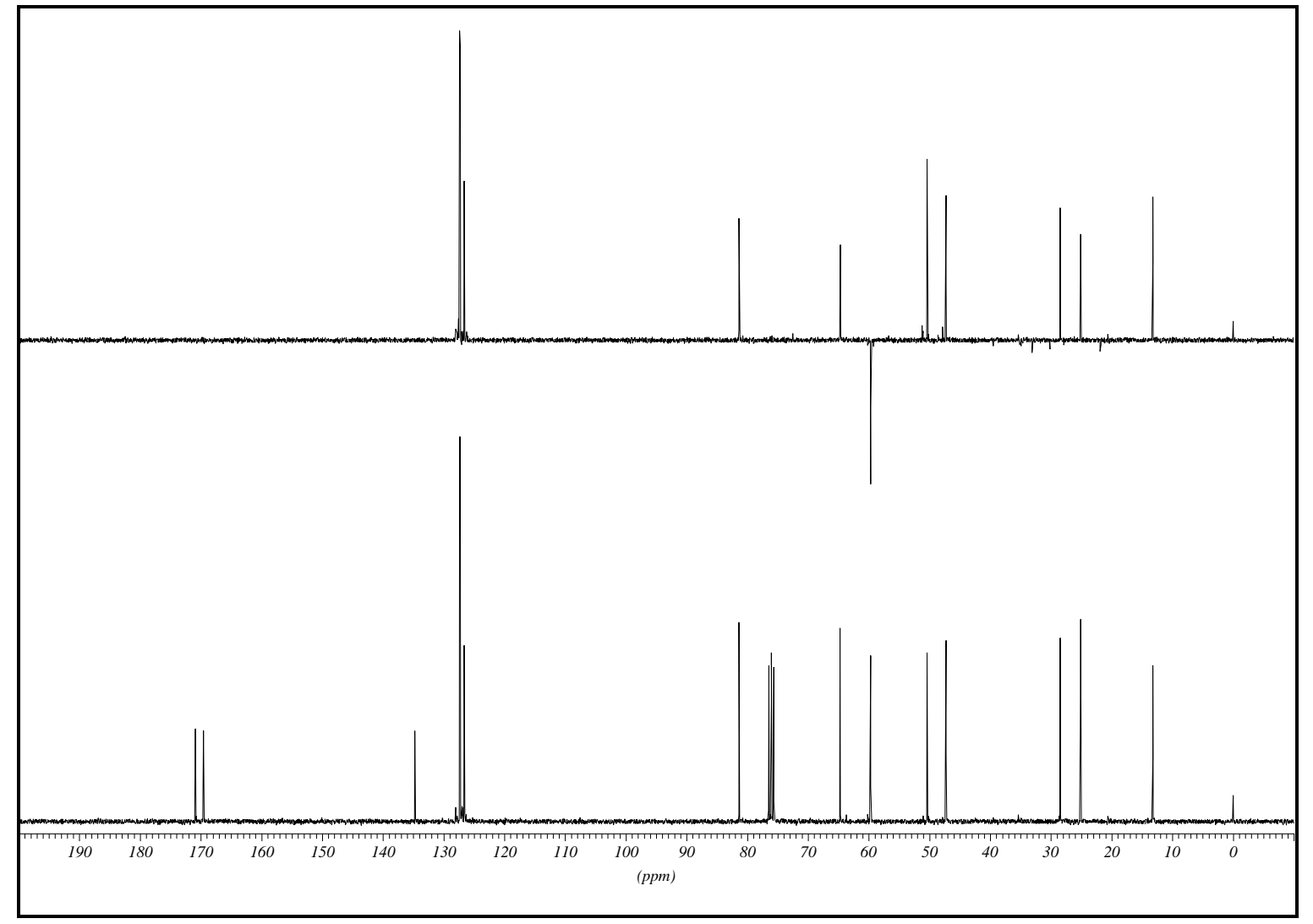


$(1 S, 3 R, 4 R, 5 S, 6 S)-(-)-4-N a p h t h a l i n-2-y l-2-0 x a-b i c y c l o[3.1 .0]$ hexan-3,6-dicarbonsäure-6ethylester-3-methylester

${ }^{1} \mathrm{H}$-NMR (300 MHz, $\mathrm{CDCl}_{3}$ )

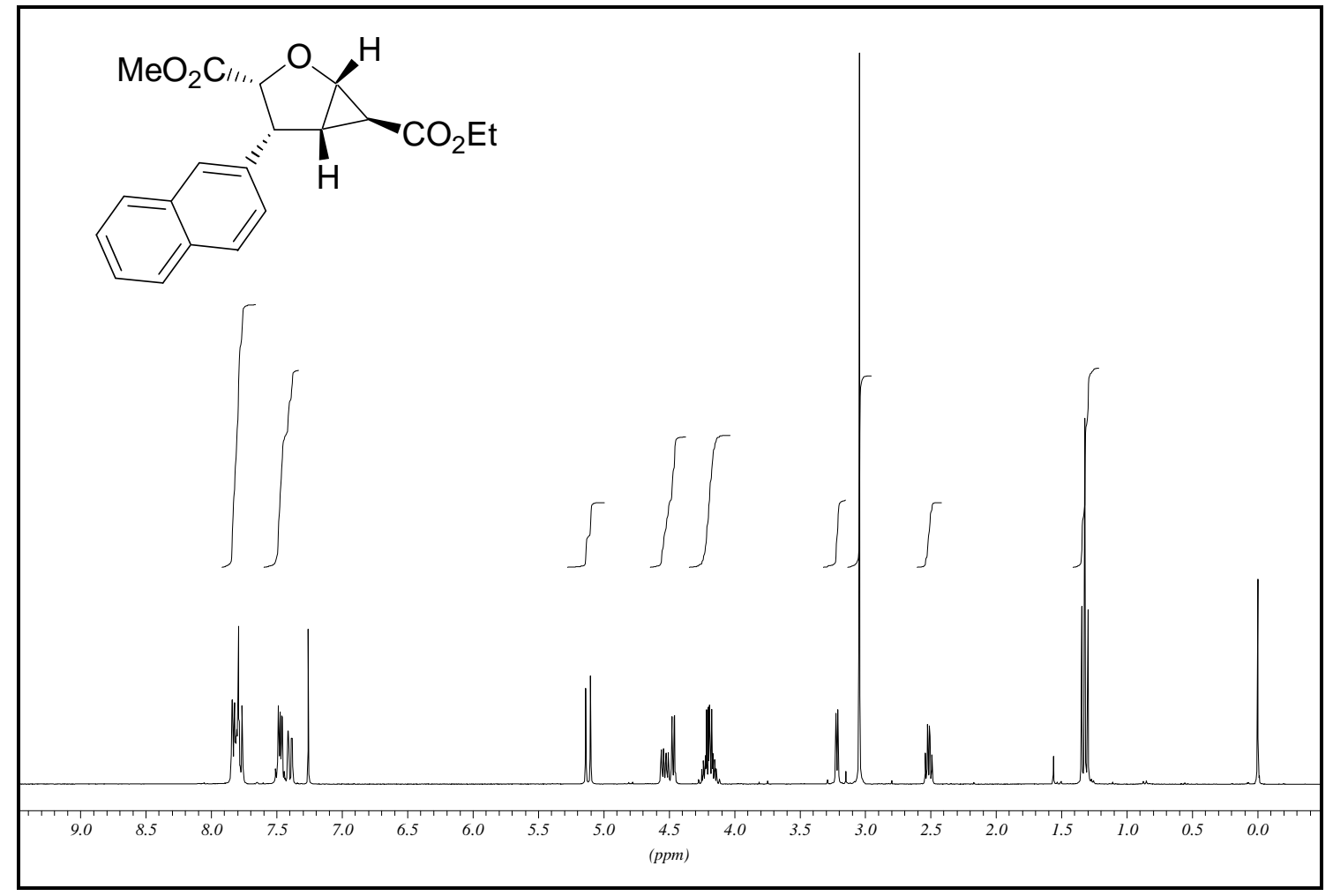

${ }^{13} \mathrm{C}-\mathrm{NMR}$ (75.5 MHz, $\mathrm{CDCl}_{3}$ )

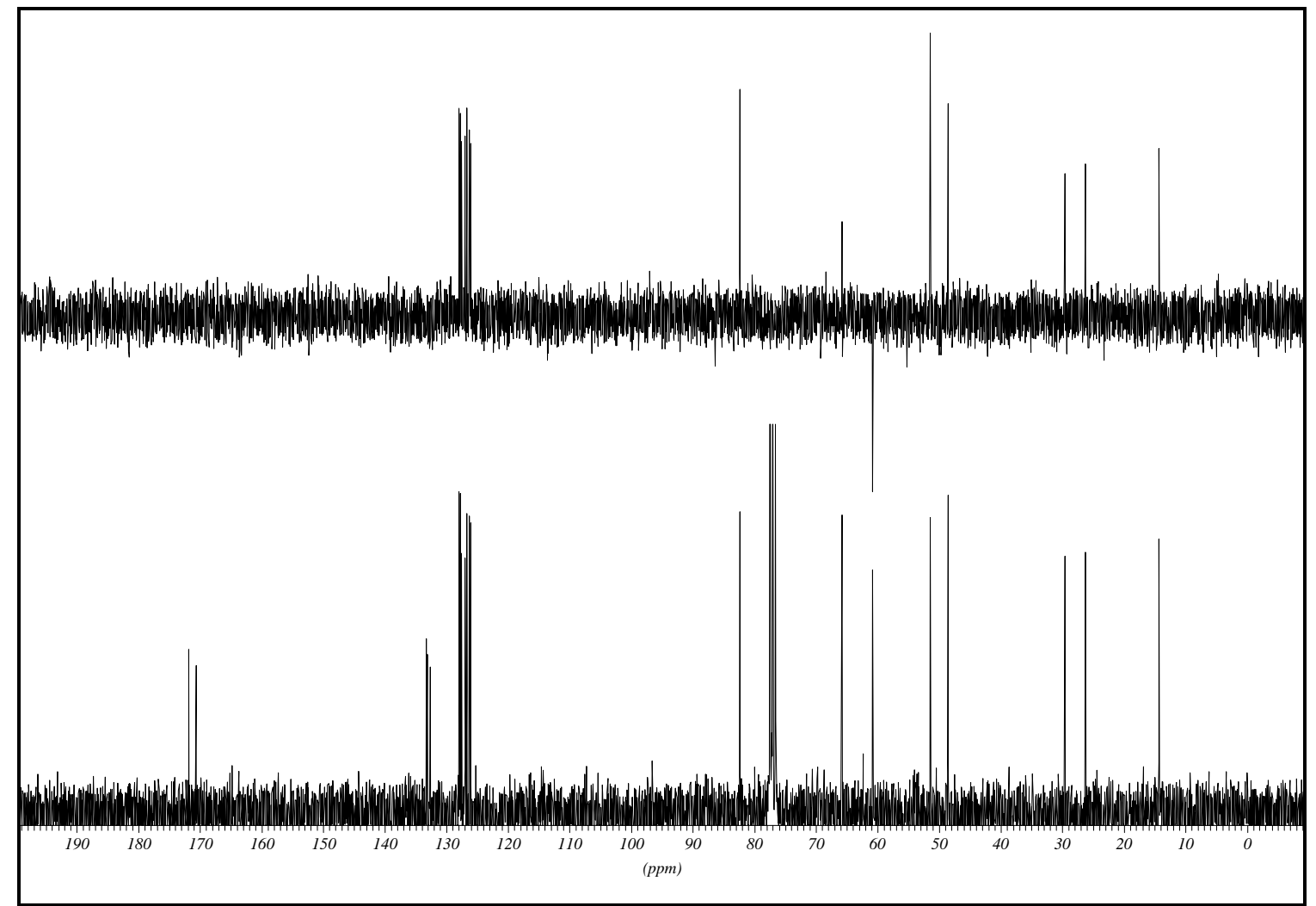


$(1 S, 3 R, 4 S, 5 S, 6 S)$-(+)-4-Phenethyl-2-oxa-bicyclo[3.1.0]hexan-3,6-dicarbonsäure-6ethylester-3-methylester

${ }^{1} \mathrm{H}-\mathrm{NMR}\left(600 \mathrm{MHz}, \mathrm{CDCl}_{3}\right)$

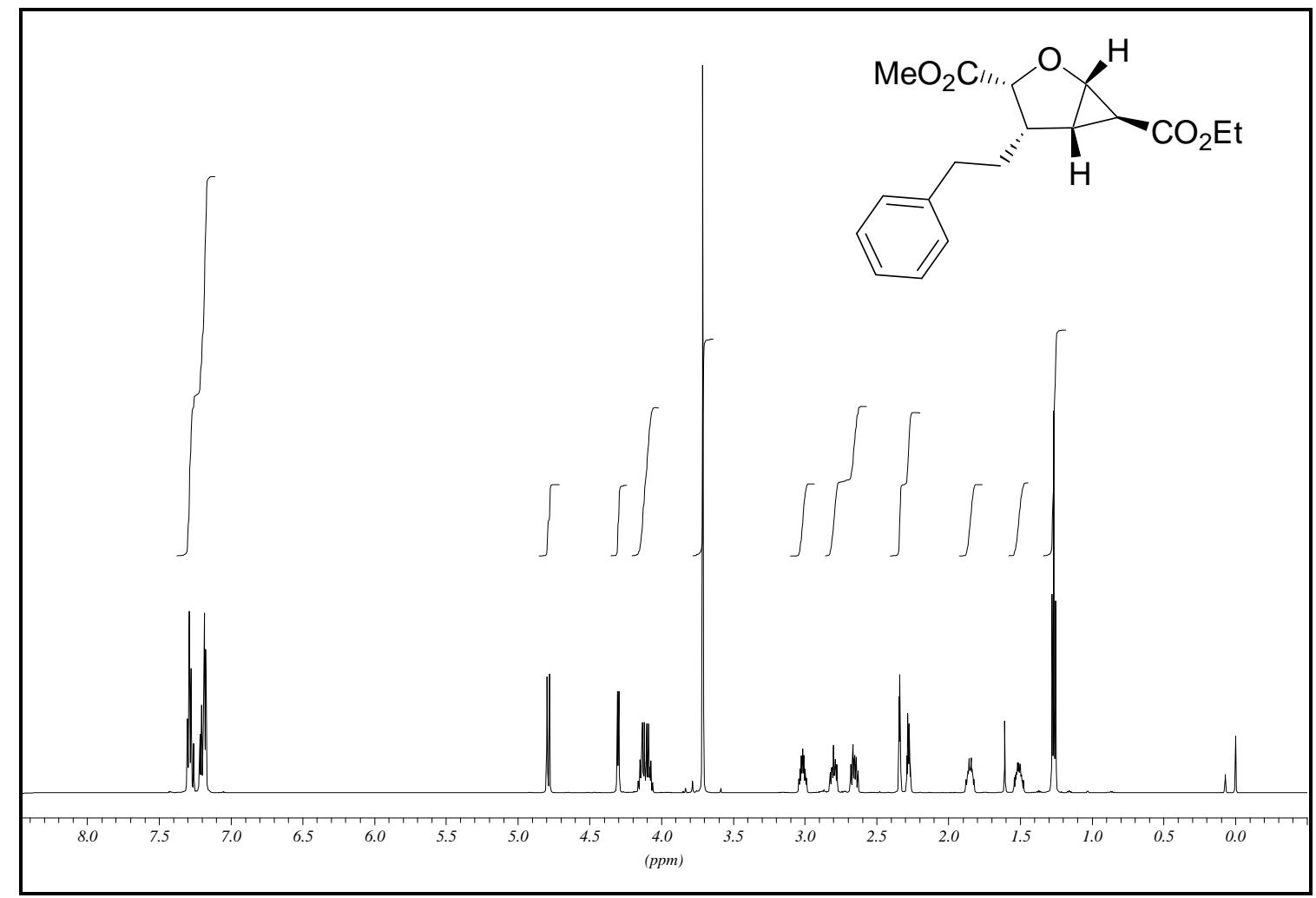

${ }^{13} \mathrm{C}$-NMR (75.5 MHz, $\left.\mathrm{CDCl}_{3}\right)$

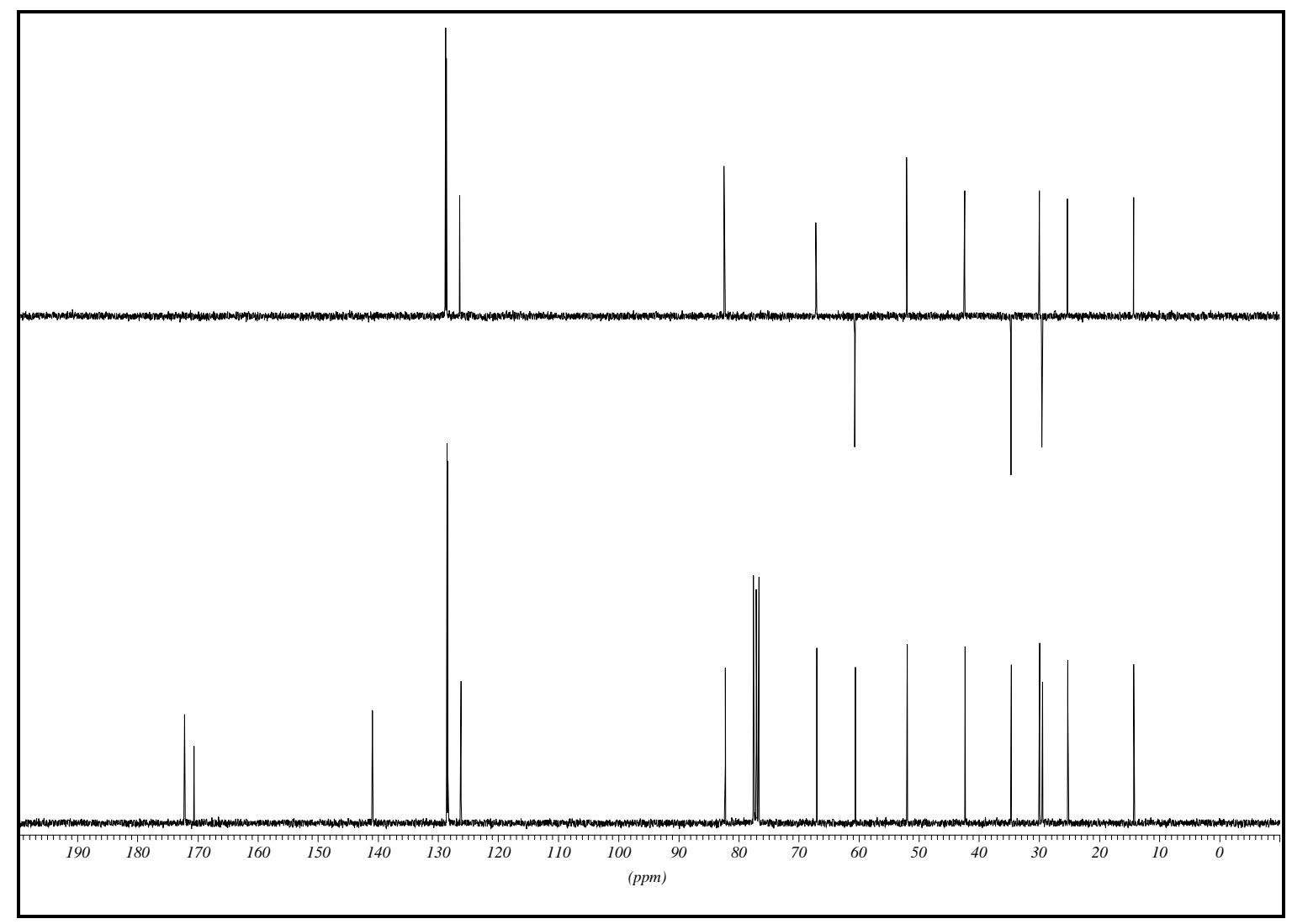


(2R,3R,3aS,6aS)-(-)-5-Oxo-3-phenyl-hexahydrofuro[2,3-b]furan-2-carbonsäure

${ }^{1}$ H-NMR (300 MHz, CD 3 OD)

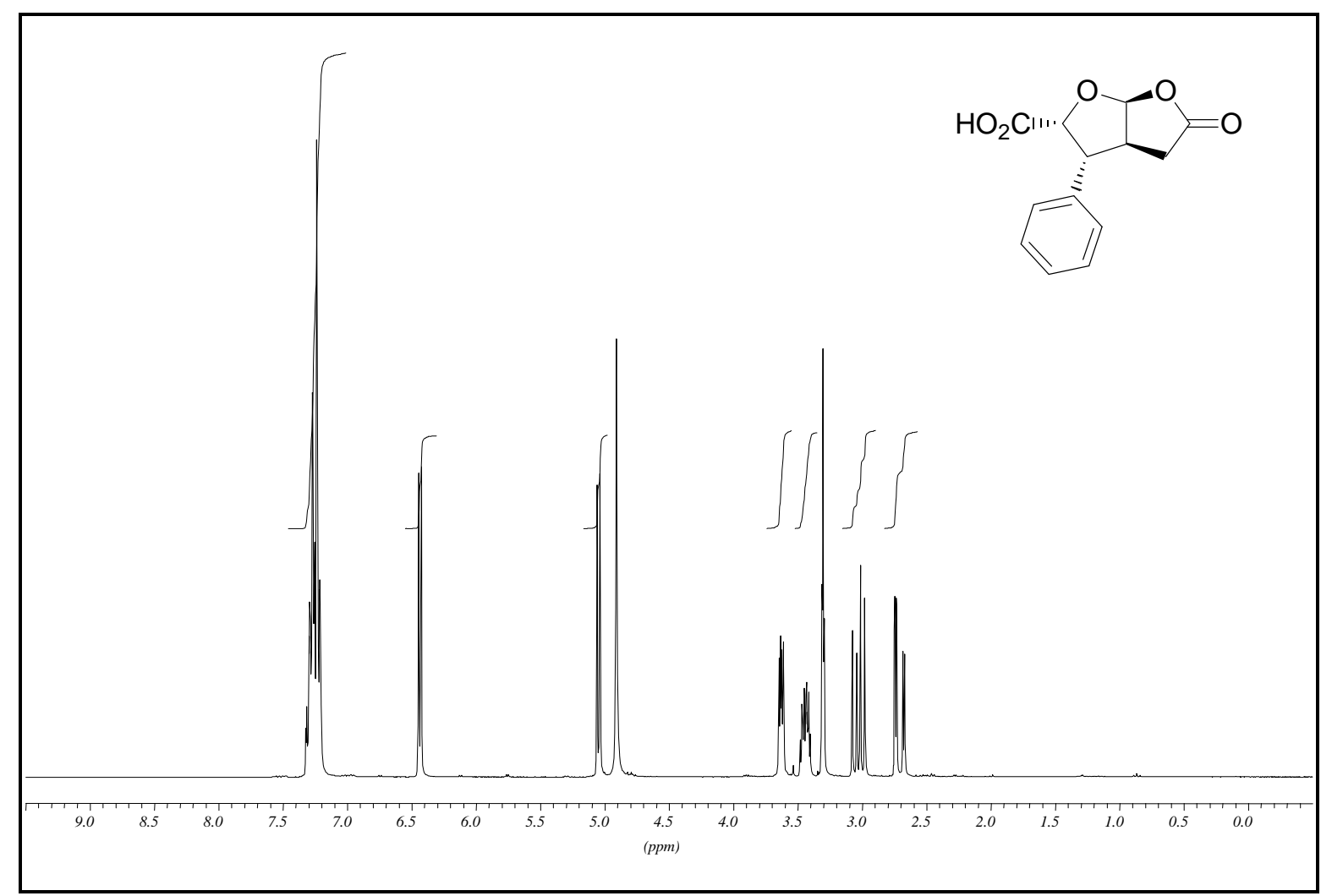

${ }^{13} \mathrm{C}-\mathrm{NMR}$ (75.5 MHz, CD $\mathrm{OD}$ )

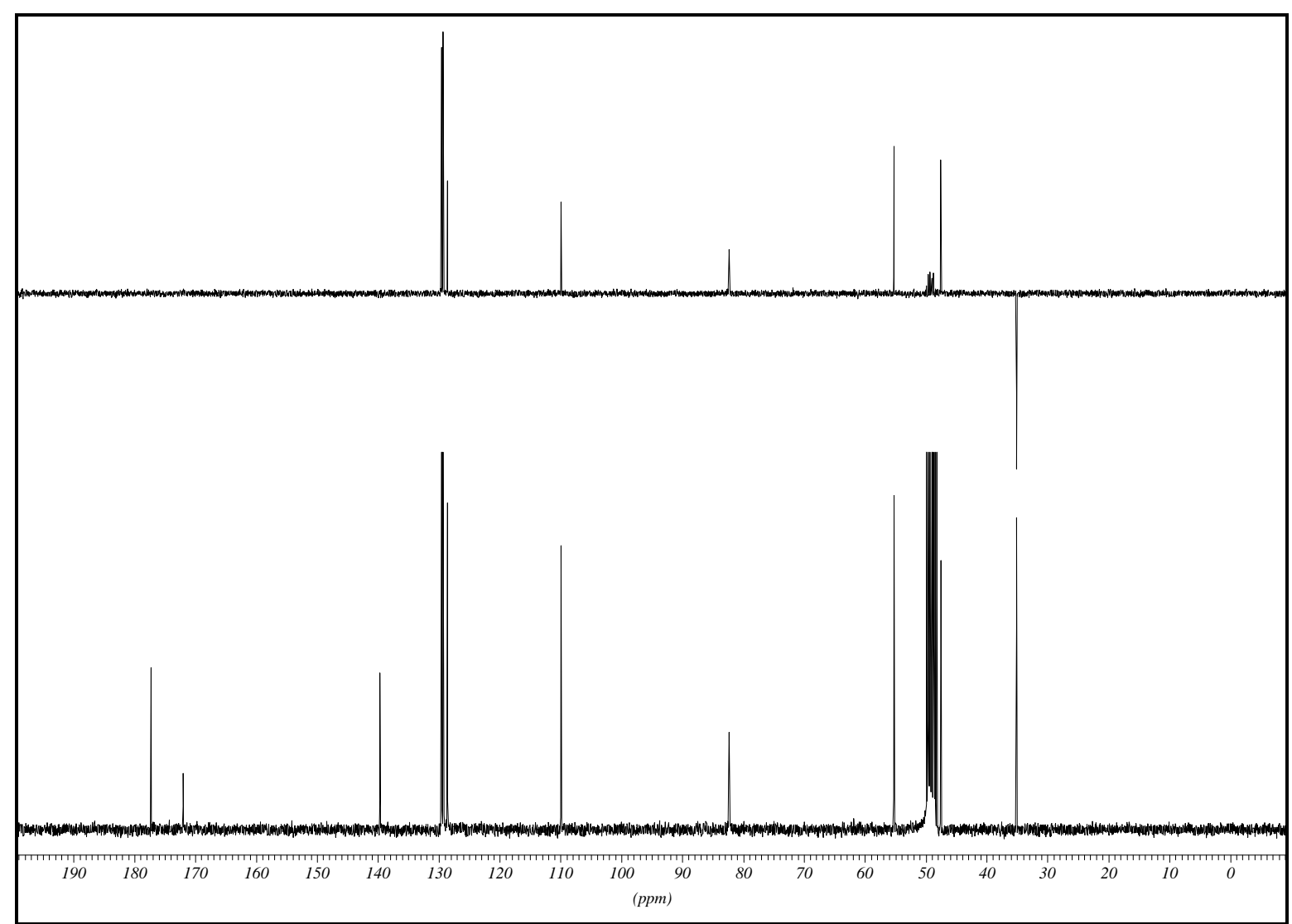


$(2 R, 3 R, 3 a S, 6 a S)-3-N a p h t h a l e n-2-y l-5-0 x 0-h e x a h y d r o f u r o[2,3-b]$ furan-2-carbonsäure ${ }^{1}$ H-NMR (400 MHz, CD 3 OD)

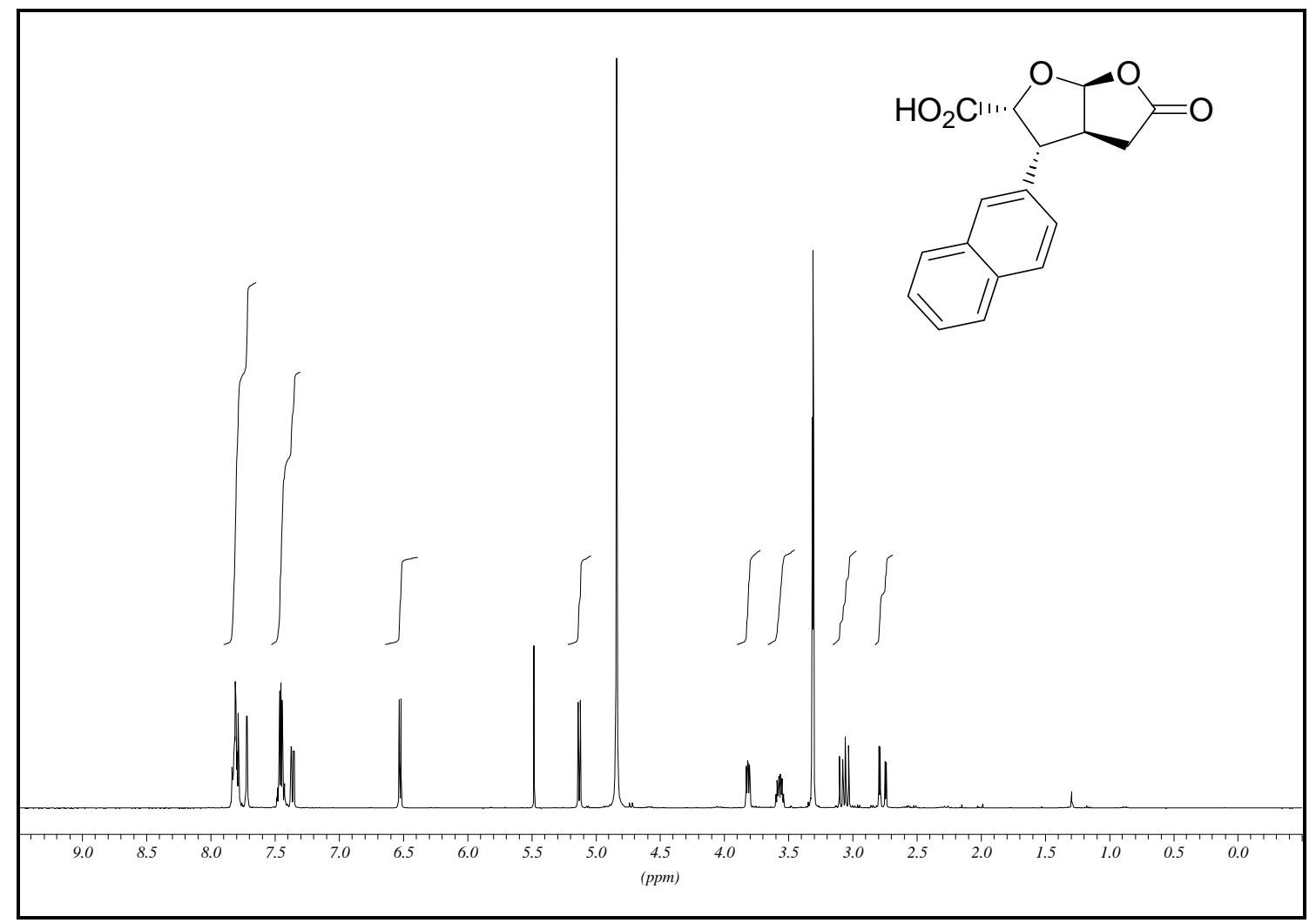

${ }^{13} \mathrm{C}-\mathrm{NMR}$ (75.5 MHz, CD $\mathrm{OD}$ )

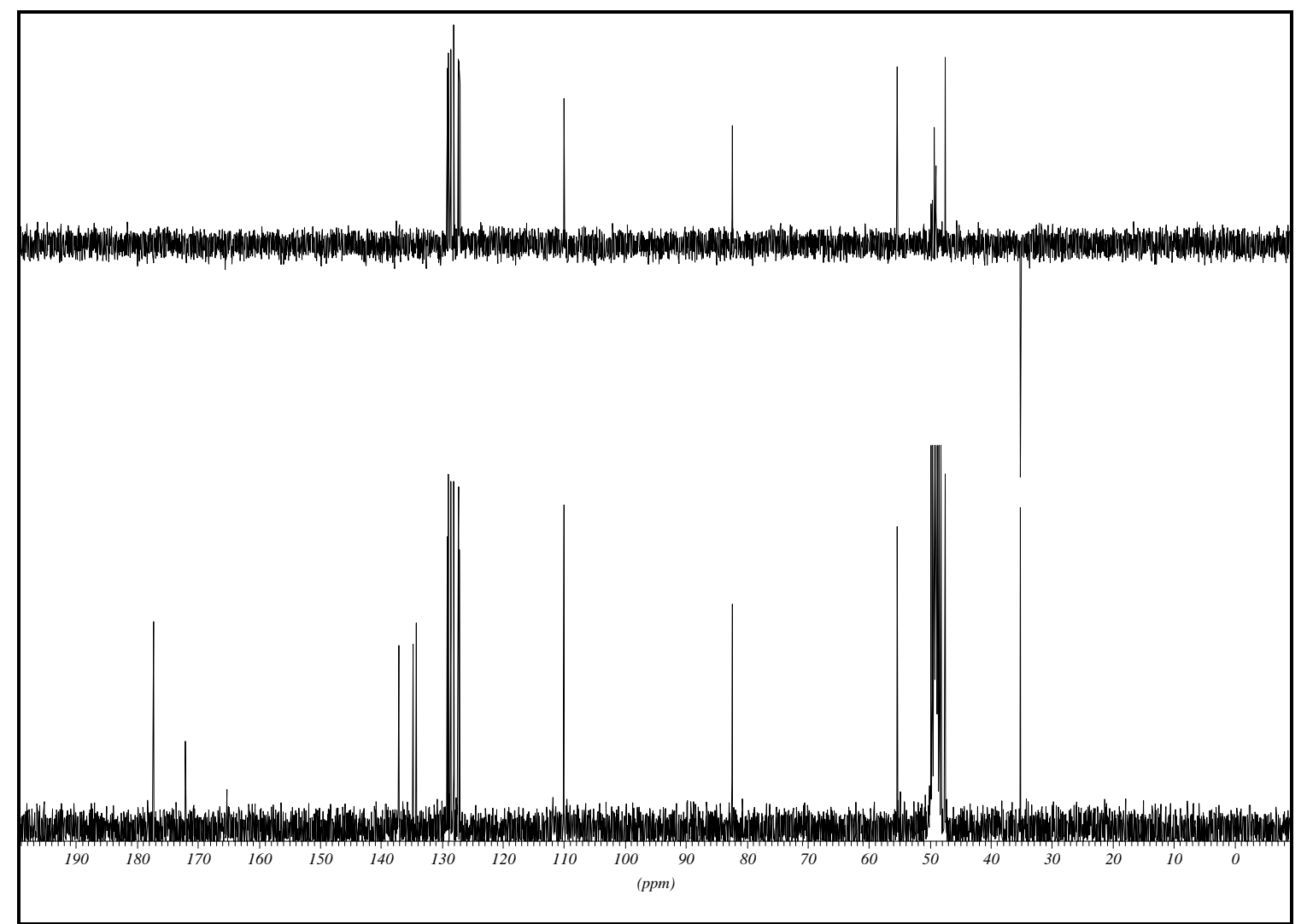


$(2 R, 3 S, 3 \mathrm{a} R, 6 \mathrm{a} R)-(-)-5-O x 0-3-p h e n e t h y l-h e x a h y d r o f u r o[2,3-b]$ furan-2-carbonsäure

${ }^{1}$ H-NMR (300 MHz, CD 3 OD)

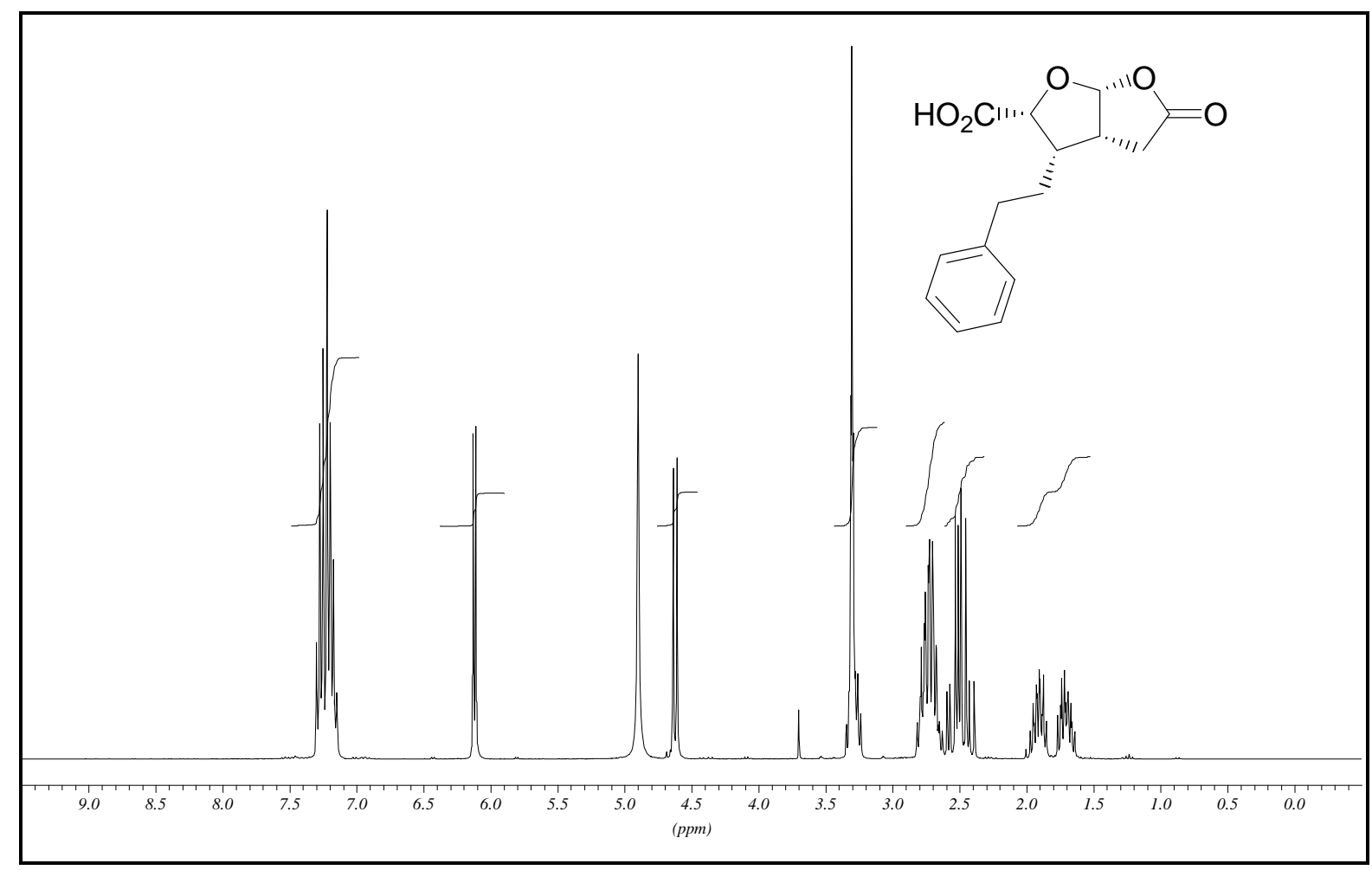

${ }^{13} \mathrm{C}-\mathrm{NMR}$ (75.5 MHz, CD $\left.3 \mathrm{OD}\right)$

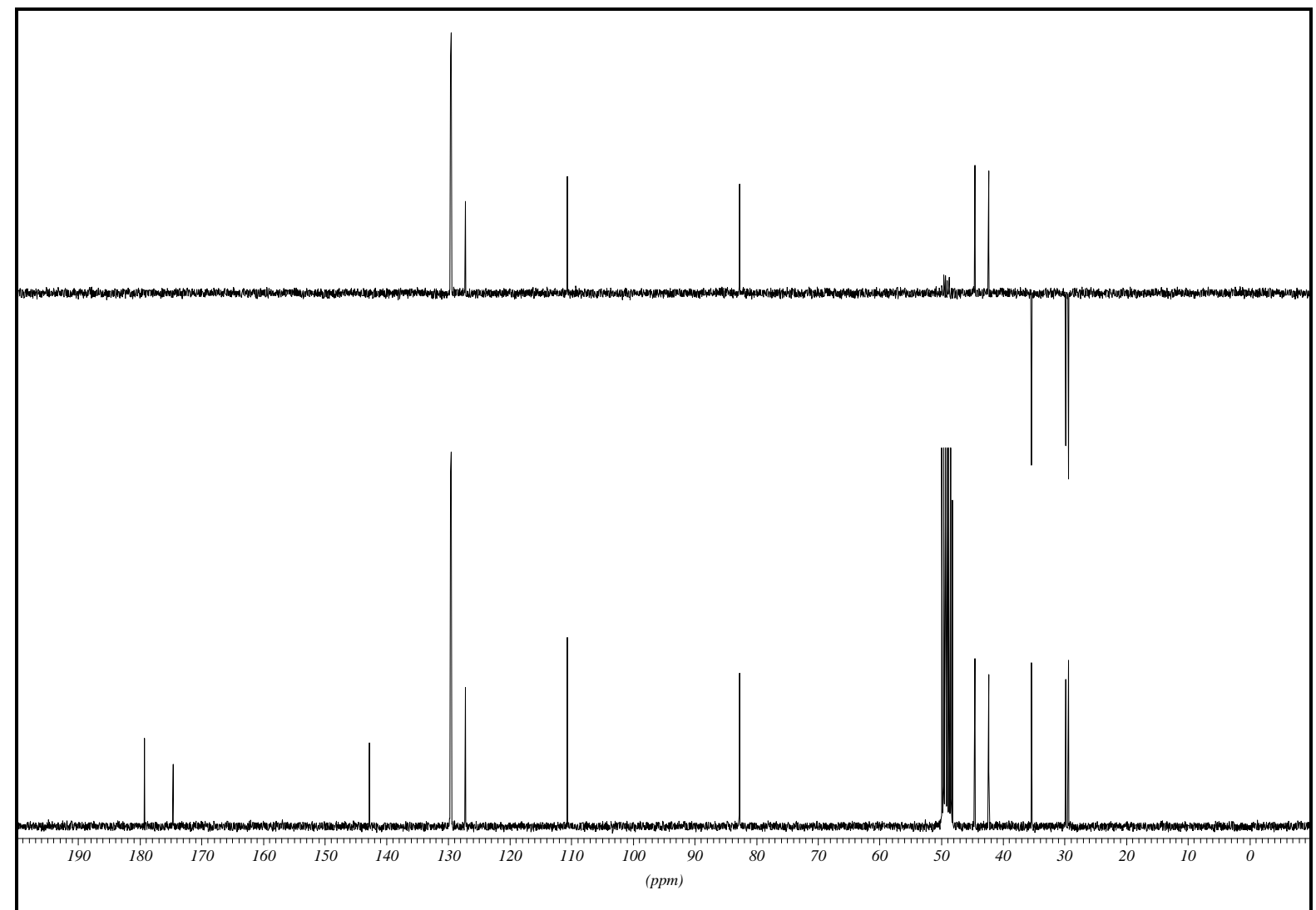


$(2 R, 3 R, 3 a S, 6 a S)-(-)-5-A c e t y l-4-p h e n y l-t e t r a h y d r o f u r o[2,3-b]$ furan-2-on

${ }^{1}$ H-NMR (300 MHz, $\mathrm{CDCl}_{3}$ )

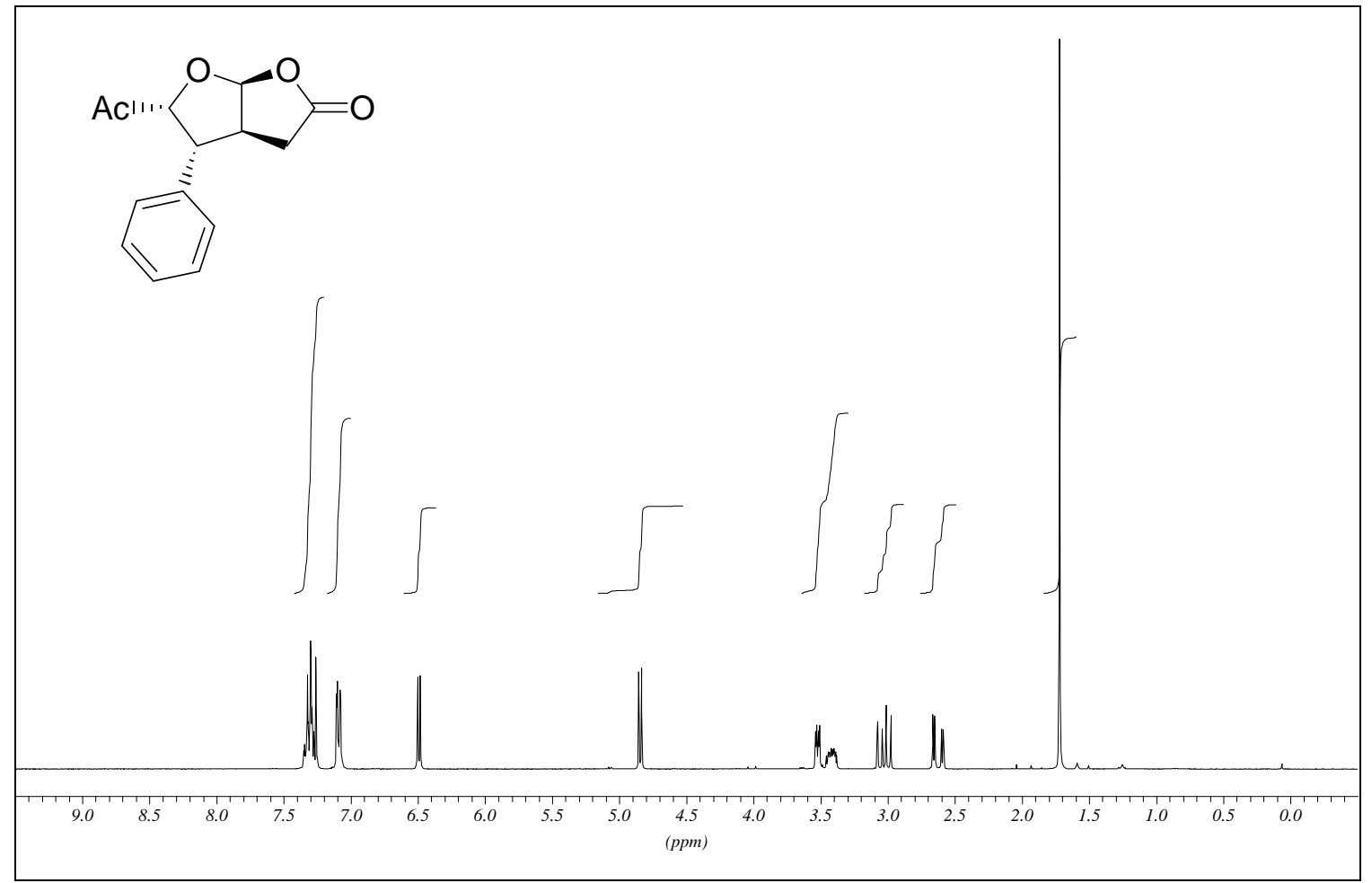

${ }^{13} \mathrm{C}$-NMR (75.5 MHz, $\mathrm{CDCl}_{3}$ )

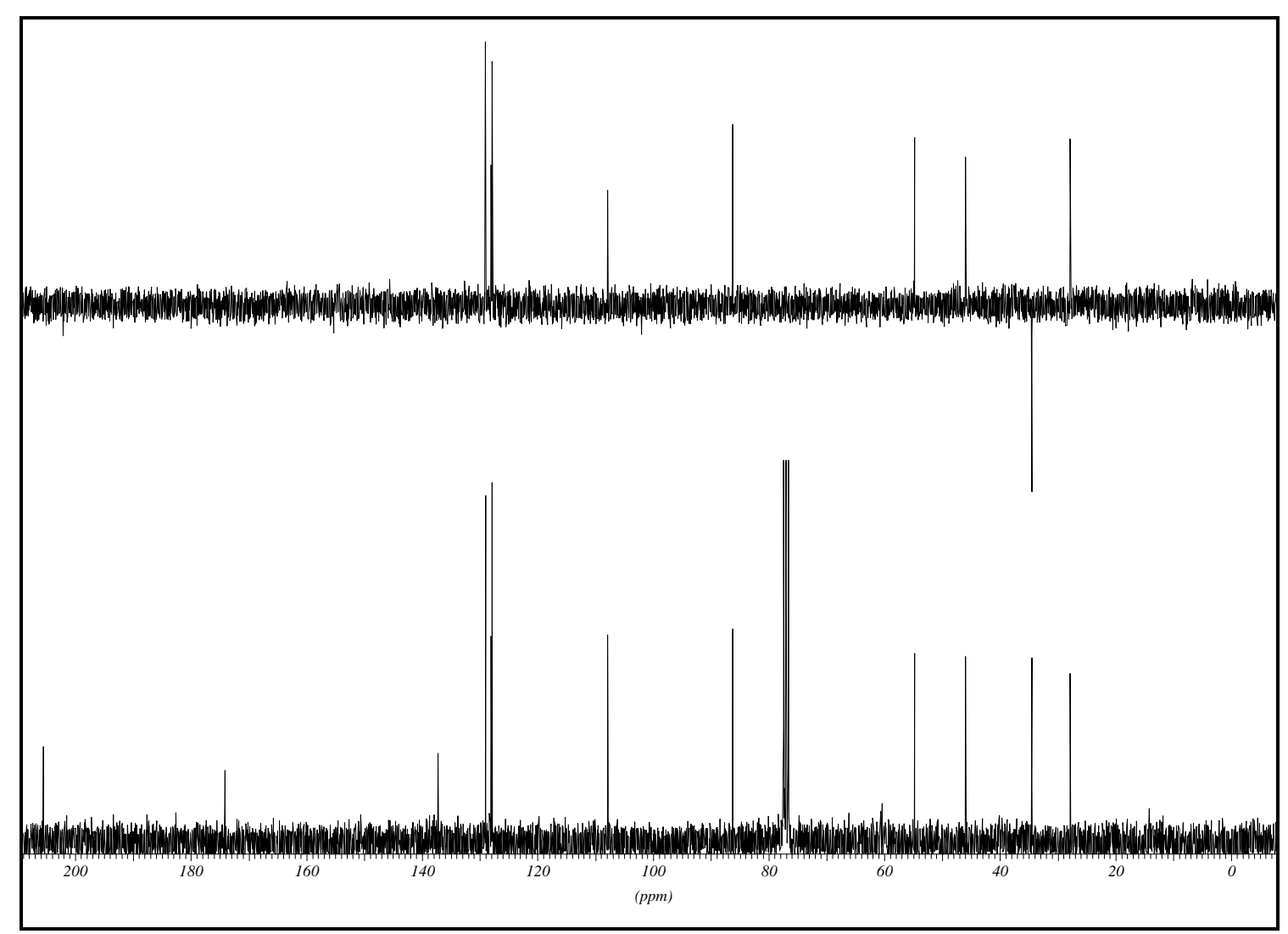


(2S,3R,3aS,6aS)-(-)-Essigsäure-5-oxo-3-phenyl-hexahydrofuro[2,3-b]furan-2-ylester ${ }^{1} \mathrm{H}-\mathrm{NMR}\left(300 \mathrm{MHz}, \mathrm{CDCl}_{3}\right)$

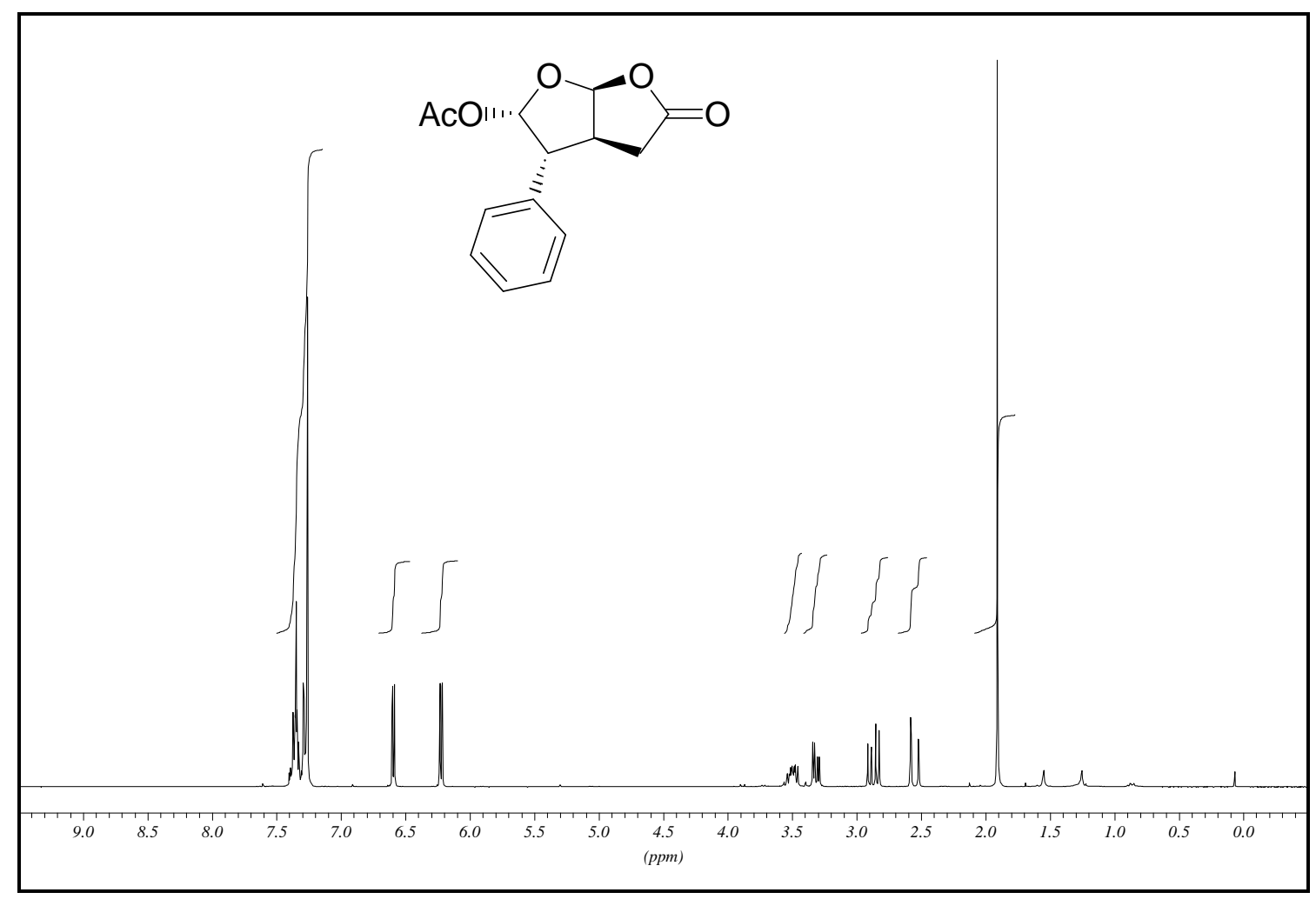

${ }^{13} \mathrm{C}-\mathrm{NMR}\left(75.5 \mathrm{MHz} \mathrm{CDCl}_{3}\right)$

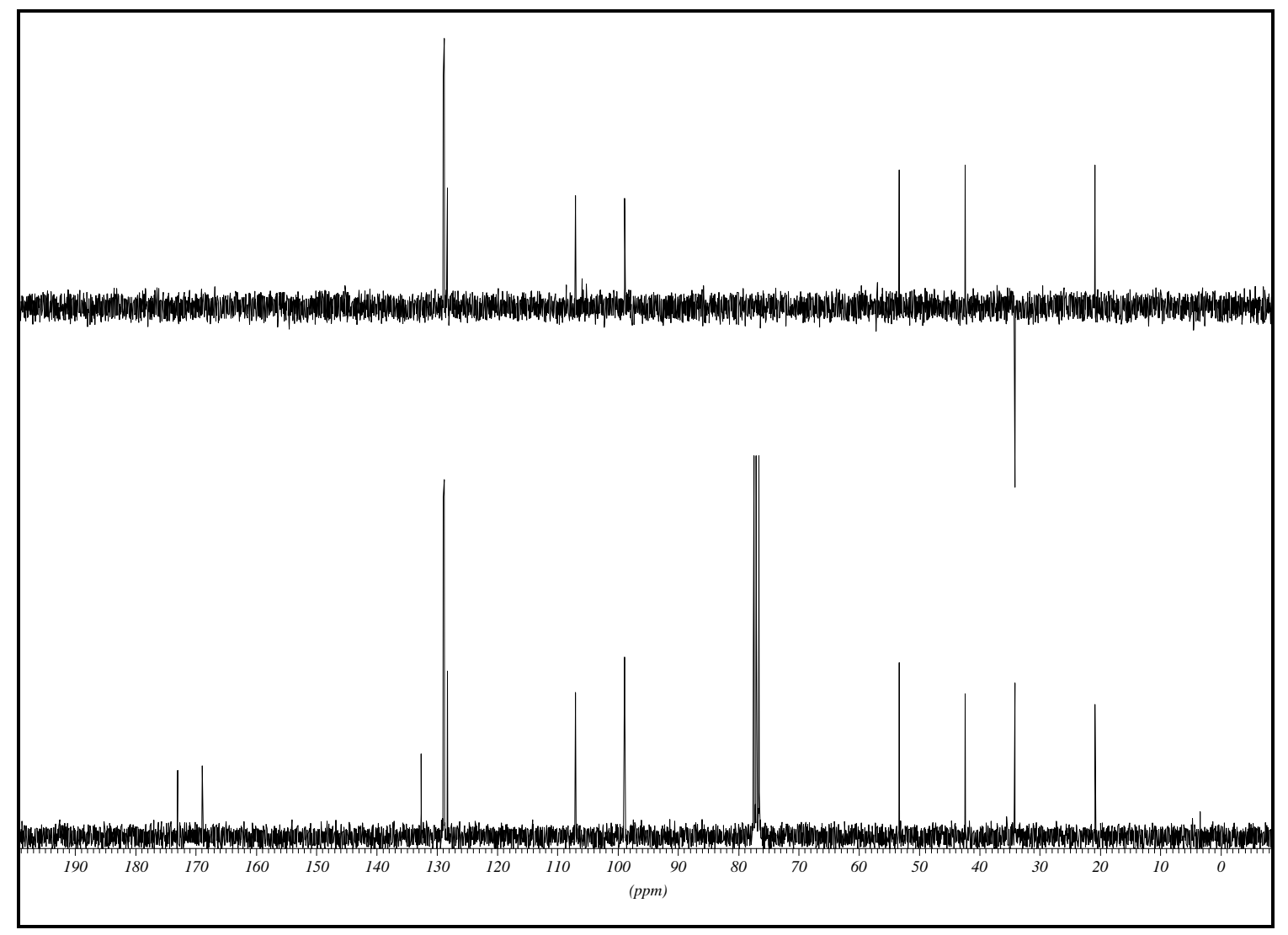




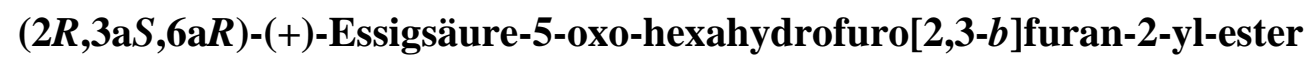

${ }^{1} \mathrm{H}-\mathrm{NMR}\left(300 \mathrm{MHz}, \mathrm{CDCl}_{3}\right)$

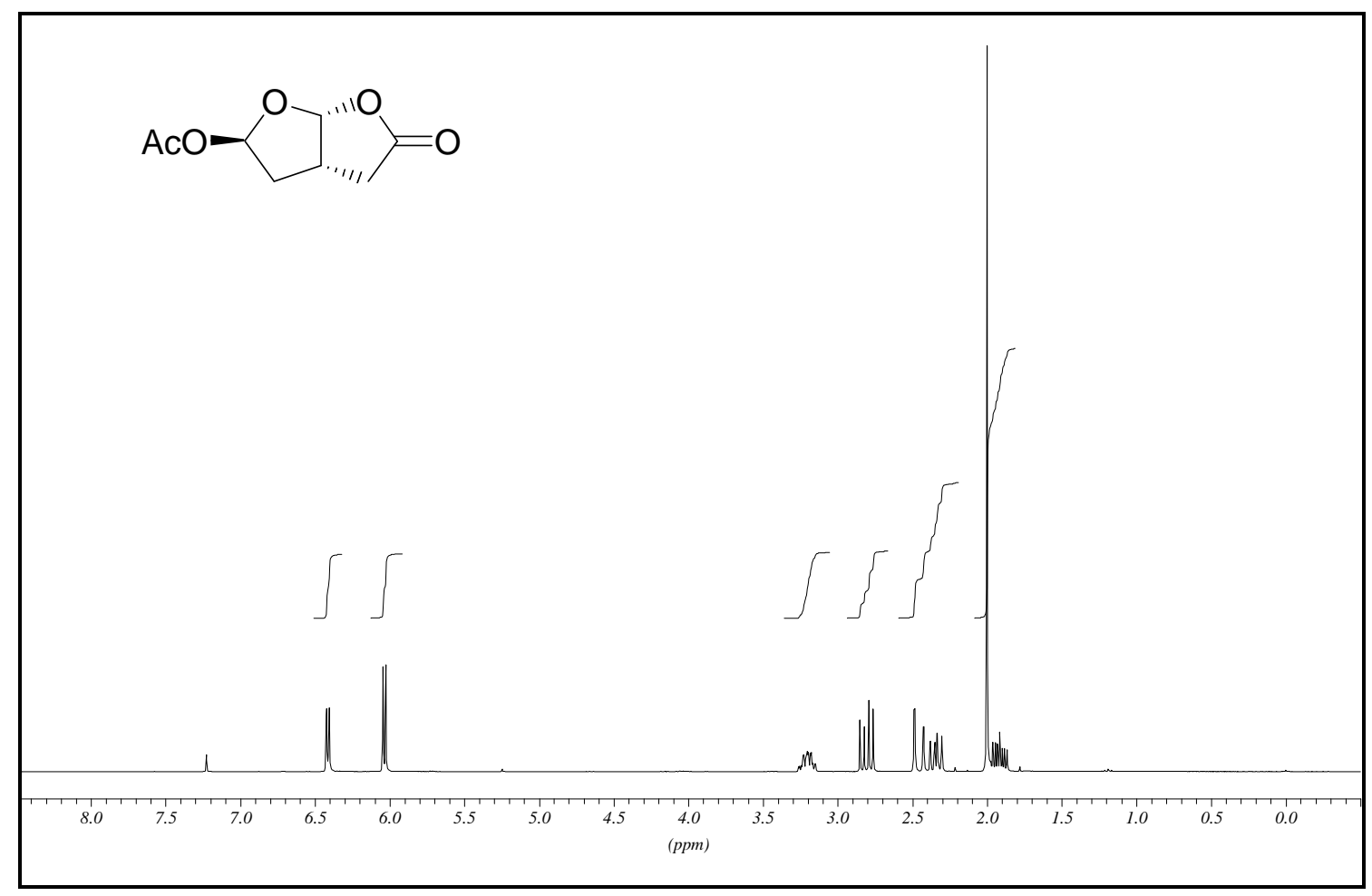

${ }^{13} \mathrm{C}-\mathrm{NMR}\left(75 \mathrm{MHz}, \mathrm{CDCl}_{3}\right)$

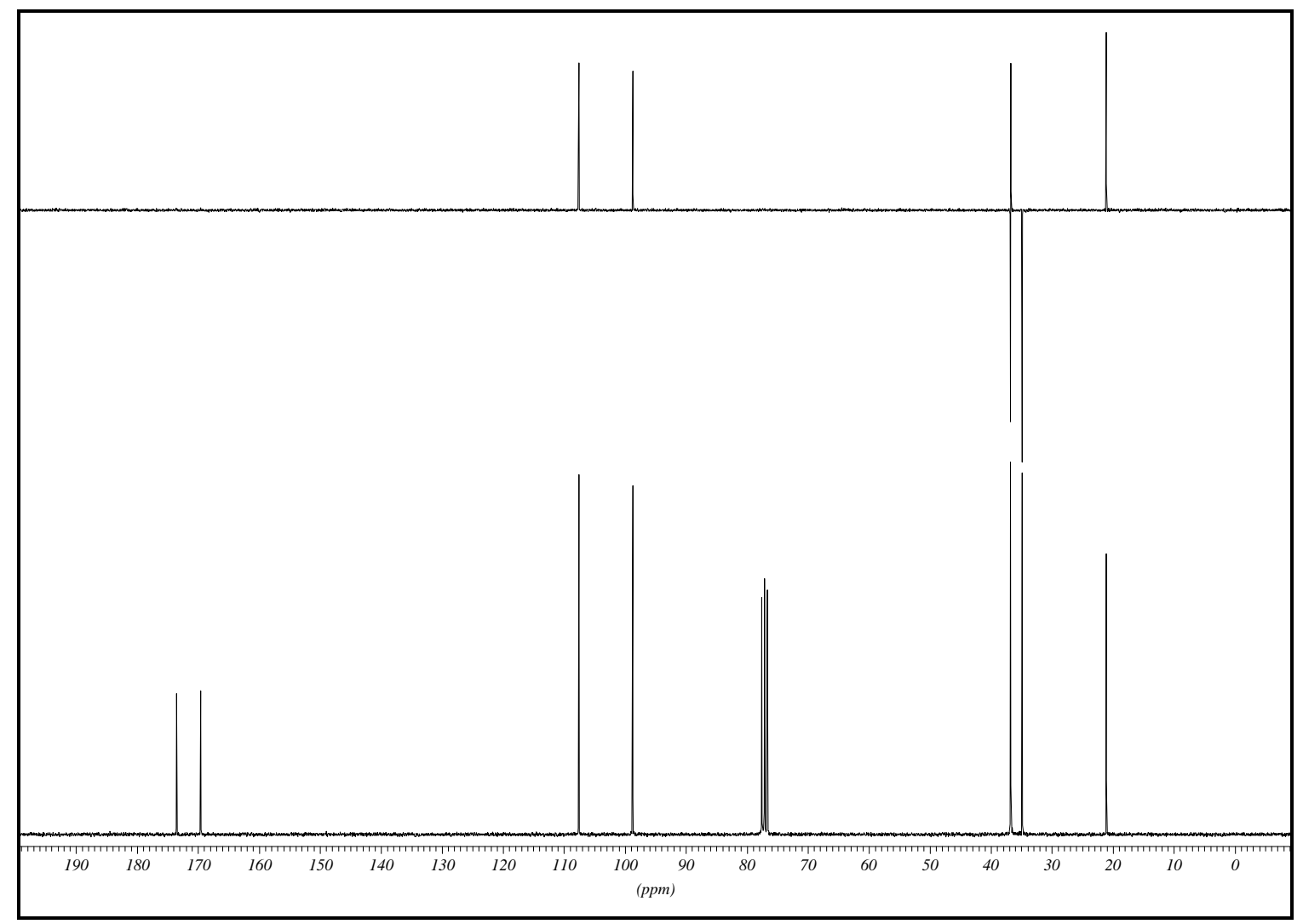


(2R/S,3S,3a $R, 6 \mathrm{a} R)$-Essigsäure-5-oxo-3-phenethyl-hexahydrofuro[2,3-b]furan-2-yl-ester

${ }^{1}$ H-NMR (300 MHz, $\mathrm{CDCl}_{3}$ )

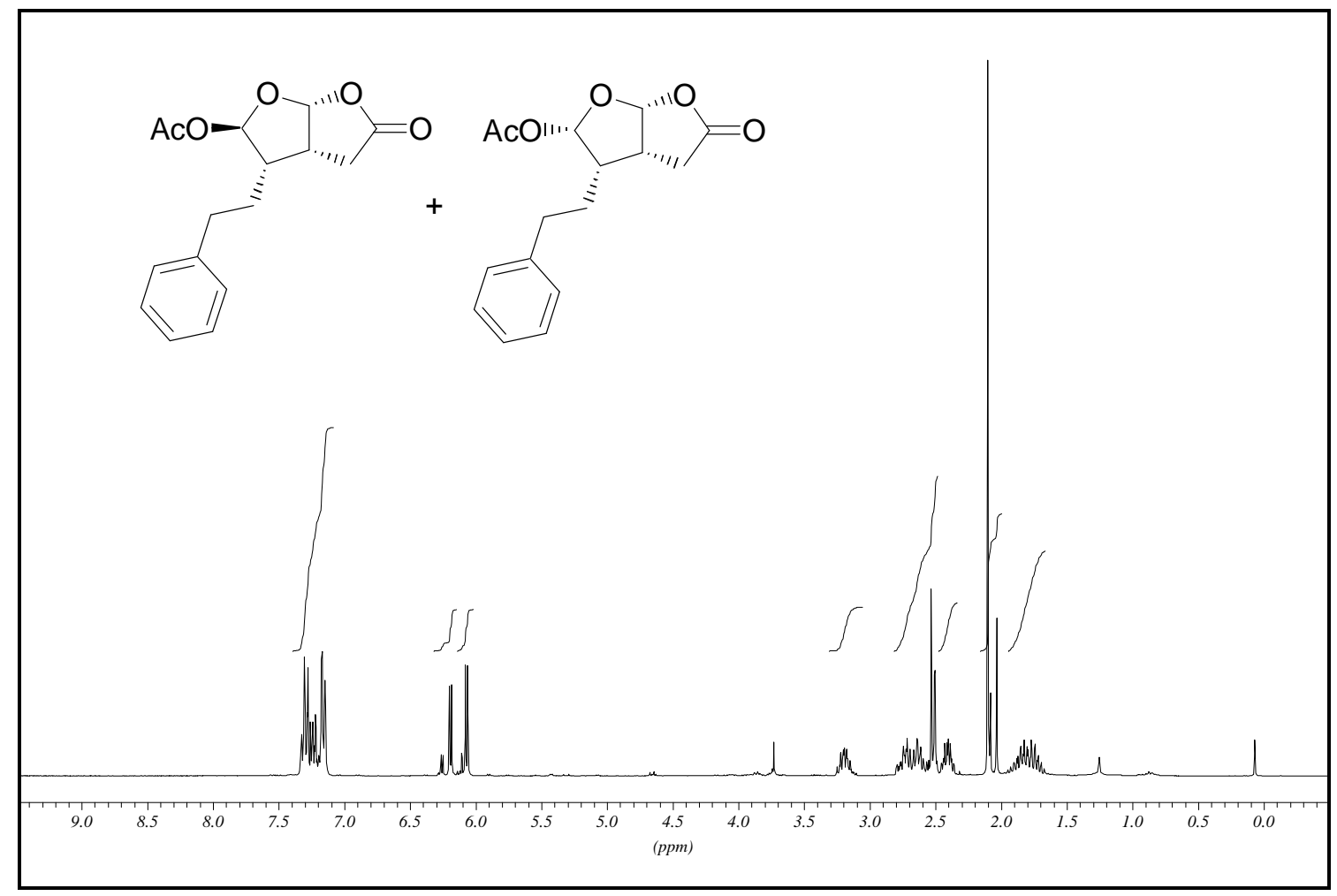

${ }^{13} \mathrm{C}$-NMR (75.5 MHz, $\mathrm{CDCl}_{3}$ )

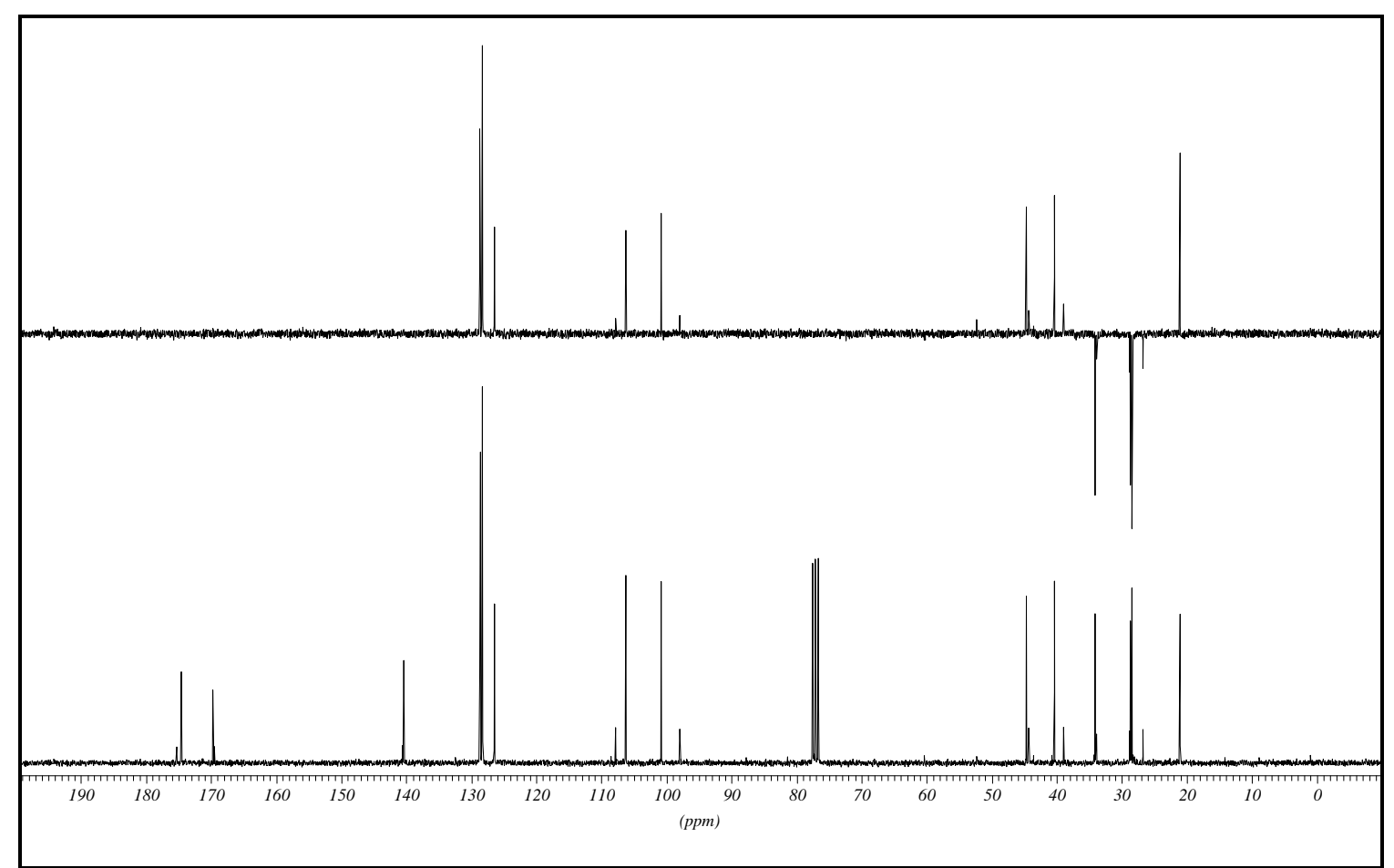

